\title{
HOMOLOGY OF $\mathrm{SL}_{2}$ OVER FUNCTION FIELDS I: PARABOLIC SUBCOMPLEXES
}

\author{
MATTHIAS WENDT
}

\begin{abstract}
The present paper studies the homology of the groups $\mathrm{SL}_{2}(k[C])$ and $\mathrm{GL}_{2}(k[C])$, where $C=\bar{C} \backslash\left\{P_{1}, \ldots, P_{s}\right\}$ is a smooth affine curve over an algebraically closed field $k$. It is well-known that these groups act on a product of trees and the quotients can be described in terms of certain equivalence classes of rank two vector bundles on the curve $\bar{C}$. There is a natural subcomplex consisting of cells with non-trivial isotropy group. The paper provides explicit formulas for the equivariant homology of this "parabolic subcomplex". These formulas also describe homology of $\mathrm{SL}_{2}(k[C])$ above degree $s$, generalizing a result of Suslin for the case $s=1$.
\end{abstract}

\section{Contents}

1. Introduction

2. Preliminaries: trees, group actions, vector bundles

3. Decomposable bundles and parabolic subcomplex

4. Local structure of parabolic subcomplexes

5. Global structure of parabolic subcomplexes

6. Equivariant homology of the parabolic subcomplex

Appendix A. Refined scissors congruence groups

References

\section{INTRODUCTION}

This paper is the first in a series of papers studying the homology of rank one linear groups over function rings and function fields of curves, mostly over algebraically closed fields. Of course, the groups $\mathrm{SL}_{2}$ and $\mathrm{GL}_{2}$, their structure, their homology and representation theory, have been subject to a lot of research in number-theoretic situations. However, surprisingly little information is available on the structure of $\mathrm{SL}_{2}$ in situations where the usual, more analytic, methods fail - for curves over infinite base fields.

That being said, the present paper still follows the standard path to computation of homology of linear groups over function rings of curves; the group $\mathrm{SL}_{2}(k[C])$ acts on a product of trees, the building $\mathfrak{X}_{C}$, and the isotropy spectral sequence associated to this action can be used to obtain information on group homology of $\mathrm{SL}_{2}(k[C])$. In general, understanding the structure of the quotient $\mathrm{SL}_{2}(k[C]) \backslash \mathfrak{X}_{C}$ is rather difficult, and this is one of the main reasons for the lack of group homology computations. There is, nevertheless, one part of group homology that is easier to understand: taking inspiration from the number-theoretic situation, we consider

Date: April 2014.

2010 Mathematics Subject Classification. 20G10, 20E42.

Key words and phrases. cohomology, linear groups, vector bundles.

This work has been partially supported by the Alexander-von-Humboldt-Stiftung. 
a subcomplex of the building, called parabolic subcomplex $\mathfrak{P}_{C}$, cf. Definition 3.6. consisting of cells with non-unipotent stabilizer. The equivariant homology of this subcomplex is a function field analogue of Farrell-Tate homology, and sits in a long exact sequence, cf. Proposition 3.8

$$
\cdots \rightarrow \mathrm{H}_{\bullet}^{\mathrm{SL}_{2}(k[C])}\left(\mathfrak{P}_{C}\right) \rightarrow \mathrm{H}_{\bullet}\left(\mathrm{SL}_{2}(k[C])\right) \rightarrow \mathrm{H}_{\bullet}^{\mathrm{SL}_{2}(k[C])}\left(\mathfrak{U}_{C}\right) \rightarrow \cdots,
$$

where $\mathrm{H}_{\bullet}^{\mathrm{SL}_{2}(k[C])}\left(\mathfrak{U}_{C}\right)$ is an analogue of cuspidal homology (or homology of the Steinberg module) in the number-theoretic situations. Moreover, the equivariant homology of the parabolic subcomplex can be computed very explicitly in terms of the homology of (normalizers of) maximal tori and refined scissors congruence groups $\mathcal{R P}_{\bullet}^{1}(k)$, cf. Section A. The following is the main result of the paper; it describes the equivariant homology of the parabolic subcomplex for $\mathrm{SL}_{2}(k[C])$ with $k$ an algebraically closed field. For the proofs, cf. Lemma 5.2, Proposition 6.1 and Proposition 6.3.

Theorem 1. Let $k$ be an algebraically closed field, let $\bar{C}$ be a smooth projective curve over $k$, let $P_{1}, \ldots, P_{s} \in \bar{C}$ be closed points, and set $C=\bar{C} \backslash\left\{P_{1}, \ldots, P_{s}\right\}$. Denoting by $\mathfrak{P}_{C}$ the parabolic subcomplex of the building $\mathfrak{X}_{C}$, cf. Definition 3.6. we have the following formulas for the $\mathrm{SL}_{2}(k[C])$-equivariant homology of $\mathfrak{P}_{C}$ :

(1) The connected components of the parabolic subcomplex $\mathfrak{P}_{C}$ are indexed by the quotient set $\mathcal{K}(C)=\operatorname{Pic}(C) / \iota$ of the Picard group of $C$ modulo the involution $\iota: \mathcal{L} \mapsto \mathcal{L}^{-1}$. The result is a direct sum decomposition:

$$
\mathrm{H}_{\bullet}^{\mathrm{SL}_{2}(k[C])}\left(\mathfrak{P}_{C}, \mathbb{Z}[1 / 2]\right) \cong \bigoplus_{[\mathcal{L}] \in \mathcal{K}(C)} \mathrm{H}_{\bullet}^{\mathrm{SL}_{2}(k[C])}\left(\mathfrak{P}_{C}(\mathcal{L}), \mathbb{Z}[1 / 2]\right)
$$

(2) If $[\mathcal{L}] \in \mathcal{K}(C)$ is such that $\left.\left.\mathcal{L}\right|_{C} ¥ \mathcal{L}\right|_{C} ^{-1}$, then the homology of the component $\mathfrak{P}_{C}(\mathcal{L})$ is the homology of the group $k[C]^{\times}$:

$$
\mathrm{H}_{\bullet}^{\mathrm{SL}_{2}(k[C])}\left(\mathfrak{P}_{C}(\mathcal{L}), \mathbb{Z}[1 / 2]\right) \cong \mathrm{H}_{\bullet}\left(k[C]^{\times}, \mathbb{Z}[1 / 2]\right) .
$$

(3) If $[\mathcal{L}] \in \mathcal{K}(C)$ is such that $\left.\left.\mathcal{L}\right|_{C} \cong \mathcal{L}\right|_{C} ^{-1}$, then there is a long exact sequence

$$
\cdots \rightarrow \mathcal{R} \mathcal{P}_{i+1}^{1}(k) \otimes_{\mathbb{Z}} \mathbb{Z}\left[1 / 2, k[C]^{\times} /\left(k[C]^{\times}\right)^{2}\right] \rightarrow \mathrm{H}_{i}(\tilde{\mathcal{S N}}, \mathbb{Z}[1 / 2]) \rightarrow
$$$$
\rightarrow \mathrm{H}_{i}^{\mathrm{SL}_{2}(k[C])}\left(\mathfrak{P}_{C}(\mathcal{L}), \mathbb{Z}[1 / 2]\right) \rightarrow \mathcal{R} \mathcal{P}_{i}^{1}(k) \otimes_{\mathbb{Z}} \mathbb{Z}\left[1 / 2, k[C]^{\times} /\left(k[C]^{\times}\right)^{2}\right] \rightarrow \cdots,
$$

where $\tilde{\mathcal{S N}}$ denotes the group of monomial matrices in $\mathrm{SL}_{2}(k[C])$ and $\mathcal{R} \mathcal{P}_{i}^{1}(k)$ are refined scissors congruence groups, cf. Section $A$.

There is a similar result for the equivariant homology of the parabolic subcomplex for the group $\mathrm{PGL}_{2}$, cf. Proposition 6.1 and Proposition 6.4.

The result is proved by explicitly computing the quotient $\mathrm{SL}_{2}(k[C]) \backslash \mathfrak{P}_{C}$ and then working out the isotropy spectral sequence in detail. The analysis of the spectral sequence is significantly simplified by working with $\mathbb{Z}[1 / 2]$-coefficients, and it is not clear what modifications would be necessary to get a 2 -integral result. The exact sequence appearing in Theorem 1, point(3), above is induced from the one connecting group homology of $\mathrm{SL}_{2}(k)$ to group homology of the normalizer of the maximal torus in $\mathrm{SL}_{2}(k)$ and refined scissors congruence groups $\mathcal{R} \mathcal{P}_{i}^{1}$. The latter exact sequence would appear to be well-known, cf. Dup01, Chapters 8, 15], but for the sake of completeness we discuss the definition of the groups $\mathcal{R} \mathcal{P}_{i}^{1}$ as well as a proof of the abovementioned exact sequence in Section $\mathrm{A}$.

It has been known for some time, and surfaced particularly in the recent work of Kevin Hutchinson [Hut11a, Hut11b], that the action of square classes $F^{\times} /\left(F^{\times}\right)^{2}$ is an extremely helpful tool in understanding group homology of $\mathrm{SL}_{2}(F)$. The result above exhibits a complete description as well as a geometric interpretation of the square-class action on the parabolic part of group homology. 
The above computations of the equivariant homology of the parabolic subcomplex also imply formulas for group homology of $\mathrm{SL}_{2}(k[C])$ above the dimension of the product of trees. The next result follows directly from a mod $\ell$-version of Theorem 1 and Proposition 3.8.

Corollary 1.1. Let $k$ be an algebraically closed field, let $\bar{C}$ be a smooth projective curve over $k$, and set $C=\bar{C} \backslash\left\{P_{1}, \ldots, P_{s}\right\}$. Let $\ell$ be an odd prime different from the characteristic of $k$. For $i>s$, there are isomorphisms

$$
\mathrm{H}_{i}^{\mathrm{SL}_{2}(k[C])}\left(\mathfrak{P}_{C}, \mathbb{Z} / \ell\right) \cong \mathrm{H}_{i}\left(\mathrm{SL}_{2}(k[C]), \mathbb{Z} / \ell\right) .
$$

In particular, we have a direct sum decomposition for mod $\ell$ group homology of $\mathrm{SL}_{2}(k[C])$ above homological degree $s$

$$
\begin{aligned}
& \mathrm{H}_{\bullet>s}\left(\mathrm{SL}_{2}(k[C]), \mathbb{Z} / \ell\right) \cong \bigoplus_{[\mathcal{L}] \in \mathcal{K}(C),\left.\mathcal{L}\right|_{C} \neq\left.\mathcal{L}\right|_{C} ^{-1}} \mathrm{H}_{\bullet>s}\left(k[C]^{\times}, \mathbb{Z} / \ell\right) \\
& \oplus \bigoplus_{\bullet} \mathrm{H}_{\bullet>s}^{\mathrm{SL}_{2}(k[C])}\left(\mathfrak{P}_{C}(\mathcal{L}), \mathbb{Z} / \ell\right)
\end{aligned}
$$

as well as an exact sequence describing the normalizer terms:

$$
\begin{gathered}
\cdots \rightarrow \mathcal{R} \mathcal{P}_{i+1}^{1}(k, \mathbb{Z} / \ell)\left[k(C)^{\times} /\left(k(C)^{\times}\right)^{2}\right] \rightarrow \mathrm{H}_{i}(\tilde{\mathcal{S N}}, \mathbb{Z} / \ell) \rightarrow \\
\mathrm{H}_{i}^{\mathrm{SL}_{2}(k[C])}\left(\mathfrak{P}_{C}(\mathcal{L}), \mathbb{Z} / \ell\right) \rightarrow \mathcal{R} \mathcal{P}_{i}^{1}(k, \mathbb{Z} / \ell)\left[k(C)^{\times} /\left(k(C)^{\times}\right)^{2}\right] \rightarrow \cdots
\end{gathered}
$$

Note that the above exact sequence is not simply the exact sequence of Theorem 1 tensored with finite coefficients, but using a $\bmod \ell$-version of the groups $\mathcal{R P}_{\bullet}^{1}(k)$, cf. Section A

The above theorem provides a generalization of a theorem of Suslin, cf. Knu01, Theorem 4.5.7]; for $s=1$ the above formula reduces to the one in loc.cit.:

$$
\begin{aligned}
\mathrm{H}_{\bullet>1}\left(\mathrm{PGL}_{2}(k[C]), \mathbb{Z} / \ell\right) & \cong \bigoplus_{\mathcal{L} \in \mathcal{K}(C), 2 \mathcal{L}=0} \mathrm{H}_{\bullet>1}\left(\mathrm{PGL}_{2}(k), \mathbb{Z} / \ell\right) \\
& \oplus \bigoplus_{\mathcal{L} \in \mathcal{K}(C), 2 \mathcal{L} \neq 0} \mathrm{H}_{\bullet>1}\left(k^{\times}, \mathbb{Z} / \ell\right) .
\end{aligned}
$$

Note that the finite coefficients are necessary: on the curve $\bar{C}$, there may exist bundles with unipotent automorphism group (like Atiyah's bundles $\mathcal{F}_{2}$ on an elliptic curve). The automorphism groups influence the homology in degrees above $s$, but are not accounted for in the parabolic subcomplex $\mathfrak{P}_{C}$. They do, however, not influence the $\bmod \ell$ homology above degree $s$ because the additive group $(k,+)$ is uniquely $\ell$-divisible.

The definition of the parabolic subcomplex is functorial in the curve, hence morphisms of curves induce morphisms on equivariant homology of the corresponding parabolic subcomplexes. The induced morphisms can be explicitly described and, for a function field $k(C)$, allow to define a "parabolic homology" of $S L_{2}(k(C))$ via the obvious limit process. In analogy to the notation for Farrell-Tate homology, we denote

$$
\widehat{\mathrm{H}}_{\bullet}\left(\mathrm{SL}_{2}(k(C)), \mathbb{Z} / \ell\right)=\underset{S \subseteq \bar{C}(k)}{\operatorname{colim}} \mathrm{H}_{\bullet}^{\mathrm{SL}_{2}(k[\bar{C} \backslash S])}(\mathfrak{P} \bar{C} \backslash S, \mathbb{Z} / \ell),
$$

where the index set of the colimit above is the set of finite sets of closed points of $\bar{C}$, ordered by inclusion. The second main result of the paper now provides a formula for the parabolic homology of $\mathrm{SL}_{2}(k(C))$ and establishes a rigidity result for it. It may not come as a surprise, but taking the limit to the algebraic closure of a function field, the parabolic homology has exactly the form predicted by the Friedlander-Milnor conjecture, cf. FM84. The result follows from Proposition 6.5 and the above Theorem 1 resp. its corollary, cf. Proposition 6.7. 
Theorem 2. Let $k$ be an algebraically closed field, let $C$ be a smooth curve over $k$ and let $\ell$ be an odd prime different from the characteristic of $k$. Then we have the following exact sequence

$$
\begin{aligned}
& \cdots \rightarrow \mathcal{R P}_{i+1}^{1}(k, \mathbb{Z} / \ell)\left[k(C)^{\times} /\left(k(C)^{\times}\right)^{2}\right] \rightarrow \mathrm{H}_{i}(\mathrm{~N}(k(C)), \mathbb{Z} / \ell) \rightarrow \\
& \rightarrow \widehat{\mathrm{H}}_{i}\left(\mathrm{SL}_{2}(k(C)), \mathbb{Z} / \ell\right) \rightarrow \mathcal{R} \mathcal{P}_{i}^{1}(k, \mathbb{Z} / \ell)\left[k(C)^{\times} /\left(k(C)^{\times}\right)^{2}\right] \rightarrow \cdots,
\end{aligned}
$$

where $\mathrm{N}(k(C))$ denotes the normalizer of a maximal torus in $\mathrm{SL}_{2}(k(C))$.

(1) All classes of $\widehat{\mathrm{H}}_{i}\left(\mathrm{SL}_{2}(k(C)), \mathbb{Z} / \ell\right)$ become constant over the quadratic closure of $k(C)$.

(2) Assume $\mathcal{R P}_{\bullet}^{1}(k)=0$, i.e., Friedlander's generalized isomorphism conjecture is true for $\mathrm{SL}_{2}$ over $k$. Then

$$
\begin{aligned}
\widehat{\mathrm{H}}_{\bullet}\left(\mathrm{SL}_{2}(\overline{k(C)}), \mathbb{Z} / \ell\right) & :=\underset{K / k(C) \text { finite }}{\text { colim }} \widehat{\mathrm{H}}_{\bullet}\left(\mathrm{SL}_{2}(K), \mathbb{Z} / \ell\right) \\
& \cong \mathrm{H}_{\bullet}(\mathrm{N}(\overline{k(C)}), \mathbb{Z} / \ell)
\end{aligned}
$$

has exactly the form predicted by Friedlander's isomorphism conjecture for $\mathrm{SL}_{2}$ over $\overline{k(C)}$. In particular, Friedlander's generalized isomorphism conjecture for $\mathrm{SL}_{2}$ over $\overline{k(C)}$ is equivalent to vanishing of

$$
\underset{C / k}{\operatorname{colim}} \mathrm{H}_{\bullet}^{\mathrm{SL}_{2}(k[C])}\left(\mathfrak{U}_{C}, \mathbb{Z} / \ell\right),
$$

where the colimit runs over all smooth affine curves over $k$.

The above result provides two reformulations of Friedlander's isomorphism conjecture, one as a divisibility result for "the limit of cuspidal homology", and one as a detection result of homology on the normalizer of the maximal torus. The second reformulation in turn is close to Knu01, Corollary 5.2.10]. In general, we see that the parabolic homology $\widehat{\mathrm{H}}_{\bullet}\left(\mathrm{SL}_{2}(k(C)), \mathbb{Z} / \ell\right)$ describes exactly the part of the homology of $\mathrm{SL}_{2}(k(C))$ which can be detected on the normalizer $\mathrm{N}(k(C))$ and some additional subgroups of $\mathrm{SL}_{2}(k(C))$ isomorphic to $\mathrm{SL}_{2}(k)$. Moreover, we see that the parabolic homology satisfies a much stronger rigidity than expected by Friedlander's isomorphism conjecture: classes in the parabolic homology become trivial already over quadratically closed fields. Hopefully, the above result helps shed new light on the homology of $\mathrm{SL}_{2}$ over algebraically closed fields.

Finally, we want to mention that related (and as it turns out structurally similar) computations in the number field case, i.e., computations of Farrell-Tate cohomology of $\mathrm{SL}_{2}\left(\mathcal{O}_{K, S}\right)$ with $\mathcal{O}_{K, S}$ a ring of $S$-integers, are being developed in joint work with Alexander D. Rahm.

1.1. Structure of the paper: We first recall preliminaries on trees and group actions in Section 2. The definition and basic properties of the parabolic subcomplex $\mathfrak{P}_{C}$ are given in Section 3. Then Section 4 works out the actions of stabilizers on links and the resulting local structure of the quotient of the parabolic subcomplex, leading to a global description of the structure of the quotient $\mathrm{SL}_{2}(k[C]) \backslash \mathfrak{P}_{C}$ in Section 5 . The structure of $\mathrm{SL}_{2}(k[C])$-equivariant homology of the parabolic subcomplex $\mathfrak{P}_{C}$ is determined in Section 6. The appendix Section $\mathrm{A}$ provides a recollection of basic facts on the refined scissors congruence groups $\mathcal{R P}_{\bullet}^{1}(k)$.

1.2. Acknowledgements: The investigations reported in the paper started during a stay at the De Brún center for computational algebra at NUI Galway in August 2012. I would like to thank Alexander D. Rahm for discussions about FarrellTate cohomology and his computations with Bianchi group [Rah13, Rah14, which shaped my understanding of the structure and possible usefulness of the parabolic part of group homology described in the paper. I would also like to thank Kevin 
Hutchinson for explanations on his computations of homology of $\mathrm{SL}_{2}$ and residue maps for refined Bloch groups in [Hut11a, Hut11b as well as inspiring discussions on various topics related to group homology.

\section{Preliminaries: trees, Group actions, Vector Bundles}

In this section, we fix the notation for the paper, and recall preliminaries on rank one linear groups and (products of) trees associated to them. We also discuss the well-known identification of the quotient of the building modulo the group action in terms of vector bundles on the curve.

We mostly follow the notation of [Ser80].

- $k$ denotes a commutative field,

- $\bar{C}$ an irreducible smooth projective curve over $k$,

- $C=\bar{C} \backslash\left\{P_{1}, \ldots, P_{s}\right\}$ a smooth affine curve over $k$, with $P_{1}, \ldots, P_{s}$ pairwise distinct but not necessarily $k$-rational points of respective degrees $d_{i}=$ $\left[k\left(P_{i}\right): k\right]$,

- $K=k(C)$ the function field of the curve $C$,

- $k[C]$ the ring of functions on the curve $C$,

- for a closed point $Q$ of $C, v_{Q}$ denotes the corresponding valuation, $\mathcal{O}_{Q}$ the valuation ring, $k(Q)$ its residue field, $\operatorname{deg} Q=[k(Q): k]$ its degree.

2.1. Recollection on buildings. We recall the definition and structure of the Bruhat-Tits tree associated to $\mathrm{SL}_{2}$ over a field $K$ with a valuation $v$. For details on Bruhat-Tits trees, cf. [Ser80, II.1.1], for the more general theory of buildings, cf. AB08. All the statements below are standard and can be found in one of these books.

Definition 2.1. Let $K$ be a field equipped with a discrete valuation $v$. We denote by $\mathcal{O}_{v}$ the corresponding valuation ring with maximal ideal $\mathfrak{m}_{v}$. We denote by $\pi_{v}$ a choice of uniformizer for $v$. Let $V=K^{2}$. A lattice $L$ in $V$ is a finitely generated $\mathcal{O}_{v}$-submodule of $V$ which generates $V$. Two lattices $L_{1}$ and $L_{2}$ are called equivalent if there exists $\lambda \in K^{\times}$such that $\lambda L_{1}=L_{2}$. We denote by $\Lambda=[L]$ the equivalence class of $L$. To a lattice class $\Lambda$, we can assign a type $v(\operatorname{det} \Lambda) \bmod 2 \in \mathbb{Z} / 2 \mathbb{Z}$.

The Bruhat-Tits tree associated to $(K, v)$ is the following simplicial complex: the 0-simplices are equivalence classes of lattices. Lattice classes $\Lambda_{0}$ and $\Lambda_{1}$ are connected by an edge if there exist representatives $L_{i}$ of $\Lambda_{i}$ such that $\pi_{v} L_{1} \subset L_{0} \subset$ $L_{1}$. The resulting simplicial complex is a tree, denoted by $\mathfrak{T}_{v}$.

There is an obvious action of $\mathrm{GL}_{2}\left(K_{v}\right)$ on $\mathfrak{T}_{v}$ by setting

$$
\mathrm{GL}_{2}\left(K_{v}\right) \times \mathfrak{T}_{v} \rightarrow \mathfrak{T}_{v}:(m, \Lambda) \mapsto m \Lambda
$$

Note that $\mathrm{GL}_{2}\left(K_{v}\right)$ acts transitively on the vertices of the Bruhat-Tits tree for $v$, and $\mathrm{SL}_{2}\left(K_{v}\right)$ acts transitively on the vertices of fixed type. The center acts trivially, i.e., the above actions on the building factor through actions of $\operatorname{PGL}_{2}\left(K_{v}\right)$ and $\mathrm{PSL}_{2}\left(K_{v}\right)$, respectively.

For each vertex $x=[L]$ with representative lattice $L$, there is a bijection between the link $\operatorname{Lk}(x)$ and the lattices $L^{\prime}$ with $\pi_{v} L \subset L^{\prime} \subset L$, hence with one-dimensional subspaces of the two-dimensional $k_{v}$-vector space $L / \pi_{v} L$. Thus, the elements of the link $\operatorname{Lk}(x)$ are in bijection with the $k_{v}$-points of the projective line $\mathbb{P}^{1}\left(k_{v}\right)$, where $k_{v}$ is the residue field of the valuation $v$ on $K$. In particular, the tree is homogeneous.

We can be more precise about the correspondence between the link and $\mathbb{P}^{1}\left(k_{v}\right)$ : it is induced by mapping the points $x \in \mathbb{A}^{1}\left(k_{v}\right)$ and $x=\infty$ to the lattice classes

$$
\left[L \cdot\left(\begin{array}{cc}
\pi_{v} & \widetilde{x} \\
0 & 1
\end{array}\right)\right] \text { and }\left[L \cdot\left(\begin{array}{cc}
\pi_{v}^{-1} & 0 \\
0 & 1
\end{array}\right)\right] \text {, }
$$


where $\tilde{x}$ is a lift of $x \in k_{v}$ to $\mathcal{O}_{v}$. This description of the correspondence of course depends on a choice of basis vector for the lines in $k_{v}^{2}$, a choice of lift to $L$ and a choice of completion to a basis of $L$.

Let $k$ be a field, let $\bar{C}$ be a smooth projective curve over $k$, and set $C=\bar{C}$ $\left\{P_{1}, \ldots, P_{s}\right\}$ for a non-empty finite set of pairwise distinct closed points $P_{1}, \ldots, P_{s}$ of $C$. Denote by $v_{1}, \ldots, v_{s}$ the corresponding valuations on the function field $K=$ $k(C)$, and denote by $\mathfrak{T}_{i}$ the Bruhat-Tits tree associated to the valuation $v_{i}$. Then the group $\mathrm{SL}_{2}(k[C])$ acts on $\mathfrak{X}_{C}:=\mathfrak{T}_{1} \times \cdots \times \mathfrak{T}_{s}$ via the embedding

$$
\mathrm{SL}_{2}(k[C]) \hookrightarrow \mathrm{SL}_{2}(k(C)) \hookrightarrow \mathrm{SL}_{2}\left(k(C)_{v_{1}}\right) \times \cdots \times \mathrm{SL}_{2}\left(k(C)_{v_{s}}\right) .
$$

The product $\mathfrak{X}_{C}=\mathfrak{T}_{1} \times \cdots \times \mathfrak{T}_{s}$ is the Bruhat-Tits building associated to $\mathrm{SL}_{2}$ and the smooth affine curve $C$. We view it as a cubical complex of dimension $s$ whose non-degenerate cubes are products of edges from the factors $\mathfrak{T}_{i}$.

To describe the local structure of the product, we consider the link of 0-simplices. Recall that for $X$ a cubical complex and $\sigma$ a 0 -cube of $X$, the link $\operatorname{Lk}_{X}(\sigma)$ of $\sigma$ in $X$ is the following simplicial complex: its 0 -simplices are the 0 -cubes of $X$ connected to $\sigma$ via a 1 -cube, and 0 -simplices $\sigma_{0}, \ldots, \sigma_{m}$ span an $m$-simplex if there exists an $(m+1)$-cube containing $\sigma, \sigma_{0}, \ldots, \sigma_{m}$. For a vertex $\left(x_{1}, \ldots, x_{s}\right)$ in $\mathfrak{T}_{1} \times \cdots \times \mathfrak{T}_{s}$, the link of $\left(x_{1}, \ldots, x_{s}\right)$ is then the following simplicial complex: its set of 0 -simplices is the disjoint union $\bigsqcup_{i=1}^{s} \operatorname{Lk}_{\mathfrak{T}_{i}}\left(x_{i}\right)$, and for each choice of index set $I \subseteq\{1, \ldots, s\}$ of cardinality $n$ and elements $\left\{y_{i} \in \operatorname{Lk}_{\mathfrak{T}_{i}}\left(x_{i}\right)\right\}_{i \in I}$, there is an $n$-simplex $\left(y_{1}, \ldots, y_{n}\right)$ corresponding to the $(n+1)$-cube spanned by $\left(x_{1}, \ldots, x_{s}\right)$ and the $y_{i}$. In particular, for $n=s=2$, the link of $\left(x_{1}, x_{2}\right)$ is the complete bipartite graph on the links of $x_{1}$ and $x_{2}$.

Again, we can be more precise about the lattices in the link: assume that the vertex $\left(x_{1}, \ldots, x_{s}\right)$ is represented by the lattice classes $\left(\left[L_{1}\right], \ldots,\left[L_{s}\right]\right)$. Then for each choice of index $i \in\{1, \ldots, s\}$ and element $\alpha_{i} \in \mathbb{P}^{1}\left(k\left(P_{i}\right)\right)$, the corresponding point in the link of $\left(x_{1}, \ldots, x_{s}\right)$ is given by $\left(\left[L_{1}\right], \ldots,\left[L_{i} \cdot M_{i}\right], \ldots,\left[L_{s}\right]\right)$, where $M_{i}$ is the corresponding matrix

$$
M_{i}=\left(\begin{array}{cc}
\pi_{i} & \widetilde{\alpha}_{i} \\
0 & 1
\end{array}\right) \operatorname{resp.}\left(\begin{array}{cc}
\pi_{i}^{-1} & 0 \\
0 & 1
\end{array}\right) .
$$

2.2. Buildings and vector bundles. As discussed, for a smooth affine curve $C=\bar{C} \backslash\left\{P_{1}, \ldots, P_{s}\right\}$, the groups $\mathrm{GL}_{2}(k[C])$ and $\mathrm{SL}_{2}(k[C])$ act on the Bruhat-Tits building $\mathfrak{X}_{C}$. It is well-known that the quotient can be described in terms of vector bundles on the curve $\bar{C}$, cf. [Ser80, Propositions II.2.4, II.2.5], [Stu76] and Stu80]. We recall the necessary steps.

Definition 2.2. Let $\left(L_{1}, \ldots, L_{s}\right)$ be a tuple, in which $L_{i}$ is an $\mathcal{O}_{P_{i}}$-lattice in $V=K^{2}$. We associate to this point in $\mathfrak{X}_{C}$ the unique coherent subsheaf $\mathcal{E}=$ $\mathcal{E}\left(L_{1}, \ldots, L_{s}\right)$ of the constant sheaf $V$ given by taking the stalk of $\mathcal{E}$ at a point $Q \in \bar{C}$ to be $L_{i}$ if $Q=P_{i}$ and $\mathcal{O}_{Q}^{2}$ otherwise.

Two vector bundles $\mathcal{E}_{1}$ and $\mathcal{E}_{2}$ are called equivalent rel $\partial C$ if there exist integers $m_{1}, \ldots, m_{s}$ such that

$$
\mathcal{E}_{1} \otimes \mathcal{O}_{\bar{C}}\left(-P_{1}\right)^{m_{1}} \otimes \cdots \otimes \mathcal{O}_{\bar{C}}\left(-P_{s}\right)^{m_{s}} \cong \mathcal{E}_{2},
$$

where $\mathcal{O}_{\bar{C}}(-P)$ is the ideal sheaf of functions vanishing at $P$.

It is easy to see that $\mathcal{E}\left(L_{1}, \ldots, L_{s}\right)$ is the sheaf of sections of a rank two vector bundle over $\bar{C}$ whose restriction to $C$ is trivial. In the following, there will be no notational distinction between the sheaf of sections and the vector bundle. It also follows easily that for scalars $\lambda_{1}, \ldots, \lambda_{s} \in K^{\times}$, we have

$$
\mathcal{E}\left(L_{1}, \ldots, L_{s}\right) \otimes \mathcal{O}_{\bar{C}}\left(-P_{1}\right)^{v_{1}\left(\lambda_{1}\right)} \otimes \cdots \otimes \mathcal{O}_{\bar{C}}\left(-P_{s}\right)^{v_{s}\left(\lambda_{s}\right)} \cong \mathcal{E}\left(\lambda_{1} L_{1}, \ldots, \lambda_{s} L_{s}\right) .
$$


From these remarks, we have the following identification of the quotient of the building in terms of vector bundles.

Proposition 2.3. The two assignments

$$
\left(L_{1}, \ldots, L_{s}\right) \mapsto\left(\left[L_{1}\right], \ldots,\left[L_{s}\right]\right) \in \mathfrak{X}_{C}, \text { and }\left(L_{1}, \ldots, L_{s}\right) \mapsto \mathcal{E}\left(L_{1}, \ldots, L_{s}\right)
$$

induce a bijection between

(1) the 0-cells of the quotient $\mathrm{SL}_{2}(k[C]) \backslash \mathfrak{X}_{C}$, and

(2) equivalence $($ rel $\partial C$ ) classes of pairs $(\mathcal{E}, f)$ where $\mathcal{E}$ is a rank two vector bundle on $\bar{C}$ whose restriction to $C$ is trivial, and

$$
f: \operatorname{det} \mathcal{E} \rightarrow \mathcal{O}_{\bar{C}}\left(-P_{1}\right)^{m_{1}} \otimes \cdots \otimes \mathcal{O}_{\bar{C}}\left(-P_{s}\right)^{m_{s}}
$$

is a fixed isomorphism for suitable $m_{i}$.

This bijection further induces (by forgetting the fixed determinant) a bijection between

(1) the 0-cells of the quotient $\mathrm{GL}_{2}(k[C]) \backslash \mathfrak{X}_{C}$, and

(2) equivalence (rel $\partial C$ ) classes of rank two vector bundles $\mathcal{E}$ on $\bar{C}$ whose restriction to $C$ is trivial.

Remark 2.4. The vector bundle classification is usually stated in different form: the set of isomorphism classes of rank two vector bundles on $\bar{C}$ whose restriction to $C$ is trivial is parametrized by the double quotient

$$
\mathrm{GL}_{2}(k[C]) \backslash\left(\prod_{i=1}^{s} \mathrm{GL}_{2}\left(k(C)_{v_{i}}\right)\right) /\left(\prod_{i=1}^{s} \mathrm{GL}_{2}\left(\mathcal{O}_{v_{i}}\right)\right) .
$$

In the building, we additionally divide out the centers of the groups $\mathrm{GL}_{2}\left(k(C)_{v_{i}}\right)$ which leads to the additional equivalence (rel $\partial C$ ) on vector bundles. There is no difference between using $\mathrm{GL}_{2}(k(C))$ or $\mathrm{GL}_{2}\left(k(C)_{v_{i}}\right)$ in the above, the right cosets of the corresponding maximal compact group are the same.

Under the correspondence described above, the link of a point $x=\left(\left[L_{1}\right], \ldots,\left[L_{s}\right]\right)$ corresponding to the vector bundle $\mathcal{E}=\mathcal{E}\left(L_{1}, \ldots, L_{s}\right)$ is given by the vector bundles $\mathcal{E}^{\prime}$ which arise from $\mathcal{E}$ by elementary transformations: there is an edge between the vector bundles $\mathcal{E}$ and $\mathcal{E}^{\prime}$ if there is an embedding $\mathcal{E}^{\prime} \hookrightarrow \mathcal{E}$ of $\mathcal{E}^{\prime}$ as a subsheaf of $\mathcal{E}$ and the quotient $\mathcal{E} / \mathcal{E}^{\prime}$ is a torsion $\mathcal{O}_{\bar{C}}$-module of length one, concentrated in one of the points $P_{1}, \ldots, P_{s}$. Similarly, the $n$-simplices in the link of $x$ are given by the choice of $n+1$ such elementary transformations at $n+1$ pairwise distinct points in $\left\{P_{1}, \ldots, P_{s}\right\}$.

The correspondence between vertices in the quotient of the building and equivalence classes of vector bundles also allows the identification of stabilizers of vertices with automorphism groups of vector bundles.

Proposition 2.5. Let $x=\left(\left[L_{1}\right], \ldots,\left[L_{s}\right]\right)$ be a point in the building $\mathfrak{X}_{C}$ with associated vector bundle $\mathcal{E}=\mathcal{E}\left(L_{1}, \ldots, L_{s}\right)$. Then we have the following description of stabilizers, where $\operatorname{Stab}(x ; G)$ denotes the stabilizer of the vertex $x$ in the group $G$ :

(1) $\operatorname{Stab}\left(x ; \mathrm{GL}_{2}(k[C])\right) \cong \operatorname{Aut}(\mathcal{E}) \times_{k^{\times}} k[C]^{\times}$, where $k^{\times} \rightarrow \operatorname{Aut}(\mathcal{E})$ denotes the embedding of the homotheties,

(2) $\operatorname{Stab}\left(x ; \operatorname{PGL}_{2}(k[C])\right) \cong \operatorname{Aut}(\mathcal{E}) / k^{\times}$

(3) $\operatorname{Stab}\left(x ; \mathrm{SL}_{2}(k[C])\right) \cong \operatorname{Aut}(\mathcal{E}, f)$, where $\operatorname{Aut}(\mathcal{E}, f)$ denotes those automorphisms commuting with the map $f: \operatorname{det} \mathcal{E} \rightarrow \mathcal{O}_{\bar{C}}\left(-P_{1}\right)^{m_{1}} \otimes \cdots \otimes \mathcal{O}_{\bar{C}}\left(-P_{s}\right)^{m_{s}}$ from Proposition 2.3.

The stabilizers of a cube in any of the above groups are given by the intersection of the respective stabilizers of its vertices. The intersections are taken in the groups $\mathrm{GL}_{2}(K), \mathrm{PGL}_{2}(K)$ and $\mathrm{SL}_{2}(K)$, respectively. 
Proof. (1) We first describe a homomorphism

$$
\operatorname{Aut}(\mathcal{E}) \times_{k} \times k[C]^{\times} \rightarrow \operatorname{Stab}\left(x ; \mathrm{GL}_{2}(k[C])\right) .
$$

Note that the bundle $\mathcal{E}$ comes with an explicit embedding into $K^{2}$, in particular the restriction of $\mathcal{E}$ to $C$ comes with a given trivialization. An automorphism $\phi \in \operatorname{Aut}(\mathcal{E})$ can be restricted to a $k[C]$-linear automorphism of $\left.\mathcal{E}\right|_{C}$, and the given trivialization allows to write this as an element in $\mathrm{GL}_{2}(k[C])$. This produces a homomorphism $\operatorname{Aut}(\mathcal{E}) \rightarrow \mathrm{GL}_{2}(k[C])$ which lands inside $\operatorname{Stab}\left(x ; \mathrm{GL}_{2}(k[C])\right)$ because the automorphism preserves all the lattices. There is also a homomorphism $k[C]^{\times} \rightarrow \operatorname{Stab}\left(x ; \mathrm{GL}_{2}(k[C])\right)$ given by embedding $k[C]^{\times}$into the center of $\mathrm{GL}_{2}(k[C])$ by

$$
u \in k[C]^{\times} \mapsto\left(\begin{array}{cc}
u & 0 \\
0 & u
\end{array}\right) .
$$

This also preserves the lattice classes $\left[L_{i}\right]$, hence stabilizes $x$. We can embed $k^{\times} \hookrightarrow \operatorname{Aut}(\mathcal{E})$ as the homotheties, and $k^{\times} \hookrightarrow k[C]^{\times}$as constants. On $k^{\times}$, the homomorphisms given above agree, hence we obtain the required homomorphism. This homomorphism is obviously injective.

By definition, the elements of $\operatorname{Stab}\left(x ; \mathrm{GL}_{2}(k[C])\right)$ preserve the lattice classes $\left[L_{i}\right]$. Moreover, any element of $\mathrm{GL}_{2}(k[C])$ preserves the standard lattice $\mathcal{O}_{Q}^{2}$ for $Q \notin\left\{P_{1}, \ldots, P_{s}\right\}$. Therefore, any element of the stabilizer which preserves the lattices $L_{i}$ (as opposed to just the lattice classes) is in fact an automorphism of the vector bundle $\mathcal{E}$. Now any element of the stabilizer can be factored as an element which preserves the lattices $L_{i}$ and some central element, hence the homomorphism is surjective.

(2) is obtained by dividing out the center on both sides. On the automorphism side, this is exactly dividing out the factor $k[C]^{\times}$. On the stabilizer side, this is precisely the passage from $\mathrm{GL}_{2}$ to $\mathrm{PGL}_{2}$.

(3) is obtained by restricting to determinant 1 .

Finally, the building is a $\mathrm{CAT}(0)$-space, and $\mathrm{GL}_{2}(k[C])$ acts via isometries. Therefore, if an element stabilizes vertices, it also stabilizes the cube they span. Stabilizers of cubes can then be computed by intersection of stabilizers of vertices inside the respective group.

2.3. Recollection on Jacobians of curves. We recall the Nagata exact sequence which describes units and Picard groups of smooth affine curves. This will be needed for the classification of (geometrically) split rank two bundles later on.

Lemma 2.6. Let $\bar{C}$ be a smooth projective curve over a field $k$, let $P_{1}, \ldots, P_{s}$ be closed points of degrees $d_{i}=\left[k\left(P_{i}\right): k\right]$, and denote $C=\bar{C} \backslash\left\{P_{1}, \ldots, P_{s}\right\}$. Then there is an exact sequence

$$
0 \rightarrow \mathcal{O}(\bar{C})^{\times}=k^{\times} \rightarrow \mathcal{O}(C)^{\times} \rightarrow \bigoplus_{i=1}^{s} \mathbb{Z} \stackrel{\phi}{\rightarrow} \operatorname{Pic}(\bar{C}) \rightarrow \operatorname{Pic}(C) \rightarrow 0
$$

In particular:

(1) There is an exact sequence

$$
\begin{aligned}
& \qquad 0 \rightarrow \operatorname{Pic}^{0}(C) \rightarrow \operatorname{Pic}(C) \rightarrow \mathbb{Z} / \operatorname{gcd}\left(d_{1}, \ldots, d_{s}\right) \mathbb{Z} \rightarrow 0, \\
& \text { where } \operatorname{Pic}^{0}(C)=\operatorname{Pic}^{0}(\bar{C}) /\left(\operatorname{Im} \phi \cap \operatorname{Pic}^{0}(\bar{C})\right) . \\
& \text { We have } \\
& \qquad k[C]^{\times} \cong k^{\times} \oplus \mathbb{Z}^{\operatorname{dim} \operatorname{ker} \phi} .
\end{aligned}
$$

(2) We have

Proof. The exact sequence is a special case of a localization sequence in K-theory or Chow groups. A more elementary proof of the exact sequence may be found e.g. in [Ros73, Proposition 1]. 
The morphism $\phi: \mathbb{Z}^{s} \rightarrow \operatorname{Pic}(\bar{C})$ sends the basis vector $e_{i}$ to the line bundle $\mathcal{O}_{\bar{C}}\left(-P_{i}\right)$, cf. [Har77, Proposition II.6.5]. Moreover, the degree map provides the standard exact sequence

$$
0 \rightarrow \operatorname{Pic}^{0}(\bar{C}) \rightarrow \operatorname{Pic}(\bar{C}) \rightarrow \mathbb{Z} \rightarrow 0 .
$$

The composition $\operatorname{deg} \circ \phi: \mathbb{Z}^{s} \rightarrow \operatorname{Pic}(\bar{C}) \stackrel{\operatorname{deg}}{\longrightarrow} \mathbb{Z}$ maps $e_{i}$ to $\operatorname{deg}\left(P_{i}\right)$, so its image is generated by $\operatorname{gcd}\left(d_{1}, \ldots, d_{s}\right)$. Thus $\operatorname{ker}(\operatorname{deg} \circ \phi) \cong \mathbb{Z}^{s-1}$ consists exactly of the degree zero divisors, and the map $\operatorname{ker} \phi \rightarrow \operatorname{Pic}(\bar{C})$ factors through $\mathbb{Z}^{s-1} \rightarrow \operatorname{Pic}^{0}(\bar{C})$. The rank of the latter map varies, according to possible rational equivalence relations between the points $P_{1}, \ldots, P_{s}$. This proves (1). The splitting and rank computation in (2) is obvious.

\section{Decomposable BUndles and parabolic SUbCOMPlex}

In this section, we use the identification of Proposition 2.5 to discuss the structure of stabilizer subgroups of vertices in $\mathfrak{X}_{C}$. Recalling some basics about automorphism groups of rank two vector bundles, we see that the existence of a non-trivial non-unipotent automorphism implies that the vector bundle is geometrically split. Based on this observation, we consider the subcomplex of the quotient which consists exactly of the cells which correspond to geometrically decomposable bundles. The equivariant cohomology of this complex is a version of Farrell-Tate cohomology, in fact agrees with it for finite base fields (and finite coefficients away from the characteristic).

3.1. Automorphisms and decomposability of vector bundles. We first recall the structure of automorphism groups of rank two vector bundles. These results in particular imply that decomposability of a rank two vector bundle can be seen from the structure of the automorphism group.

Proposition 3.1. Let $k$ be a field, let $\bar{C}$ be a smooth projective curve over $k$, and let $\mathcal{E}$ be a rank two vector bundle over $\bar{C}$.

(1) If $\mathcal{E}$ is geometrically indecomposable, then we have $\operatorname{End}\left(\mathcal{E}_{\bar{k}}\right) \cong \bar{k} \oplus \mathcal{N} i$. Every automorphism is a product of a central element and a unipotent element.

(2) If $\mathcal{E}$ is geometrically decomposable, then there exists a separable extension $L / k$ with $[L: k]=2$ such that $\mathcal{E}_{L} \cong \mathcal{L} \oplus \mathcal{L}^{\prime}$ with $\mathcal{L}$ and $\mathcal{L}^{\prime}$ line bundles over $\bar{C} \times_{k}$ L. There are three possibilities:

(a) If $\mathcal{L} \cong \mathcal{L}^{\prime}$, then both $\mathcal{L}$ and $\mathcal{L}^{\prime}$ as well as the splitting are defined over $k$. We have $\operatorname{End}(\mathcal{E}) \cong \mathrm{M}_{2}(k)$ and $\operatorname{Aut}(\mathcal{E}) \cong \mathrm{GL}_{2}(k)$.

(b) If $\mathcal{L} \neq \mathcal{L}^{\prime}$ and the splitting is defined over $k$, then we have (assuming $\left.\operatorname{deg} \mathcal{L} \geq \operatorname{deg} \mathcal{L}^{\prime}\right)$

$\operatorname{Aut}(\mathcal{E}) \cong\left\{\left(\begin{array}{ll}a & c \\ 0 & b\end{array}\right), \mid a, b \in k, a b \neq 0, c \in \mathrm{H}^{0}\left(\bar{C}, \mathcal{L} \otimes\left(\mathcal{L}^{\prime}\right)^{-1}\right)\right\}$.

(c) If $\mathcal{L} \neq \mathcal{L}^{\prime}$ and the splitting is not defined over $k$, then the automorphism group of $\mathcal{E}$ is the Weil restriction of a torus:

$$
\operatorname{Aut}(\mathcal{E}) \cong \mathrm{R}_{L / k}^{1}\left(\mathbb{G}_{m}\right) \text {. }
$$

Proof. (1) is due to Atiyah. In a complex analytic setting, it can be found as Proposition 15, its reformulation and Proposition 16 in Ati57. The algebraic result is proved similarly using Ati56].

(2) can be found in [Ser80, p. 101].

Corollary 3.2. A rank two vector bundle is geometrically split if and only if there exists a non-central non-unipotent automorphism. 
Remark 3.3. A similar pattern appears for the finite subgroups containing odd order elements in arithmetic groups $\mathrm{SL}_{2}\left(\mathcal{O}_{K, S}\right)$ : case (a) is a dihedral group, case (b) is a diagonalizable finite cyclic group, and case (c) a non-diagonalizable finite cyclic group.

3.2. Parabolic subcomplex and an exact sequence. In this paper, we are interested in the subcomplex of $\mathfrak{X}_{C}$ containing the decomposable bundles. More precisely, we consider the subcomplex of $\mathfrak{X}_{C}$ containing exactly the cells which have a non-central non-unipotent element in their stabilizer. This is justified by the fact that the unipotent radicals of stabilizers will not be visible in homology with finite coefficients away from the characteristic.

Lemma 3.4. Let $k$ be a field, let $\bar{C}$ be a smooth projective curve over $k$, and denote $C=\bar{C} \backslash\left\{P_{1}, \ldots, P_{s}\right\}$. The subset of the building $\mathfrak{X}_{C}$ consisting of the cells with non-unipotent stabilizer is in fact a subcomplex. The action of $\mathrm{GL}_{2}(k[C])$ resp. $\mathrm{SL}_{2}(k[C])$ on the building restricts to an action on this subcomplex.

Proof. As mentioned before, the stabilizers of $n$-cubes are the intersections of the stabilizers of their vertices. In particular, if an $n$-cube has non-unipotent stabilizer, then so do all its faces.

If two cubes are conjugate by the $\mathrm{GL}_{2}(k[C])$ - or the $\mathrm{SL}_{2}(k[C])$-action, then so are their stabilizers.

Remark 3.5. Note that this subcomplex is not necessarily a full subcomplex. Even if all the vertices of a cube have non-unipotent stabilizers, the cube does not necessarily have a non-unipotent stabilizer. It frequently happens that the automorphism groups of the vertices of a cube have trivial intersection inside $\mathrm{PGL}_{2}(K)$.

Definition 3.6. - The subcomplex of the building consisting of cells with non-unipotent stabilizer in $\mathrm{PGL}_{2}(k[C])$ is denoted by $\mathfrak{P}_{C}$ and is called the parabolic subcomplex.

- The quotient of the building $\mathfrak{X}_{C}$ modulo the subcomplex $\mathfrak{P}_{C}$ is denoted by $\mathfrak{U}_{C}$ and is called the unknown quotient.

Remark 3.7. The terminology "parabolic subcomplex" is supposed to underline that the stabilizers in the subcomplex are strongly related to parabolic (or better parahoric) subgroups of $\mathrm{GL}_{2}(K)$ or $\mathrm{SL}_{2}(K)$.

The terminology "unknown quotient" is supposed to underline our complete lack of knowledge of its structure, homology etc. There are strong links to cuspidal phenomena in number theory: the quotient $\mathfrak{U}_{C}$ contains the information away from infinity. In the case of finite base fields, the unknown quotients contains most of the compactly supported cohomology. However, "cuspidal quotient" seems inappropriate terminology, overloaded as the term cuspidal is in number theory and representation theory.

Exploration of the structure of the great unknown will be the subject of further papers in the series.

It now follows from the definition that we have an exact sequence of chain complexes.

Proposition 3.8. Let $k$ be a field, let $\bar{C}$ be a smooth projective curve over $k$, and denote $C=\bar{C} \backslash\left\{P_{1}, \ldots, P_{s}\right\}$.

(1) Denote by $\Gamma$ one of the linear groups $(\mathrm{P}) \mathrm{GL}_{2}(k[C])$ or $(\mathrm{P}) \mathrm{SL}_{2}(k[C])$. There is an exact sequence of $\Gamma$-chain complexes

$$
0 \rightarrow \mathrm{C} \bullet\left(\mathfrak{P}_{C}\right) \rightarrow \mathrm{C} \bullet\left(\mathfrak{X}_{C}\right) \rightarrow \mathrm{C}_{\bullet}\left(\mathfrak{U}_{C}\right) \rightarrow 0 .
$$


In particular, there is a long exact sequence of $\Gamma$-equivariant homology groups:

$$
\cdots \rightarrow \mathrm{H}_{\bullet+1}^{\Gamma}\left(\mathrm{C}_{\bullet}\left(\mathfrak{U}_{C}\right)\right) \rightarrow \mathrm{H}_{\bullet}^{\Gamma}\left(\mathrm{C}_{\bullet}\left(\mathfrak{P}_{C}\right)\right) \rightarrow \mathrm{H}_{\bullet}^{\Gamma}\left(\mathrm{C}_{\bullet}\left(\mathfrak{X}_{C}\right)\right) \rightarrow \mathrm{H}_{\bullet}^{\Gamma}\left(\mathrm{C}_{\bullet}\left(\mathfrak{U}_{C}\right)\right) \rightarrow \cdots
$$

Since $\mathfrak{X}_{C}$ is contractible, we also have $\mathrm{H}_{\bullet}^{\Gamma}\left(\mathrm{C}_{\bullet}\left(\mathfrak{X}_{C}\right)\right) \cong \mathrm{H}_{\bullet}(\Gamma)$.

(2) Assume now that $\Gamma$ is one of groups $\mathrm{PGL}_{2}(k[C])$ or $(\mathrm{P}) \mathrm{SL}_{2}(k[C])$, and let $\ell$ be an odd prime different from the characteristic of $k$. For $i>s$, we have $\mathrm{H}_{i}^{\Gamma}\left(\mathrm{C} \bullet\left(\mathfrak{U}_{C}\right), \mathbb{Z} / \ell\right)=0$. In particular, we have isomorphisms

$$
\mathrm{H}_{i}^{\Gamma}\left(\mathrm{C} \cdot\left(\mathfrak{P}_{C}\right)\right) \cong \mathrm{H}_{i}(\Gamma, \mathbb{Z} / \ell) .
$$

Proof. (1) is barely more than the definition of $\mathfrak{P}_{C}$ and $\mathfrak{U}_{C}$.

(2) By definition, the cells in $\mathfrak{U}_{C}$ have unipotent stabilizer in $\mathrm{PGL}_{2}(k[C])$ and $\operatorname{PSL}_{2}(k[C])$, hence they are uniquely $\ell$-divisible groups by assumption. The same holds for the stabilizers in $\mathrm{SL}_{2}(k[C])$ since the center has order 2. In particular, the isotropy spectral sequence computing $\mathrm{H}_{\bullet}^{\Gamma}\left(\mathrm{C}_{\bullet}\left(\mathfrak{U}_{C}\right), \mathbb{Z} / \ell\right)$ is concentrated in the line $q=0$ since only the groups $\mathrm{H}_{0}\left(\Gamma_{\sigma}, \mathbb{Z} / \ell_{\sigma}\right)$ are non-trivial. The spectral sequence therefore degenerates at the $E^{2}$-term and converges to

$$
\mathrm{H}_{\bullet}^{\Gamma}\left(\mathrm{C}_{\bullet}\left(\mathfrak{U}_{C}\right), \mathbb{Z} / \ell\right) \cong \mathrm{H}_{\bullet}\left(\mathrm{C} \bullet\left(\Gamma \backslash \mathfrak{U}_{C}\right), \mathbb{Z} / \ell\right) .
$$

The quotient only has cells in dimension $\leq s$, since the building only has dimension $s$. This proves the claim.

Remark 3.9. The exact sequence closely resembles the dual of the long exact sequence relating group cohomology to Farrell-Tate cohomology and the homology of the Steinberg module for groups of finite virtual cohomological dimension, cf. Bro94:

$$
\cdots \rightarrow \widehat{H}^{\bullet-1}(\Gamma) \rightarrow H_{n-\bullet}\left(\Gamma, \mathrm{St}^{\Gamma}\right) \rightarrow H^{\bullet}(\Gamma) \rightarrow \widehat{H}^{\bullet}(\Gamma) \rightarrow \cdots
$$

For finite base fields $k$, the groups $\mathrm{GL}_{2}(k[C])$ and $\mathrm{SL}_{2}(k[C])$ have finite virtual $\ell$ cohomological dimension if $\ell \neq$ char $k$ and the long exact sequence of Proposition 3.8 is the (dual of the) one for Farrell-Tate cohomology. For infinite base fields, the groups $\mathrm{GL}_{2}(k[C])$ and $\mathrm{SL}_{2}(k[C])$ do no longer have finite virtual cohomological dimension. Nevertheless, we can see the homology of the parabolic subcomplex as a replacement of Farrell-Tate homology in this setting.

Definition 3.10. Let $k$ be a field, let $\bar{C}$ be a smooth projective curve over $k$, and denote $C=\bar{C} \backslash\left\{P_{1}, \ldots, P_{s}\right\}$. Denote by $\Gamma$ one of the linear groups $(\mathrm{P}) \mathrm{GL}_{2}(k[C])$ or $(\mathrm{P}) \mathrm{SL}_{2}(k[C])$. The $\Gamma$-equivariant homology of the parabolic subcomplex $\mathfrak{P}_{C}$ is called the parabolic homology of $\Gamma$.

Remark 3.11. There is a version of Tate cohomology for arbitrary groups, due to Mislin [Mis94], which agrees with Farrell-Tate cohomology for groups of finite virtual cohomological dimension. It is not clear if Mislin's version of Tate cohomology agrees with the equivariant cohomology of the parabolic subcomplex above. The difficulty is mainly in the different definitions: while Mislin's version of Tate cohomology is defined via killing projectives in the derived category of $\mathbb{Z}[\Gamma]-$ modules, the parabolic subcomplex has only a very concrete geometric definition.

A first step in comparing the two cohomologies would be to compute the parabolic homology of a projective $\mathbb{Z}[\Gamma]$-module which is possible using the methods of the present paper. It could probably be shown that a projective $\mathbb{Z}\left[\mathrm{SL}_{2}(k[C])\right]$-module whose restriction to the constant group rings $\mathbb{Z}\left[\mathrm{SL}_{2}(k)\right]$ is injective, has trivial parabolic homology. This would at least establish a morphism from a "relative" MislinTate cohomology to parabolic cohomology. 
3.3. Functoriality. The parabolic subcomplex is also functorial with respect to morphisms of curves:

Proposition 3.12. Let $f: \bar{D} \rightarrow \bar{C}$ be a finite morphism of smooth projective curves over $k$, let $P_{1}, \ldots, P_{s}$ be points on $\bar{C}$, and let $Q_{1}, \ldots, Q_{t}$ be points on $\bar{D}$ not in the preimage of the $P_{i}$. Set $C=\bar{C} \backslash\left\{P_{1}, \ldots, P_{s}\right\}$ and $D=\bar{D} \backslash\left(\left\{f^{-1}\left(\left\{P_{1}, \ldots, P_{s}\right\}\right) \cup\right.\right.$ $\left.\left\{Q_{1}, \ldots, Q_{t}\right\}\right)$.

(After possible subdivision of $\mathfrak{X}_{C}$ ) there is a natural morphism $f^{*}: \mathfrak{X}_{C} \rightarrow \mathfrak{X}_{D}$ which induces a morphism $f^{*}: \mathfrak{P}_{C} \rightarrow \mathfrak{P}_{D}$ of parabolic subcomplexes. The morphism $f^{*}: \mathfrak{P}_{C} \rightarrow \mathfrak{P}_{D}$ is equivariant with respect to the natural group homomorphism $\mathrm{GL}_{2}(k[C]) \rightarrow \mathrm{GL}_{2}(k[D])$. This assignment is functorial.

There are induced morphisms $f^{*}: \mathrm{GL}_{2}(k[C]) \backslash \mathfrak{X}_{C} \rightarrow \mathrm{GL}_{2}(k[D]) \backslash \mathfrak{X}_{D}$ (and similarly for $\mathrm{SL}_{2}$ in place of $\mathrm{GL}_{2}$ resp. $\mathfrak{P}$ in place of $\left.\mathfrak{X}\right)$. In terms of the vector bundle interpretation of the quotient, these morphisms are identified with pullback of vector bundles.

Proof. The morphism of curves induces a morphism of function fields $f^{*}: k(C) \rightarrow$ $k(D)$. This is compatible with the valuations and hence induces a morphism

$$
\prod_{i=1}^{s} \mathrm{GL}_{2}(k(C)) /\left(\prod_{i=1}^{s} \mathrm{GL}_{2}\left(\mathcal{O}_{P_{i}}\right) \cdot k(C)^{\times}\right) \rightarrow \prod_{i=1}^{\tilde{s}+t} \mathrm{GL}_{2}(k(D)) /\left(\prod_{i=1}^{\tilde{s}+t} \mathrm{GL}_{2}\left(\mathcal{O}_{Q_{i}}\right) \cdot k(D)^{\times}\right),
$$

where the factor $\mathrm{GL}_{2}(k(C))$ for the point $P_{i}$ is mapped diagonally to the factors for $f^{-1}\left(P_{i}\right)$. This morphism is obviously functorial. It is also obviously equivariant with respect to $\mathrm{GL}_{2}(k[C]) \rightarrow \mathrm{GL}_{2}(k[D])$, both groups embedded diagonally into $\mathrm{GL}_{2}(k(C))^{s}$ and $\mathrm{GL}_{2}(k(D))^{\tilde{s}+t}$, respectively.

The cubical structure is slightly more complicated to take care of: a matrix for an elementary transformation

$$
\left(\begin{array}{cc}
\pi_{i}^{a_{i}} & \alpha_{i} \\
0 & 1
\end{array}\right) \text { is mapped to }\left(\begin{array}{cc}
f^{*}\left(\pi_{i}\right)^{a_{i}} & f^{*}\left(\alpha_{i}\right) \\
0 & 1
\end{array}\right)^{g},
$$

and so maps a 1-cube to the diagonal of a $g$-cube, where $g$ is cardinality of $f^{-1}\left(P_{i}\right)$. After suitably subdividing, the above map on vertices extends to a morphism of cell complexes. This proves all the claims for the morphism $f^{*}: \mathfrak{X}_{C} \rightarrow \mathfrak{X}_{D}$. For the parabolic subcomplexes, it then suffices to note that if a cell $\sigma \in \mathfrak{X}_{C}$ has a non-unipotent non-central subgroup in the stabilizer, then the same is true for its image: any non-unipotent non-central element in the stabilizer in $\mathrm{GL}_{2}(k[C])$ will have non-unipotent non-central image in $\mathrm{GL}_{2}(k[D])$. Therefore $f^{*}$ restricts to the parabolic subcomplexes, and all the auxiliary properties are inherited.

\section{LOCAL STRUCTURE OF PARABOLIC SUBCOMPLEXES}

In this section, we work out the local structure of the parabolic subcomplex $\mathfrak{P}_{C}$ resp. its quotient $\Gamma \backslash \mathfrak{P}_{C}$, for $\Gamma$ any of the linear groups $(\mathrm{P}) \mathrm{GL}_{2}(k[C])$ or (P) $\mathrm{SL}_{2}(k[C])$. The general procedure is as follows: for a geometrically split bundle, we completely describe the action of its automorphism group on the link of the corresponding point in the building. The link $\operatorname{Lk}_{\mathfrak{P}}(x)$ of the point $x$ in $\mathfrak{P}_{C}$ is given by those simplices in $\operatorname{Lk}_{\mathfrak{X}}(x)$ which are fixed by a non-unipotent non-central element of the stabilizer of $x$. The link of the image of $x$ in the quotient $\Gamma \backslash \mathfrak{P}_{C}$ is then given by the quotient $\operatorname{Stab}(x ; \Gamma) \backslash \mathrm{Lk}_{\mathfrak{P}}(x)$. These local computations will be used in the next section to describe the global structure of the quotient $\Gamma \backslash \mathfrak{P}_{C}$.

4.1. Action of the automorphism group on the links. We first investigate the action of automorphism groups of rank two bundles on the links of their corresponding points in $\mathfrak{X}_{C}$. Let $\mathcal{E}$ be a vector bundle corresponding to the vertex $x$ in $\mathfrak{X}_{C}$. By Proposition 2.5, the stabilizer of $x$ is essentially determined by the automorphism 
group of the vector bundle $\mathcal{E}$. Combining this with Proposition 3.1, we see that $x$ is in $\mathfrak{P}_{C}$ if and only if $\mathcal{E}$ is geometrically split. Moreover, Proposition 3.1 provides a detailed description of the isomorphism types of possible stabilizer groups.

To obtain a description of the action of those stabilizer groups on the links, recall from Section 2.1 that for $x$ a vertex of the building, the corresponding link is a disjoint union $\bigsqcup_{i=1}^{s} \mathbb{P}^{1}\left(k\left(P_{i}\right)\right)$ with the "complete $s$-partite simplicial structure". The point $x$ is given by specifying $\mathcal{O}_{P_{i}}$-lattices in $V=K^{2}$, or equivalently (using the standard basis of $V$ ) by specifying matrices $M_{i} \in \mathrm{GL}_{2}\left(K_{v_{i}}\right)$, for $i \in\{1, \ldots, s\}$. As in Section 2.1, the link of $x$ can be described in terms of these data: the vertices in the $i$-th component $\mathbb{P}^{1}\left(k\left(P_{i}\right)\right)$ are points corresponding to the tuples

$$
\begin{gathered}
\left(M_{1}, \ldots, M_{i} \cdot\left(\begin{array}{cc}
\pi_{i} & \tilde{\alpha}_{i} \\
0 & 1
\end{array}\right), \ldots, M_{s}\right), \tilde{\alpha}_{i} \in \mathcal{O}_{P_{i}}, \text { and } \\
\left(M_{1}, \ldots, M_{i} \cdot\left(\begin{array}{cc}
\pi_{i}^{-1} & 0 \\
0 & 1
\end{array}\right), \ldots, M_{s}\right) .
\end{gathered}
$$

Note that the point in the building only depends on the $\prod \mathrm{GL}_{2}\left(\mathcal{O}_{P_{i}}\right) \cdot K_{v_{i}}^{\times}$-right coset of the above, in particular, the first line above only depends on the residue class $\alpha_{i}$ of $\tilde{\alpha}_{i}$ in $\mathbb{A}^{1}\left(k\left(P_{i}\right)\right)$. The stabilizer of the point $\left(M_{1}, \cdots, M_{s}\right)$ in the group $\Gamma=(\mathrm{P}) \mathrm{GL}_{2}(k[C])$ or $\Gamma=(\mathrm{P}) \mathrm{SL}_{2}(k[C])$ is then exactly the subgroup of $\Gamma$ leaving invariant the $\prod \mathrm{GL}_{2}\left(\mathcal{O}_{P_{i}}\right) \cdot K_{v_{i}}^{\times}$-right coset of $\left(M_{1}, \ldots, M_{s}\right)$. The next proposition describes the action of the stabilizer on the link by computing explicitly the action of the stabilizer on the right cosets of matrices as given above.

Lemma 4.1. Let $k$ be a field, let $\bar{C}$ be a smooth projective curve over $k$ and set $C=\bar{C} \backslash\left\{P_{1}, \ldots, P_{s}\right\}$. Let $\mathcal{E} \cong \mathcal{L} \oplus \mathcal{L}^{\prime}$ be a split vector bundle over $\bar{C}$, and denote by $x$ the point of $\mathfrak{X}_{C}$ corresponding to $\mathcal{E}$. Denote by $\Gamma(C)$ one of the linear groups $(\mathrm{P}) \mathrm{GL}_{2}(k[C])$ or $(\mathrm{P}) \mathrm{SL}_{2}(k[C])$.

(1) There exist closed points $Q_{1}, \ldots, Q_{t}$ such that the restrictions of both $\mathcal{L}$ and $\mathcal{L}^{\prime}$ to $C^{\prime}=\bar{C} \backslash\left\{P_{1}, \ldots, P_{s}, Q_{1}, \ldots, Q_{t}\right\}$ are trivial.

(2) There is an inclusion of buildings $\mathfrak{X}_{C} \hookrightarrow \mathfrak{X}_{C^{\prime}}$ which is equivariant for the natural homomorphism $\Gamma(C) \rightarrow \Gamma\left(C^{\prime}\right)$ and induces an isomorphism on stabilizers $\operatorname{Stab}(x ; \mathrm{P} \Gamma(C)) \cong \operatorname{Stab}\left(x ; \mathrm{P} \Gamma\left(C^{\prime}\right)\right)$.

(3) As a consequence of (2), the inclusion $\operatorname{Lk}_{\mathfrak{X}_{C}}(x) \hookrightarrow \mathrm{Lk}_{\mathfrak{X}_{C^{\prime}}}(x)$ is equivariant for the action of $\operatorname{Stab}(x ; \Gamma(C))$.

Proof. (1) First note that $\left.\left.\mathcal{L}^{-1}\right|_{C} \cong \mathcal{L}^{\prime}\right|_{C}$ because $\left.\mathcal{E}\right|_{C}$ is trivial. Because $\bar{C}$ is a smooth curve, the usual identification of divisor class group and Picard group allows to write $\mathcal{L} \cong \mathcal{O}_{C}\left(\sum_{i=1}^{s} n_{i} P_{i}+\sum_{j=1}^{t} m_{j} Q_{j}\right)$. The restriction of $\mathcal{L}$ to $C^{\prime}=$ $\bar{C} \backslash\left\{P_{1}, \ldots, P_{s}, Q_{1}, \ldots, Q_{t}\right\}$ is then obviously trivial, cf. also Lemma 2.6.

For $(2)$, we can consider the quotient description of the vertices of the building. The induced map is induced by inclusion of the first $s$ factors:

$$
\begin{gathered}
\prod_{i=1}^{s} \mathrm{GL}_{2}\left(K_{v_{i}}\right) /\left(\mathrm{GL}_{2}\left(\mathcal{O}_{P_{i}}\right) \cdot K_{v_{i}}^{\times}\right) \rightarrow \\
\rightarrow \prod_{i=1}^{s} \mathrm{GL}_{2}\left(K_{v_{i}}\right) /\left(\mathrm{GL}_{2}\left(\mathcal{O}_{P_{i}}\right) \cdot K_{v_{i}}^{\times}\right) \times \prod_{j=1}^{t} \mathrm{GL}_{2}\left(K_{v_{j}}\right) /\left(\mathrm{GL}_{2}\left(\mathcal{O}_{Q_{j}}\right) \cdot K_{v_{j}}^{\times}\right)
\end{gathered}
$$

The map is evidently injective on vertices. Since the building is a cubical complex, i.e., every cube is uniquely determined by its vertices, the map is an inclusion of complexes. As the groups $\Gamma(C)$ and $\Gamma\left(C^{\prime}\right)$ act via diagonal inclusion into the product $\prod \mathrm{GL}_{2}\left(K_{v_{i}}\right)$, the equivariance of the inclusion of buildings is also clear. Similarly, it is clear that the map restricts to a homomorphism of stabilizers. The stabilizers have been described in Proposition 2.5, and from that description it is 
clear that the map induced on stabilizers has to be an isomorphism - the relevant stabilizer groups are directly related to automorphism groups of vector bundles over the curve $\bar{C}$ and independent of the choice of affine subcurve.

(3) is a direct consequence of (2).

Remark 4.2. Note that the isomorphism on stabilizer groups in (2) above only holds for the projective (special) linear groups. In the case of $\mathrm{GL}_{2}$, the stabilizer group can become bigger, cf. Proposition 2.5. However, the $k[C]^{\times}$-part of the stabilizer acts trivially on the link anyway, so this does not affect application of the above lemma to $\mathrm{GL}_{2}(k[C])$.

Proposition 4.3. Let $k$ be a field, let $\bar{C}$ be a smooth projective curve over $k$ and set $C=\bar{C} \backslash\left\{P_{1}, \ldots, P_{s}\right\}$. Let $\mathcal{E} \cong \mathcal{L} \oplus \mathcal{L}^{\prime}$ be a split vector bundle over $\bar{C}$. Denote by $x$ the point of $\mathfrak{X}_{C}$ corresponding to $\mathcal{E}$.

There exist closed points $Q_{1}, \ldots, Q_{t}$ of $C$ such that the image of $x$ in the double quotient

$$
\mathrm{GL}_{2}\left(k\left[C^{\prime}\right]\right) \backslash \prod_{i=1}^{s} \mathrm{GL}_{2}\left(K_{v_{i}}\right) /\left(\mathrm{GL}_{2}\left(\mathcal{O}_{P_{i}}\right) \cdot K_{v_{i}}^{\times}\right) \times \prod_{j=1}^{t} \mathrm{GL}_{2}\left(K_{v_{j}}\right) /\left(\mathrm{GL}_{2}\left(\mathcal{O}_{Q_{j}}\right) \cdot K_{v_{j}}^{\times}\right)
$$

has a representative of the following form:

$$
\left(\left(\begin{array}{cc}
\pi_{1}^{a_{1}} & 0 \\
0 & 1
\end{array}\right), \ldots,\left(\begin{array}{cc}
\pi_{t}^{a_{t}} & 0 \\
0 & 1
\end{array}\right)\right), \quad \sum a_{i} \geq 0 .
$$

(a) Assume $\mathcal{L} \cong \mathcal{L}^{\prime}$, i.e., the stabilizer is conjugate to a diagonally embedded $\mathrm{GL}_{2}(k)$. The action of $\mathrm{GL}_{2}(k)$ on the $i$-th component of the link is the standard action of $\mathrm{GL}_{2}$ on $\mathbb{P}^{1}\left(k\left(P_{i}\right)\right)$, hence the action of $\mathrm{GL}_{2}(k)$ on the link is the diagonal standard action.

(b) Assume $\mathcal{L} ¥ \mathcal{L}^{\prime}$, i.e., the stabilizer is conjugate to the group

$$
\mathrm{B}_{2}\left(a_{1}, \ldots, a_{t}\right)=\left\{\left(\begin{array}{cc}
a & 0 \\
f & b
\end{array}\right) \mid a, b \in k, a b \neq 0, v_{i}(f) \geq-a_{i}\right\} .
$$

There are two cases for the action of the stabilizer on the $i$-th component of the link of $x$ : if $v_{i}(f)=-a_{i}$, then the action is the "standard" action. If $v_{i}(f)>-a_{i}$, the action is trivial.

Proof. We first prove the statement about the double coset representative. Denoting $C^{\prime}=\bar{C} \backslash\left\{P_{1}, \ldots, P_{s}, Q_{1}, \ldots, Q_{t}\right\}$ as in Lemma 4.1, the double quotient written classifies exactly equivalence (rel $\partial C^{\prime}$ ) classes of rank two vector bundles on $\bar{C}$ whose restriction to $C^{\prime}$ is trivial. Therefore, the elements corresponding to $\mathcal{E} \cong \mathcal{L} \oplus \mathcal{L}^{\prime}$ and $\mathcal{L} \otimes\left(\mathcal{L}^{\prime}\right)^{-1} \oplus \mathcal{O}$ are equal in the double quotient. It is easy to see that the double coset representative given corresponds to the vector bundle

$$
\mathcal{O}\left(-\sum_{i=1}^{s} a_{i} P_{i}-\sum_{j=1}^{t} a_{s+j} Q_{j}\right) \oplus \mathcal{O}
$$

Therefore, the statement follows if the $a_{i}$ are chosen such that

$$
\mathcal{O}\left(-\sum_{i=1}^{s} a_{i} P_{i}-\sum_{j=1}^{t} a_{s+j} Q_{j}\right) \cong \mathcal{L} \otimes\left(\mathcal{L}^{\prime}\right)^{-1}
$$

Note that it is necessary to enlarge the set $\left\{P_{1}, \ldots, P_{s}\right\}$ (using Lemma 4.1) because $\mathcal{L} \otimes\left(\mathcal{L}^{\prime}\right)^{-1}$ might not be trivial over $C$, whence it would not correspond to an element in the double quotient $k[C]^{\times} \backslash \prod K_{v_{i}}^{\times} / \prod \mathcal{O}_{P_{i}}^{\times}$.

Next, we explain how to reduce the statements (a) and (b) to statements about the given double coset representative. By Lemma 4.1 (3), the inclusion of links 
$\operatorname{Lk}_{\mathfrak{X}_{C}}(x) \rightarrow \operatorname{Lk}_{\mathfrak{X}_{C^{\prime}}}(x)$ is equivariant for the action of the stabilizer of $x$. To determine this action, it thus suffices to determine the action of $\operatorname{Stab}\left(x ; \mathrm{GL}_{2}(k[C])\right)$ on the vertices of $\mathfrak{X}_{C^{\prime}}$. Moreover, if two points $x$ and $x^{\prime}$ of $\mathfrak{X}_{C^{\prime}}$ are $\mathrm{GL}_{2}\left(k\left[C^{\prime}\right]\right)$ conjugate, then so are their stabilizers as well as the actions of the stabilizers on the respective links. We can therefore replace $x$ by any other representative of the vertices of $\mathrm{GL}_{2}\left(k\left[C^{\prime}\right]\right) \backslash \mathfrak{X}_{C^{\prime}}$. The vertices of the latter quotient are exactly the double cosets in the statement. Summing up, the link $\operatorname{Lk}_{\mathfrak{X}_{C}}(x)$ together with the action of the stabilizer of $x$ can be equivariantly embedded into the link of the chosen representative above in $\mathfrak{X}_{C^{\prime}}$.

Now we come to the prove of part (a). From the above, we have that the stabilizer of $x$ is $\mathrm{GL}_{2}\left(k\left[C^{\prime}\right]\right)$-conjugate to the stabilizer of the double coset $(1, \ldots, 1)$ where all entries are identity matrices. The stabilizer of this double coset inside $\mathrm{GL}_{2}\left(k\left[C^{\prime}\right]\right)$ is obviously $\mathrm{GL}_{2}(k)$, embedded diagonally as constants. Up to right multiplication with elements from $\mathrm{GL}_{2}\left(\mathcal{O}_{P_{i}}\right) \cdot K_{v_{i}}^{\times}$, we find

$$
\begin{gathered}
\left(\begin{array}{ll}
a & b \\
c & d
\end{array}\right) \cdot\left(\begin{array}{cc}
\pi_{i}^{-1} & 0 \\
0 & 1
\end{array}\right)=\left(\begin{array}{cc}
a \pi_{i}^{-1} & b \\
c \pi_{i}^{-1} & d
\end{array}\right) \sim\left(\begin{array}{cc}
\pi_{i} & \frac{a}{c} \\
0 & 1
\end{array}\right), c \neq 0 \\
\left(\begin{array}{cc}
a & b \\
c & d
\end{array}\right) \cdot\left(\begin{array}{cc}
\pi_{i} & \tilde{\alpha} \\
0 & 1
\end{array}\right)=\left(\begin{array}{cc}
a \pi_{i} & a \tilde{\alpha}+b \\
c \pi_{i} & c \tilde{\alpha}+d
\end{array}\right) \sim\left(\begin{array}{cc}
\pi_{i} & \frac{a \tilde{\alpha}+b}{c \tilde{\alpha}+d} \\
0 & 1
\end{array}\right), c \tilde{\alpha}+d \neq 0
\end{gathered}
$$

and the missing cases are dealt with similarly. But this is indeed the standard action of $\mathrm{GL}_{2}(k)$ on $\mathbb{P}^{1}\left(k\left(P_{i}\right)\right)$.

(b) By what was said above, it suffices to compute the stabilizer and its action on the link in the case of the diagonalized double coset representative.

Up to right multiplication by elements of $\mathrm{GL}_{2}\left(\mathcal{O}_{P_{i}}\right) \cdot K_{v_{i}}^{\times}$, we have the following

$$
\left(\begin{array}{ll}
a & 0 \\
f & b
\end{array}\right) \cdot\left(\begin{array}{cc}
\pi_{i}^{a_{i}} & 0 \\
0 & 1
\end{array}\right)=\left(\begin{array}{cc}
a \pi_{i}^{a_{i}} & 0 \\
f \pi_{i}^{a_{i}} & b
\end{array}\right) \sim\left(\begin{array}{cc}
\pi_{i}^{a_{i}} & 0 \\
0 & 1
\end{array}\right),
$$

whenever $v_{i}(f) \geq-a_{i}$. From $\sum a_{i} \geq 0$, it follows that the matrices above are the only ones possibly stabilizing the representative. This shows that the stabilizer is indeed as described.

We now study the action of the stabilizer $\mathrm{B}_{2}\left(a_{1}, \ldots, a_{t}\right)$ on the link of $x$. For the point $\infty$, we have

$$
\left(\begin{array}{ll}
a & 0 \\
f & b
\end{array}\right)\left(\begin{array}{cc}
\pi_{i}^{a_{i}-1} & 0 \\
0 & 1
\end{array}\right)=\left(\begin{array}{cc}
a \pi_{i}^{a_{i}-1} & 0 \\
f \pi_{i}^{a_{i}-1} & b
\end{array}\right)
$$

and there are two cases to consider: if $v_{i}(f)=-a_{i}$, then $v_{i}\left(f \pi_{i}^{a_{i}-1}\right)=-1$, and we can rewrite the matrix using right multiplication with $\mathrm{GL}_{2}\left(\mathcal{O}_{P_{i}}\right) \cdot K_{v_{i}}^{\times}$to the form

$$
\left(\begin{array}{cc}
\pi_{i}^{a_{i}+1} & g \\
0 & 1
\end{array}\right)
$$

where $v(g)=a_{i}$ and the leading term is $\frac{a}{f_{0}}$ with $f_{0}$ the leading term of $f$ (as in the constant case). On the other hand, if $v_{i}(f)>-a_{i}$, then $v_{i}\left(f \pi_{i}^{a_{i}-1}\right) \geq 0$, and right multiplication allows to rewrite this to $\operatorname{diag}\left(\pi_{i}^{a_{i}-1}, 1\right)$. In this case, the action is trivial. For any other point $\alpha \in \mathbb{P}^{1}\left(k\left(\mathbb{P}^{1}\right)\right) \backslash\{\infty\}$, we consider

$$
\left(\begin{array}{cc}
1 & 0 \\
f & 1
\end{array}\right)\left(\begin{array}{cc}
\pi_{i}^{a_{i}+1} & \pi_{i}^{a_{i}} \tilde{\alpha} \\
0 & 1
\end{array}\right)=\left(\begin{array}{cc}
\pi_{i}^{a_{i}+1} & \pi_{i}^{a_{i}} \tilde{\alpha} \\
f \pi_{i}^{a_{i}+1} & f \pi_{i}^{a_{i}} \tilde{\alpha}+1
\end{array}\right) .
$$

The restriction $a=b=1$ is only for simplification of exposition. In the case $v_{i}(f)=-a_{i}$, we can use right multiplication to transform the matrix to

$$
\left(\begin{array}{cc}
\pi_{i}^{a_{i}+1} & \pi_{i}^{a_{i}} \cdot \frac{\tilde{\alpha}}{1+f \pi_{i}^{a_{i}} \tilde{\alpha}} \\
0 & 1
\end{array}\right)
$$


We can use further right multiplication to transform $\frac{\tilde{\alpha}}{1+f \pi_{i}^{a_{i}} \tilde{\alpha}}$ to $\frac{\tilde{\alpha}}{1+c \tilde{\alpha}}$ with $c$ the constant term of $f \pi_{i}^{a_{i}}$. If $v_{i}(f)>-a_{i}$, then this constant term is 0 , and the action is trivial.

For $v_{i}(f)=-a_{i}=0$, this is the standard action of the Borel $\mathbb{B}_{2}(k)$ on $\mathbb{P}^{1}\left(k\left(P_{i}\right)\right)$. For $v_{i}(f)=-a_{i} \neq 0$, the action is still the same, only that $f$ acts via its constant coefficient. If $v_{i}(f)>-a_{i}$, then the action is trivial. The claim is proved.

Remark 4.4. A similar result holds for bundles which are indecomposable but geometrically split. Let $\mathcal{E}$ be such a bundle, with corresponding point $x \in \mathfrak{X}_{C}$, and let $L / k$ be a splitting field for the bundle $\mathcal{E}$. Then the morphism of curves $\phi: \bar{C} \times_{k} L \rightarrow \bar{C}$ induces a morphism of buildings $\mathfrak{X}_{C} \hookrightarrow \mathfrak{X}_{C \times{ }_{k} L}$ which embeds $\mathfrak{X}_{C}$ as the $\operatorname{Gal}(L / k)$-invariants into $\mathfrak{X}_{C \times{ }_{k} L}$. The results of Proposition 4.3 above then imply that the action of the stabilizer of $x$ on $\operatorname{Lk}_{\mathfrak{X}_{C}}(x)$ is also the standard one, coming from the embedding of $\operatorname{Stab}\left(x ; \mathrm{GL}_{2}(k[C])\right)$ as $\mathrm{Gal}(L / k)$-invariants of $\operatorname{Stab}\left(x ; \mathrm{GL}_{2}(L[C])\right)$. For this and other reasons, our main results are restricted to the case of algebraically closed base fields.

4.2. Local structure. Having determined the action of vertex stabilizers on links, we are now ready to describe the local structure of the quotients $\Gamma \backslash \mathfrak{P}_{C}$ as subcomplexes of the respective quotients of $\Gamma \backslash \mathfrak{X}_{C}$, where as usual $\Gamma$ is one of the linear groups $(\mathrm{P}) \mathrm{GL}_{2}(k[C])$ or $(\mathrm{P}) \mathrm{SL}_{2}(k[C])$. For a point $x \in \mathfrak{X}_{C}$, the link $\mathrm{Lk}_{\Gamma \backslash \mathfrak{P}_{C}}$ is the quotient $\operatorname{Stab}(x ; \Gamma) \backslash \mathrm{Lk}_{\mathfrak{P}_{C}}(x)$. The following proposition now uses Proposition 4.3 to determine $\operatorname{Lk}_{\mathfrak{P}_{C}}(x)$ as well as its quotient modulo $\operatorname{Stab}(x ; \Gamma)$.

Proposition 4.5. Let $k$ be an algebraically closed field, let $\bar{C}$ be a smooth projective curve over $k$ and denote $C=\bar{C} \backslash\left\{P_{1}, \ldots, P_{s}\right\}$. Let $\mathcal{E} \cong \mathcal{L} \oplus \mathcal{L}^{\prime}$ be a split rank two vector bundle, and let $x$ be the corresponding point in the building $\mathfrak{X}_{C}$. Denote by $\Gamma$ one of the linear groups $(\mathrm{P}) \mathrm{GL}_{2}(k[C])$ or $(\mathrm{P}) \mathrm{SL}_{2}(k[C])$.

Denote by $\mathfrak{A}_{x}$ the intersection of an apartment $\mathfrak{A}$ with $\operatorname{Lk}_{\mathfrak{X}_{C}}(x)$.

(a) If $\mathcal{L}^{\prime} \cong \mathcal{L}^{-1}$, then $\operatorname{Lk}_{\Gamma \backslash \mathfrak{P}}(x)$ is isomorphic to the quotient of $\mathfrak{A}_{x}$ modulo the antipodal $\mathbb{Z} / 2$-action. The star $\operatorname{St}_{\Gamma \backslash \mathfrak{P}}(x)$ is isomorphic to a cone over (a cubical complex of the homotopy type of) $\mathbb{R P}^{s-1}$. The stabilizer of $x$ is $\Gamma(k)$, everything else is stabilized (up to a unipotent group) by a maximal torus of $\Gamma$.

(b) If $\mathcal{L}^{\prime} ¥ \mathcal{L}^{-1}$, then $\operatorname{Lk}_{\Gamma \backslash \mathfrak{P}}(x)$ is isomorphic to $\mathfrak{A}_{x}$. The stabilizer is (up to a unipotent group) a maximal torus in $\Gamma(k)$.

Proof. Recall that for a field $F$, the set $\mathbb{P}^{1}(F)$ is a building of type $A_{1}$. The group $\mathrm{GL}_{2}(F)$ acts in the standard way on $\mathbb{P}^{1}(F)$. The standard apartment is the subset $\{0, \infty\} \subseteq \mathbb{P}^{1}(F)$, its setwise stabilizer in $\mathrm{GL}_{2}(F)$ is the normalizer $\mathrm{N}_{2}(F)$ of the standard maximal torus $\mathrm{T}_{2}(F)$, its pointwise stabilizer is the standard maximal torus $\mathrm{T}_{2}(F)$.

Now we consider the "complete $n$-partite simplicial complex" $\operatorname{Lk}_{\mathfrak{X}_{C}}(x)$ on the disjoint union $\bigsqcup_{i=1}^{s} \mathbb{P}^{1}(k)$, with the diagonal action of $\mathrm{GL}_{2}(k)$. Note that $\mathfrak{A}_{x}$ is conjugate to the full subcomplex of $\operatorname{Lk}_{\mathfrak{X}_{C}}(x)$ spanned by $\bigsqcup_{i=1}^{s}\left\{0_{i}, \infty_{i}\right\} \subseteq \bigsqcup_{i=1}^{s} \mathbb{P}^{1}(k)$. It is easy to see that the setwise stabilizer of $\mathfrak{A}_{x}$ is conjugate to the normalizer $\mathrm{N}_{2}(k)$ and the pointwise stabilizer is conjugate to the standard maximal torus $\mathrm{T}_{2}(k)$. Note that $\mathfrak{A}_{x}$ is a triangulation of the $(s-1)$-sphere. Moreover, the Weyl group $\mathrm{N}_{2}(k) / \mathrm{T}_{2}(k) \cong \mathbb{Z} / 2$ acts on $\mathfrak{A}_{x}$ by the antipodal action.

(a) By Proposition 4.3, we have $\operatorname{Stab}(x ; \Gamma)=\Gamma(k)$ acting via the diagonal standard action on the link $\bigsqcup_{i=1}^{s} \mathbb{P}^{1}(k)$. A simplex $\sigma \in \operatorname{Lk}_{\mathfrak{X}_{C}}(x)$ is contained in $\operatorname{Lk}_{\mathfrak{P}_{C}}(x)$ if its stabilizer in $\mathrm{GL}_{2}(k)$ contains a non-central non-unipotent element. The latter is equivalent to $\sigma$ being stabilized by some maximal torus of $\mathrm{GL}_{2}(k)$. But this is the case precisely if the corresponding simplex is $\mathrm{GL}_{2}(k)$-conjugate to one in $\mathfrak{A}_{x}$. We see that every simplex in $\operatorname{Lk}_{\mathfrak{P}_{C}}(x)$ is $\mathrm{GL}_{2}(k)$-conjugate to one in $\mathfrak{A}_{x}$. As remarked 
above, the setwise stabilizer of the standard apartment is the normalizer of the maximal torus corresponding to $\mathfrak{A}_{x}$, the pointwise stabilizer of the standard apartment is the maximal torus itself, and the Weyl group acts via the antipodal action. This proves the claim about the link. The claim about the star follows, because the star is the cone over the link. The maximal torus stabilizes $\mathfrak{A}_{x}$ pointwise, so each simplex in $\operatorname{Lk}_{\Gamma \backslash \mathfrak{P}_{C}}(x)$ is stabilized at least by the maximal torus. As there are no simplices stabilized by $\mathrm{GL}_{2}(k)$, the claim follows from the knowledge of stabilizers in Proposition 3.1.

(b) By Proposition 4.3, we have $\operatorname{Stab}\left(x ; \mathrm{GL}_{2}(k[C])\right) \cong k^{n} \rtimes \mathrm{T}_{2}(k)$. The maximal torus acts via the diagonal standard action on the link $\bigsqcup_{i=1}^{s} \mathbb{P}^{1}(k)$. Fixing a section of $k^{n} \rtimes \mathrm{T}_{2}(k) \rightarrow \mathrm{T}_{2}(k)$, there are exactly two fixed points. Any two such choices of sections yield actions which are conjugate (by the unipotent radical) to the action with fixed points exactly 0 and $\infty$ in each disjoint summand $\mathbb{P}^{1}(k)$. Then also the simplices connecting these points are stabilized by the maximal torus. Furthermore, any simplex which is stabilized by some non-unipotent element is stabilized by some such maximal torus. Summing up, we see that every simplex in $\operatorname{Lk}_{\mathfrak{P}_{C}}(x)$ is $\operatorname{Stab}\left(x ; \mathrm{GL}_{2}(k[C])\right)$-conjugate to a simplex in $\mathfrak{A}_{x}$, and $\mathfrak{A}_{x}$ is a strict fundamental domain for the $\operatorname{Stab}\left(x ; \mathrm{GL}_{2}(k[C])\right)$-action on $\mathrm{Lk}_{\mathfrak{P}_{C}}(x)$. This proves the claim.

The same statements also are true for $\mathrm{SL}_{2}(k[C])$ instead of $\mathrm{GL}_{2}(k[C])$ used above.

Remark 4.6. The link $\operatorname{Lk}_{\mathfrak{P}_{C}}(x)$ is the preimage of these links under the projection $\mathfrak{P}_{C} \rightarrow \Gamma \backslash \mathfrak{P}_{C}$. However, it is more difficult to describe explicitly due to unipotent subgroups in the stabilizer of $x$. We ignored these in the local description of $\Gamma \backslash \mathfrak{P}_{C}$ because they will not be visible in the homology computations anyway.

Remark 4.7. The reason why we restricted to algebraically closed base fields in Proposition 4.5 is the following: although we have identified in Proposition 4.3 that the action of $\mathrm{GL}_{2}(k)$ on $\mathbb{P}^{1}\left(k\left(P_{i}\right)\right)$ is the standard one, this does not allow to give an easy description of the quotient $\operatorname{Stab}(x ; \Gamma) \backslash \mathrm{Lk}_{\mathfrak{P}}(x)$.

The phenomenon is already visible in the case where $s=1$ and $\left[k\left(P_{1}\right): k\right]=2$. The action of $\mathrm{GL}_{2}(k)$ on $\mathbb{P}^{1}\left(k\left(P_{1}\right)\right)$ is no longer transitive but has three orbits, one orbit isomorphic to $\mathbb{P}^{1}(k)$, any of the two other orbits is a symmetric space generalizing the upper half plane $\mathbb{H}$. The stabilizer of points in $\mathbb{P}^{1}(k)$ is a Borel subgroup, the stabilizer of points in the other orbits is a rank one non-split torus.

In general, depending on the size of $k\left(P_{i}\right)$ and the number of degree 2 subfields, there can be many relevant non-split tori in $\mathrm{GL}_{2}(k)$ fixing points in $\mathbb{P}^{1}\left(k\left(P_{i}\right)\right)$. The description of the quotient $\operatorname{Stab}(x ; \Gamma) \backslash \mathrm{Lk}_{\mathfrak{P}}(x)$ then strongly depends on the extension $k\left(P_{i}\right) / k$. In any case, the quotient tends to be much more complicated than in the algebraically closed case handled in Proposition 4.5.

\section{Global structure of parabolic subcomplexes}

After having determined the local structure of the parabolic subcomplex $\Gamma \backslash \mathfrak{P}_{C}$, we will now turn to the investigation of the global structure. The main results state that $\Gamma \backslash \mathfrak{P}_{C}$ is a disjoint union of subcomplexes, indexed by classes of line bundles over $C$, and the structure of the subcomplex associated to the line bundle $\mathcal{L}$ can be explicitly described: if $\mathcal{L} \approx \mathcal{L}^{-1}$ then it is a torus related to the classifying space of a maximal torus in $\mathrm{GL}_{2}(K)$, and if $\mathcal{L} \cong \mathcal{L}^{-1}$ it is a slightly more complicated space related to the classifying space of the normalizer of a maximal torus in $\mathrm{GL}_{2}(K)$.

The topology of the quotient $\mathrm{GL}_{2}(k[C]) \backslash \mathfrak{X}_{C}$ - and in particular the homotopy type at infinity - was studied already by Stuhler in Stu76] and Stu80, in the case where $k=\mathbb{F}_{q}$. The results below can be seen as a version of his work: our results work over algebraically closed base fields $k$ and describe a part of $\mathrm{GL}_{2}(k[C]) \backslash \mathfrak{X}_{C}$ 
slightly larger than a neighbourhood of infinity. The method of proof here is still the one already employed by Stuhler: we will write down model complexes, and the local computations from Section 4 will imply that the model complexes are isomorphic to the components of $\Gamma \backslash \mathfrak{P}_{C}$.

\subsection{Line bundles and connected components.}

Definition 5.1. Let $k$ be a field, let $\bar{C}$ be a smooth projective curve and denote $C=\bar{C} \backslash\left\{P_{1}, \ldots, P_{s}\right\}$. We define $\mathcal{K}(C)$ to be the quotient of the Picard group $\operatorname{Pic}(C)$ modulo the involution $\iota: \mathcal{L} \mapsto \mathcal{L}^{-1}$.

As the notation suggests, the set $\mathcal{K}(C)$ is both related to the Kummer variety associated to the curve $\bar{C}$ as well as the set of komponents of $\Gamma \backslash \mathfrak{P}_{C}$ :

Lemma 5.2. Let $k$ be an algebraically closed field, let $\bar{C}$ be a smooth projective curve and denote $C=\bar{C} \backslash\left\{P_{1}, \ldots, P_{s}\right\}$. Denote by $\Gamma$ one of the groups $(\mathrm{P}) \mathrm{GL}_{2}(k[C])$ or $(\mathrm{P}) \mathrm{SL}_{2}(k[C])$. Then we have a bijection

$$
\pi_{0}\left(\Gamma \backslash \mathfrak{P}_{C}\right) \cong \mathcal{K}(C) .
$$

Proof. The set $\pi_{0}$ is defined by taking the set of vertices of $\Gamma \backslash \mathfrak{P}_{C}$ and dividing by the equivalence relation generated by 1-cells of $\Gamma \backslash \mathfrak{P}_{C}$.

We first write down a map $\left(\Gamma \backslash \mathfrak{P}_{C}\right)_{0} \rightarrow \mathcal{K}(C)$ : by Definition 3.6, Proposition 3.1 and our assumption that $k$ is algebraically closed, any vertex $x$ of $\Gamma \backslash \mathfrak{P}_{C}$ corresponds to a split vector bundle $\mathcal{E} \cong \mathcal{L} \oplus \mathcal{L}^{\prime}$. Recall from Proposition 2.3 that

$$
\operatorname{det} \mathcal{E} \cong \mathcal{L} \otimes \mathcal{L}^{\prime} \cong \mathcal{O}\left(a_{1} P_{1}\right) \otimes \cdots \otimes \mathcal{O}\left(a_{s} P_{s}\right)
$$

for suitable $a_{i} \in \mathbb{Z}$. Using Lemma 2.6, we see that the assignment

$$
\left(\Gamma \backslash \mathfrak{P}_{C}\right)_{0} \rightarrow \mathcal{K}(C): x=\left[\mathcal{L} \oplus \mathcal{L}^{\prime}\right] \mapsto[\mathcal{L}]
$$

is well-defined. It does not depend on the choice of ( rel $\partial C$ )-equivalence class of a representative $\mathcal{E}$ of $x$ and does not depend on the splitting.

The map is clearly surjective: for any element $l \in \mathcal{K}(C)$, we can find a line bundle $\mathcal{L}$ on $\bar{C}$ such that $l=\left\{\left.\mathcal{L}\right|_{C},\left.\mathcal{L}^{-1}\right|_{C}\right\}$. Then $\mathcal{L} \oplus \mathcal{L}^{-1}$ is a vector bundle on $\bar{C}$ with trivial determinant, hence corresponds to a point $x \in \Gamma \backslash \mathfrak{P}_{C}$ which is mapped to $l$ by the map described above.

It suffices to show injectivity. Let $x_{1}$ and $x_{2}$ be two points in $\Gamma \backslash \mathfrak{P}_{C}$ corresponding to vector bundles $\mathcal{E}_{1}$ and $\mathcal{E}_{2}$ which are both mapped to $l \in \mathcal{K}(C)$. This means that we have splittings

$$
\mathcal{E}_{1} \cong \mathcal{L}_{1} \oplus \mathcal{L}_{1}^{\prime}, \quad \mathcal{E}_{2} \cong \mathcal{L}_{2} \oplus \mathcal{L}_{2}^{\prime},
$$

with $\left.\mathcal{L}_{1}\right|_{C} \cong\left(\left.\mathcal{L}_{2}\right|_{C}\right)^{ \pm 1}$. Up to equivalence (rel $\partial C$ ) and switching summands, we can assume $\mathcal{L}_{1} \cong \mathcal{L}_{2}$. But then we have

$$
\mathcal{L}_{1}^{\prime} \otimes\left(\mathcal{L}_{2}^{\prime}\right)^{-1} \cong \mathcal{O}\left(a_{1} P_{1}\right) \otimes \cdots \otimes \mathcal{O}\left(a_{s} P_{s}\right)
$$

We need to find a chain of 1 -cubes in $\Gamma \backslash \mathfrak{P}_{C}$ connecting $\mathcal{E}_{1}$ to $\mathcal{E}_{2}$. It is easy to find a chain of elementary transformations connecting $\mathcal{O}\left(a_{1} P_{1}\right) \otimes \cdots \otimes \mathcal{O}\left(a_{s} P_{s}\right)$ to the trivial line bundle, noting that $\mathcal{O}(-P) \hookrightarrow \mathcal{O}$ is an inclusion of line bundles whose quotient is a torsion sheaf of length one on the curve $\bar{C}$. This provides a chain of 1-cubes in $\Gamma \backslash \mathfrak{X}_{C}$ connecting $\mathcal{E}_{1}$ and $\mathcal{E}_{2}$.

We claim that this chain is contained in $\mathfrak{P}_{C}$. Let $\mathcal{L}$ be any line bundle and consider the two bundles $\mathcal{L} \oplus \mathcal{L}^{-1} \otimes \mathcal{O}(P)$ and $\mathcal{L} \oplus \mathcal{L}^{-1}$. We can choose a double coset representative of $\mathcal{L} \oplus \mathcal{L}^{-1}$ which only contains diagonal matrices. In particular, the stabilizer contains the diagonal maximal torus. The elementary transformation from $\mathcal{L} \oplus \mathcal{L}^{-1}$ to $\mathcal{L} \oplus \mathcal{L}^{-1} \otimes \mathcal{O}(P)$ is then given by the diagonal matrix $\operatorname{diag}(1, \pi)$ where $\pi$ is a uniformizer at $P$. In particular, this elementary transformation is compatible with the action of the diagonal torus. In other words, identifying the 
link of $\mathcal{L} \oplus \mathcal{L}^{-1}$ with $\mathbb{P}^{1}$ with the standard action of the diagonal torus, the above elementary transformation corresponds to the point $\infty$ which is fixed by the diagonal torus. Therefore, the 1-cube corresponding to the above elementary transformation actually lies in the subcomplex $\Gamma \backslash \mathfrak{P}_{C}$. The general assertion is proved in just the same way, only more notational effort is required to keep track of all the coefficients $a_{i}$. Summing up, the path constructed between $\mathcal{E}_{1}$ and $\mathcal{E}_{2}$ lies inside $\Gamma \backslash \mathfrak{P}_{C}$, and we have thus shown that the map $\pi_{0}\left(\Gamma \backslash \mathfrak{P}_{C}\right) \rightarrow \mathcal{K}(C)$ is injective.

Remark 5.3. For a number field $K$, one considers the action of $\mathrm{SL}_{2}\left(\mathcal{O}_{K}\right)$ on its corresponding symmetric space $\mathfrak{X}$. In this situation, there is a well-known bijection between the cusps of the locally symmetric space $\mathrm{SL}_{2}\left(\mathcal{O}_{K}\right) \backslash \mathfrak{X}$ and the class group of $K$, going back (at least) to Siegel. The above computation of the connected components of $\Gamma \backslash \mathfrak{X}_{C}$ is a variation on this. The obvious change is that the class group has been replaced by the Picard group of the curve $C$. A non-obvious change is the appearance of the involution $\iota$. This comes from the fact that for each line bundle $\mathcal{L}$ with $\mathcal{L} \neq \mathcal{L}^{-1}$, there are two components of the homotopy type at infinity, belonging to $\mathcal{L}$ and $\mathcal{L}^{-1}$, which belong to the same connected component of $\mathfrak{P}_{C}$.

5.2. Model complexes of groups. Now we come to the description of the connected components of $\Gamma \backslash \mathfrak{P}_{C}$. There are two cases, depending on the line bundle $\mathcal{L}$ indexing the component: if $\mathcal{L} \neq \mathcal{L}^{-1}$ then the component is related to a "maximal torus of $\Gamma$ ", and if $\mathcal{L} \cong \mathcal{L}^{-1}$ then the component is related to the "normalizer of a maximal torus of $\Gamma$ ". The results are proved by first defining models for the corresponding quotients and then provide isomorphisms to the respective connected components.

Definition 5.4. Let $\mathbb{Z}^{s} \subseteq \mathbb{R}^{s}$ be the standard lattice. Denote by $\mathcal{Z}^{s}$ the cubical complex generated by it. $\bar{W}$ e define the groups

$$
\mathcal{T}=\left\{\left(a_{1}, \ldots, a_{s}\right) \in \mathbb{Z}^{s} \mid \sum_{i=1}^{s} a_{i}\left[P_{i}\right]=0 \text { in } \operatorname{Pic}(\bar{C})\right\}, \quad \text { and } \quad \mathcal{S} \mathcal{T}=\mathcal{T}^{2} .
$$

The groups $\mathcal{T}$ and $\mathcal{S} \mathcal{T}$ act on $\mathbb{R}^{s}$ by translations and preserve the standard lattice $\mathbb{Z}^{s}$. Therefore, the translation actions of $\mathcal{T}$ and $\mathcal{S} \mathcal{T}$ induces respective actions on the cubical complex $\mathcal{Z}^{s}$.

$W$ define the groups $\mathcal{N}$ (resp. $\mathcal{S N}$ ) to be the subgroups of the euclidean group $\mathrm{E}(s)$ generated by $\mathcal{T}$ (resp. $\mathcal{S} \mathcal{T}$ ) and all point inversions in the points $\left(a_{1}, \ldots, a_{s}\right)$ for which $\sum_{i=1}^{s} a_{i}\left[P_{i}\right]=0$ in $\operatorname{Pic}(\bar{C})$.

Remark 5.5. Note that the complex $\mathcal{Z}^{s} / \mathcal{T}$ already appears in Stu80] and is used there for the description of the homotopy type of $\mathrm{PGL}_{2}(k[C]) \backslash \mathfrak{X}_{C}$ at infinity.

We note that the group $\mathcal{T}$ is a free abelian group whose rank equals the rank of the kernel of $\phi$ in Lemma 2.6, and in particular is isomorphic to the group of non-constant units in $k[C]$. There are many reasons for calling this group $\mathcal{T}$ - it is realized as a subgroup of translations, it is a subgroup of a maximal torus of $\mathrm{GL}_{2}(K)$, and the quotient of $\mathcal{Z}^{s} / \mathcal{T}$ has the homotopy type of a torus.

The next results will describe the structure of the connected components of $\Gamma \backslash \mathfrak{P}_{C}$ as quotients of apartments modulo suitable torus- or normalizer-like groups.

Lemma 5.6. Let $k$ be an algebraically closed field, let $\bar{C}$ be a smooth projective curve and denote $C=\bar{C} \backslash\left\{P_{1}, \ldots, P_{s}\right\}$. Let $\mathcal{L}$ be a line bundle on $\bar{C}$. There exists an apartment $\mathfrak{A}_{\mathcal{L}}$ of $\mathfrak{X}_{C}$ and a morphism of cubical complexes $\phi_{\mathcal{L}}: \mathcal{Z}^{s} \rightarrow \mathfrak{A}_{\mathcal{L}}$ such that

(1) $\mathfrak{A}_{\mathcal{L}}$ is contained in $\mathfrak{P}_{C}$, 
(2) the 0 -cells of the image of $\mathfrak{A}_{\mathcal{L}}$ in $\mathrm{GL}_{2}(k[C]) \backslash \mathfrak{X}_{C}$ correspond to equivalence (rel $\partial C$ ) classes of rank two bundles of the form

$$
\left(\mathcal{L} \otimes \mathcal{O}\left(a_{1} P_{1}\right) \otimes \cdots \otimes \mathcal{O}\left(a_{s} P_{s}\right)\right) \oplus \mathcal{L}^{-1}, \quad a_{i} \in \mathbb{Z},
$$

(3) $\phi_{\mathcal{L}}$ is an isomorphism.

Proof. Recall the double quotient description of the 0 -cells of $\mathrm{GL}_{2}(k[C]) \backslash \mathfrak{X}_{C}$ :

$$
\mathrm{GL}_{2}(k[C]) \backslash \prod_{i=1}^{s} \mathrm{GL}_{2}(K) / \prod_{i=1}^{s}\left(\mathrm{GL}_{2}\left(\mathcal{O}_{P_{i}}\right) \cdot K^{\times}\right) .
$$

Let $\left(M_{1}, \ldots, M_{s}\right) \in \mathrm{GL}_{2}(K)^{s}$ be a representative of the double coset corresponding to the bundle $\mathcal{L} \oplus \mathcal{L}^{-1}$. Denoting by $\mathrm{T}_{2}(K)$ the diagonal maximal torus of $\mathrm{GL}_{2}(K)$, we consider the maximal torus

$$
\mathrm{T}(\mathcal{L})=\left(M_{1} \mathrm{~T}_{2}(K) M_{1}^{-1}, \ldots, M_{s} \mathrm{~T}_{2}(K) M_{s}^{-1}\right) \subset \mathrm{GL}_{2}(K)^{s} .
$$

We define $\mathfrak{A}_{\mathcal{L}}$ to be the full cubical subcomplex of $\mathfrak{X}_{C}$ whose 0 -cubes are the $\mathrm{T}(\mathcal{L})$ orbit of $\left(M_{1}, \ldots, M_{s}\right)$. By construction this is an apartment of $\mathfrak{X}_{C}$, corresponding to a choice of frame in each of the $s$ copies of $K^{2}$.

(1) We see that the subgroup $\left(M_{1} \mathrm{~T}_{2}(k) M_{1}^{-1}, \ldots, M_{s} \mathrm{~T}_{2}(k) M_{s}^{-1}\right) \subset \mathrm{T}(\mathcal{L})$ preserves lattice classes in $\mathfrak{A}_{\mathcal{L}}$ because $\mathrm{T}_{2}(k) \subseteq \mathrm{GL}_{2}\left(\mathcal{O}_{P_{i}}\right)$. In particular, this subgroup stabilizes $\mathfrak{A}_{\mathcal{L}}$ pointwise. On the other hand, because $\left(M_{1}, \ldots, M_{s}\right)$ represents a split rank two bundle $\mathcal{L} \oplus \mathcal{L}^{-1}$, the point $\left(M_{1}, \ldots, M_{s}\right)$ is stabilized by a maximal torus of $\mathrm{GL}_{2}(k)$. From Proposition 4.3 we know that the action of the stabilizer of $\mathcal{L} \oplus \mathcal{L}^{-1}$ on the link is such that the stabilizer contains a maximal torus which fixes pointwise the intersection of $\mathfrak{A}_{\mathcal{L}}$ with the link $\operatorname{Lk}_{\mathfrak{X}_{C}}\left(\mathcal{L} \oplus \mathcal{L}^{-1}\right)$. In particular, all the vertices in the link $\operatorname{Lk}_{\mathfrak{A}_{\mathcal{L}}}\left(\mathcal{L} \oplus \mathcal{L}^{-1}\right)$ have the same stabilizer as $\mathcal{L} \oplus \mathcal{L}^{-1}$. An inductive argument shows that this is true for all vertices in $\mathfrak{A}_{\mathcal{L}}$. This implies that the intersection

$$
\left(M_{1} \mathrm{~T}_{2}(k) M_{1}^{-1}, \ldots, M_{s} \mathrm{~T}_{2}(k) M_{s}^{-1}\right) \cap \mathrm{GL}_{2}(k[C])
$$

contains a maximal torus of $\mathrm{GL}_{2}(k)$. Then all cubes of $\mathfrak{A}_{\mathcal{L}}$ are stabilized by this, and we see that $\mathfrak{A}_{\mathcal{L}} \subset \mathfrak{P}_{C}$.

(2) is easy to see: up to $\prod_{i=1}^{s}\left(\mathrm{GL}_{2}\left(\mathcal{O}_{P_{i}}\right) \cdot K^{\times}\right)$, the elements of $\mathrm{T}_{2}^{s}$ are of the form

$$
A=\left(\left(\begin{array}{cc}
\pi_{1}^{a_{1}} & 0 \\
0 & 1
\end{array}\right), \cdots,\left(\begin{array}{cc}
\pi_{s}^{a_{s}} & 0 \\
0 & 1
\end{array}\right)\right)
$$

But then the elements of $\mathrm{T}(\mathcal{L}) \cdot\left(M_{1}, \ldots, M_{s}\right)$ are those of the form

$$
\left(M_{1} \cdot\left(\begin{array}{cc}
\pi_{1}^{a_{1}} & 0 \\
0 & 1
\end{array}\right), \cdots, M_{s} \cdot\left(\begin{array}{cc}
\pi_{s}^{a_{s}} & 0 \\
0 & 1
\end{array}\right)\right)
$$

and these are exactly representatives for bundles of the form

$$
\left(\mathcal{L} \otimes \mathcal{O}\left(-a_{1} P_{1}\right) \otimes \cdots \otimes \mathcal{O}\left(-a_{s} P_{s}\right)\right) \oplus \mathcal{L}^{-1}, \quad a_{i} \in \mathbb{Z} .
$$

Now the morphism $\phi_{\mathcal{L}}: \mathcal{Z}^{s} \rightarrow \mathfrak{A}_{\mathcal{L}}$ is defined on 0 -cubes as

$$
\left(a_{1}, \ldots, a_{s}\right) \mapsto\left(M_{1} \cdot\left(\begin{array}{cc}
\pi_{1}^{a_{1}} & 0 \\
0 & 1
\end{array}\right), \cdots, M_{s} \cdot\left(\begin{array}{cc}
\pi_{s}^{a_{s}} & 0 \\
0 & 1
\end{array}\right)\right),
$$

and we have seen above that this assignment indeed takes vertices to 0 -cubes in $\mathfrak{A}_{\mathcal{L}}$. It is also easy to see that if vertices span a cube in $\mathcal{Z}^{s}$, then their $\phi_{\mathcal{L}}$-images span a cube in $\mathfrak{A}_{\mathcal{L}}$. Therefore, the above definition of $\phi_{\mathcal{L}}$ indeed provides a morphism of cubical complexes. (3) follows because $\mathfrak{A}_{\mathcal{L}}$ is a Coxeter complex for the affine Weyl group $\mathbb{Z}^{s} \rtimes \mathbb{Z} / 2^{s}$, and hence the morphism $\phi_{\mathcal{L}}$ has an obvious inverse mapping

$$
\left(M_{1} \cdot\left(\begin{array}{cc}
\pi_{1}^{a_{1}} & 0 \\
0 & 1
\end{array}\right), \cdots, M_{s} \cdot\left(\begin{array}{cc}
\pi_{s}^{a_{s}} & 0 \\
0 & 1
\end{array}\right)\right) \mapsto\left(a_{1}, \ldots, a_{s}\right) .
$$


Proposition 5.7. Let $k$ be an algebraically closed field, let $\bar{C}$ be a smooth projective curve and denote $C=\bar{C} \backslash\left\{P_{1}, \ldots, P_{s}\right\}$. Let $\mathcal{L}$ be a line bundle on $\bar{C}$ such that $\left.\left.\mathcal{L}\right|_{C} \nRightarrow \mathcal{L}^{-1}\right|_{C}$.

(1) The connected component of $\mathrm{GL}_{2}(k[C]) \backslash \mathfrak{P}_{C}$ containing the vector bundle $\mathcal{L} \oplus \mathcal{L}^{-1}$ is isomorphic to $\mathcal{Z}^{s} / \mathcal{T}$. The stabilizers of cells are of the form $\left(k^{n} \rtimes\right.$ $\left.\left(k^{\times}\right)^{2}\right) \times_{k} \times k[C]^{\times}$for some $n$ depending on the cell, i.e., up to the unipotent radical all the cells have stabilizer $\mathrm{T} \times_{k \times} k[C]^{\times}$where $\mathrm{T}$ is a maximal torus in $\mathrm{GL}_{2}(k)$.

(2) The connected component of $\mathrm{SL}_{2}(k[C]) \backslash \mathfrak{P}_{C}$ containing the vector bundle $\mathcal{L} \oplus \mathcal{L}^{-1}$ is isomorphic to $\mathcal{Z}^{s} / \mathcal{S T}$. The stabilizers of cells are of the form $k^{n} \rtimes k^{\times}$for some $n$ depending on the cell, i.e., up to the unipotent radical all the cells have stabilizer a maximal torus in $\mathrm{SL}_{2}(k)$.

In both cases, the quotient has the homotopy type of a torus whose dimension equals the rank of $k[C] \times / k^{\times}$.

Proof. We give the argument for $\mathrm{SL}_{2}$, the argument for $\mathrm{GL}_{2}$ is similar. The proof is structured as follows: in step (1), we describe a homomorphism $\tau: \mathcal{S T} \rightarrow \mathrm{SL}_{2}(k[C])$ and show in step (2) that the map $\phi_{\mathcal{L}}$ from Lemma 5.6 is $\rho$-equivariant. Steps $(3)$ to (5) deduce the claim from these facts.

(1) Recall from Definition 5.4 that $\mathcal{S T}$ was defined as the subgroup of $\mathbb{Z}^{s}$ containing the tuples $\left(2 a_{1}, \ldots, 2 a_{s}\right)$ with $\sum_{i=1}^{s} a_{i}\left[P_{i}\right]=0$ in $\operatorname{Pic}(\bar{C})$. This is a free abelian group isomorphic to $k[C]^{\times} / k^{\times}$: the condition on the sum in the Picard group implies that $\sum a_{i}\left[P_{i}\right]$ is a principal divisor $(f)$, and $f \in k[C]^{\times} / k^{\times}$. Choose an embedding $u: \mathcal{S T} \rightarrow k[C]^{\times}$which induces one such isomorphism $\mathcal{S T} \cong k[C]^{\times} / k^{\times}$ and define a homomorphism $\tau: \mathcal{S T} \rightarrow \mathrm{SL}_{2}(K)^{s}$ by sending $\left(2 a_{1}, \ldots, 2 a_{s}\right)$ to

$$
\left(M_{1}, \ldots, M_{s}\right) \cdot\left(\begin{array}{cc}
u\left(2 a_{1}, \ldots, 2 a_{s}\right) & 0 \\
0 & u\left(2 a_{1}, \ldots, 2 a_{s}\right)^{-1}
\end{array}\right) \cdot\left(M_{1}^{-1}, \ldots, M_{s}^{-1}\right) .
$$

By definition, $\tau\left(2 a_{1}, \ldots, 2 a_{s}\right)$ stabilizes the image of $\left(M_{1}, \ldots, M_{s}\right)$ in $\mathrm{SL}_{2}(k[C]) \backslash \mathfrak{P}_{C}$ if $\sum_{i=1}^{s} a_{i}\left[P_{i}\right]=0$ in $\operatorname{Pic}(\bar{C})$ : it sends the vector bundle $\mathcal{L} \oplus \mathcal{L}^{-1}$ to $\left(\mathcal{L} \otimes \mathcal{O}\left(-2 a_{1} P_{1}\right) \otimes\right.$ $\left.\cdots \otimes \mathcal{O}\left(-2 a_{s} P_{s}\right)\right) \oplus \mathcal{L}^{-1}$, but these are both isomorphic under the above condition. In this case, $\tau\left(2 a_{1}, \ldots, 2 a_{s}\right) \in \mathrm{SL}_{2}(k[C])$ because it stabilizes the double coset $\left(M_{1}, \ldots, M_{s}\right)$ in $\mathrm{SL}_{2}(k[C]) \backslash \prod \mathrm{GL}_{2}(K) /\left(\prod \mathrm{GL}_{2}\left(\mathcal{O}_{P_{i}}\right) \cdot K^{\times}\right)$. We see that $\tau: \mathcal{S T} \rightarrow$ $\mathrm{SL}_{2}(K)^{s}$ factors through a homomorphism $\mathcal{S T} \rightarrow \mathrm{SL}_{2}(k[C])$ which we will still denote $\tau$.

(2) We show that the embedding $\phi_{\mathcal{L}}$ from Lemma 5.6 is $\tau$-equivariant. The element $\left(2 a_{1}, \ldots, 2 a_{s}\right)$ acts on the model complex $\mathcal{Z}^{s}$ by the evident translation. The element $\tau\left(2 a_{1}, \ldots, 2 a_{2}\right)$ sends $\left(M_{1}, \ldots, M_{s}\right) \in \mathfrak{A}_{\mathcal{L}}$ to

$$
\left(M_{1} \cdot\left(\begin{array}{cc}
\pi_{1}^{a_{1}} & 0 \\
0 & \pi_{1}^{-a_{1}}
\end{array}\right), \cdots, M_{s} \cdot\left(\begin{array}{cc}
\pi_{s}^{a_{s}} & 0 \\
0 & \pi_{s}^{-a_{s}}
\end{array}\right)\right)
$$

which is also the translation by $\left(2 a_{1}, \ldots, 2 a_{s}\right)$ on $\mathfrak{A}_{\mathcal{L}}$.

(3) From (1) and (2) above we obtain a map of quotient cell complexes

$$
\overline{\phi_{\mathcal{L}}}: \mathcal{Z}^{s} / \mathcal{S T} \rightarrow \mathrm{SL}_{2}(k[C]) \backslash \mathfrak{P}_{C}
$$

We first show that this map is surjective onto the $\mathcal{L}$-component of $\mathrm{SL}_{2}(k[C]) \backslash \mathfrak{P}_{C}$. By Lemma 5.6 (2), we know that the 0-cells of the image are exactly the equivalence $($ rel $\partial C)$ classes of rank two bundles of the form $\left(\mathcal{L} \otimes \mathcal{O}\left(a_{1} P_{1}\right) \otimes \cdots \otimes \mathcal{O}\left(a_{s} P_{s}\right)\right) \oplus \mathcal{L}^{-1}$. For each $\left(a_{1}, \ldots, a_{s}\right) \in \mathcal{Z}^{s}$, the map $\overline{\phi_{\mathcal{L}}}$ induces an isomorphism from the star of $\left(a_{1}, \ldots, a_{s}\right)$ in $\mathcal{Z}^{s}$ to the star of $\overline{\phi_{\mathcal{L}}}$ in $\mathrm{SL}_{2}(k[C]) \backslash \mathfrak{P}_{C}$, by Proposition 4.5. Therefore, the map $\overline{\phi_{\mathcal{L}}}$ is a surjection onto the $\mathcal{L}$-component of $\mathrm{SL}_{2}(k[C]) \backslash \mathfrak{P}_{C}$. 
(4) We show that the map $\overline{\phi_{\mathcal{L}}}$ is injective. First, we consider 0-cells. Let $\left(a_{1}, \ldots, a_{s}\right)$ and $\left(b_{1}, \ldots, b_{s}\right)$ be two vertices of $\mathcal{Z}^{s}$. The vector bundles associated to these two vertices are

$$
\left(\mathcal{L} \otimes \mathcal{O}\left(a_{1} P_{1}\right) \otimes \cdots \otimes \mathcal{O}\left(a_{s} P_{s}\right)\right) \oplus \mathcal{L}^{-1} \text { and }\left(\mathcal{L} \otimes \mathcal{O}\left(b_{1} P_{1}\right) \otimes \cdots \otimes \mathcal{O}\left(b_{s} P_{s}\right)\right) \oplus \mathcal{L}^{-1} .
$$

These vector bundles are isomorphic precisely when $\sum\left(a_{i}-b_{i}\right) P_{i}=0$ in $\operatorname{Pic}(\bar{C})$. But then the two original vertices are $\mathcal{S} \mathcal{T}$-conjugate. This shows injectivity on 0 -cells. Injectivity for $n$-cells now follows from injectivity of 0 -cells and the fact - established in Proposition 4.5 and used in step (3) above - that $\overline{\phi_{\mathcal{L}}}$ induces isomorphisms on stars.

(5) Finally, the stabilizer statements follow from Proposition 2.5 and the fact that all vector bundles are split with non-isomorphic direct summands. It is also clear that the quotient modulo the group of translations has the homotopy type of a torus of the rank claimed.

Proposition 5.8. Let $k$ be an algebraically closed field, let $\bar{C}$ be a smooth projective curve and denote $C=\bar{C} \backslash\left\{P_{1}, \ldots, P_{s}\right\}$. Let $\mathcal{L}$ be a line bundle on $\bar{C}$ such that $\left.\left.\mathcal{L}\right|_{C} \cong \mathcal{L}^{-1}\right|_{C}$.

(1) The connected component of $\mathrm{GL}_{2}(k[C]) \backslash \mathfrak{P}_{C}$ containing the vector bundle $\mathcal{L} \oplus \mathcal{L}$ is isomorphic to $\mathcal{Z}^{s} / \mathcal{N}$. The image of $(0, \ldots, 0)$ is stabilized by $\mathrm{GL}_{2}(k) \times_{k} \times k[C]^{\times}$. The stabilizers of all other cells are of the form $\left(k^{n} \rtimes\right.$ $\left.\left(k^{\times}\right)^{2}\right) \times_{k^{\times}} k[C]^{\times}$for some $n$ depending on the cell, i.e., up to the unipotent radical all the other cells have stabilizer $\mathrm{T} \times_{k} \times k[C]^{\times}$where $\mathrm{T}$ is a maximal torus in $\mathrm{GL}_{2}(k)$.

(2) The connected component of $\mathrm{SL}_{2}(k[C]) \backslash \mathfrak{P}_{C}$ containing the vector bundle $\mathcal{L} \oplus \mathcal{L}$ is isomorphic to $\mathcal{Z}^{s} / \mathcal{S N}$. There are $2^{\text {rk } \mathcal{T}}$ points with stabilizer $\mathrm{SL}_{2}(k)$, they are the $\mathcal{S} \mathcal{T}$-conjugacy classes of $\mathcal{T}$-translates of $(0, \ldots, 0)$. The stabilizers of all other cells are of the form $k^{n} \rtimes k^{\times}$for some $n$ depending on the cell, i.e., up to the unipotent radical all the other cells have stabilizer a maximal torus in $\mathrm{SL}_{2}(k)$.

Proof. We give the argument for $\mathrm{SL}_{2}$, the argument for $\mathrm{GL}_{2}$ is similar but easier. The proof structure is similar to the proof of Proposition 5.7.

(1) We construct a homomorphism $\nu: \mathcal{S N} \rightarrow \mathrm{SL}_{2}(k[C])$ as follows: on the subgroup $\mathcal{S} \mathcal{T}$, the morphism $\nu$ agrees with $\tau$. Now for $\sum_{i=1}^{s} a_{i}\left[P_{i}\right]=0$ in $\operatorname{Pic}(\bar{C})$ and $r\left(a_{1}, \ldots, a_{s}\right)$ the point-reflection with center $\left(a_{1}, \ldots, a_{s}\right)$, the map $\nu$ is defined to be

$$
\left(M_{1}, \ldots, M_{s}\right) \cdot\left(\begin{array}{cc}
0 & u\left(a_{1}, \ldots, a_{s}\right) \\
-u\left(a_{1}, \ldots, a_{s}\right)^{-1} & 0
\end{array}\right) \cdot\left(M_{1}^{-1}, \ldots, M_{s}^{-1}\right) .
$$

Then $\nu\left(r\left(a_{1}, \ldots, a_{s}\right)\right)$ again stabilizes the image of

$$
\left(M_{1} \cdot\left(\begin{array}{cc}
1 & 0 \\
0 & \pi_{1}^{a_{1}}
\end{array}\right), \ldots, M_{s} \cdot\left(\begin{array}{cc}
1 & 0 \\
0 & \pi_{s}^{a_{s}}
\end{array}\right)\right)
$$

in $\mathrm{SL}_{2}(k[C]) \backslash \mathfrak{P}_{C}$ under the condition $\sum_{i=1}^{s} a_{i}\left[P_{i}\right]=0$. We see that $\nu: \mathcal{S N} \rightarrow$ $\mathrm{SL}_{2}(K)^{s}$ factors through a homomorphism $\mathcal{S N} \rightarrow \mathrm{SL}_{2}(k[C])$ which we will still denote $\nu$.

(2) We show that the embedding $\phi_{\mathcal{L}}$ from Lemma 5.6 is $\nu$-equivariant. Equivariance on the subgroup $\mathcal{S T}$ follows from (2) in the proof of Proposition 5.7. We need to show that $\nu\left(r\left(a_{1}, \ldots, a_{s}\right)\right)$ is again a point-reflection. The definition of $\nu\left(r\left(a_{1}, \ldots, a_{s}\right)\right)$ shows that it sends the coset

$$
\left(M_{1} \cdot\left(\begin{array}{cc}
1 & 0 \\
0 & \pi_{1}^{b_{1}}
\end{array}\right), \ldots, M_{s} \cdot\left(\begin{array}{cc}
1 & 0 \\
0 & \pi_{s}^{b_{s}}
\end{array}\right)\right)
$$


to the coset

$$
\left(M_{1} \cdot\left(\begin{array}{cc}
\pi_{1}^{b_{1}-2 a_{1}} & 0 \\
0 & 1
\end{array}\right), \ldots, M_{s} \cdot\left(\begin{array}{cc}
\pi_{s}^{b_{s}-2 a_{s}} & 0 \\
0 & 1
\end{array}\right)\right)
$$

This proves the claim.

(3) The equivariance from (1) and (2) shows that we obtain a map of quotient cell complexes

$$
\overline{\phi_{\mathcal{L}}}: \mathcal{Z}^{s} / \mathcal{S N} \rightarrow \mathrm{SL}_{2}(k[C]) \backslash \mathfrak{P}_{C}
$$

Surjectivity of $\overline{\phi_{\mathcal{L}}}$ onto the $\mathcal{L}$-component of $\mathrm{SL}_{2}(k[C]) \backslash \mathfrak{P}_{C}$ follows as in the proof of Proposition 5.7 from the results in Lemma 5.6 and Proposition 4.5.

(4) For injectivity on 0 -cells, let $\left(a_{1}, \ldots, a_{s}\right)$ and $\left(b_{1}, \ldots, b_{s}\right)$ be vertices of $\mathcal{Z}^{s}$ which induce isomorphic vector bundles

$$
\left(\mathcal{L} \otimes \mathcal{O}\left(a_{1} P_{1}\right) \otimes \cdots \otimes \mathcal{O}\left(a_{s} P_{s}\right)\right) \oplus \mathcal{L} \text { and }\left(\mathcal{L} \otimes \mathcal{O}\left(b_{1} P_{1}\right) \otimes \cdots \otimes \mathcal{O}\left(b_{s} P_{s}\right)\right) \oplus \mathcal{L} .
$$

The isomorphism of vector bundles implies that the line bundles $\mathcal{O}\left(a_{1} P_{1}\right) \otimes \cdots \otimes$ $\mathcal{P}\left(a_{s} P_{s}\right)$ and $\mathcal{O}\left(b_{1} P_{1}\right) \otimes \cdots \otimes \mathcal{P}\left(b_{s} P_{s}\right)$ are either isomorphic or inverses. The case of isomorphisms is the one handled in (4) of the proof of Proposition 5.7. If they are inverses, then their tensor product is a trivial bundle, and the corresponding vector bundles are related by a point reflection with center corresponding to the vector bundle $\left(\mathcal{L} \otimes \mathcal{O}\left(\left(a_{1}+b_{1}\right) P_{1}\right) \otimes \cdots \otimes \mathcal{O}\left(\left(a_{s}+b_{s}\right) P_{s}\right)\right) \oplus \mathcal{L}$. The tuples $\left(a_{1}, \ldots, a_{s}\right)$ and $\left(b_{1}, \ldots, b_{s}\right)$ are then $\mathcal{S} \mathcal{N}$-conjugate, and we get injectivity on 0 -cells. Injectivity for $n$-cells then follows again from Proposition 4.5.

(5) Finally, the stabilizer statements follow from Proposition 2.5 and the fact that all vector bundles are split with non-isomorphic direct summands.

5.3. Functoriality. From Proposition 3.12, we know that morphisms of curves $f: \bar{C} \rightarrow \bar{D}$ induce morphisms of parabolic subcomplexes and parabolic quotients. Now that we have precise knowledge of the structure of the parabolic quotients, we can also describe precisely the effect of the induced morphisms:

Proposition 5.9. Let $k$ be an algebraically closed field. Let $f: \bar{D} \rightarrow \bar{C}$ be a finite morphism of smooth projective curves over $k$, let $P_{1}, \ldots, P_{s}$ be points on $\bar{C}$, and let $Q_{1}, \ldots, Q_{t}$ be points on $\bar{D}$ not in the preimage of the $P_{i}$. Set $C=\bar{C} \backslash\left\{P_{1}, \ldots, P_{s}\right\}$ and $D=\bar{D} \backslash\left(\left\{f^{-1}\left(\left\{P_{1}, \ldots, P_{s}\right\}\right) \cup\left\{Q_{1}, \ldots, Q_{t}\right\}\right)\right.$.

Then we have the following assertions for the induced morphism $f^{*}: \mathfrak{P}_{C} \rightarrow \mathfrak{P}_{D}$ of Proposition 3.12:

(1) The composition

$$
\mathcal{K}(C) \rightarrow \pi_{0}\left(\mathrm{GL}_{2}(k[C]) \backslash \mathfrak{P}_{C}\right) \stackrel{\pi_{0}\left(f^{*}\right)}{\longrightarrow} \pi_{0}\left(\mathrm{GL}_{2}(k[D]) \backslash \mathfrak{P}_{D}\right) \rightarrow \mathcal{K}(D)
$$

is induced from pullback of line bundles $f^{*}: \operatorname{Pic}(C) \rightarrow \operatorname{Pic}(D)$, where the first and last map are the bijections of Lemma 5.2.

(2) The morphism from a given component to the image component can be described on model complexes as follows: the map $\mathcal{Z}^{s} \rightarrow \mathcal{Z}^{\tilde{s}+t}$ maps the $i$-th coordinate diagonally to $f^{-1}\left(P_{i}\right)$ many coordinates of $\mathcal{Z}^{\tilde{s}+t}$.

(3) The composition $k[C]^{\times} / k^{\times} \cong \mathcal{T}_{C} \rightarrow \mathcal{T}_{D} \cong k[D]^{\times} / k^{\times}$is induced from the natural map $f^{*}: k[C] \rightarrow k[D]$ on function rings.

Proof. (1) is clear and follows because $f^{*}$ on the vertices of the quotients is given by pullback of vector bundles.

(2) the map on model complexes is the same one as on the standard apartment of the building, and hence the same as the one described in Proposition 3.12.

(3) the map is induced from the inclusion of the units in the function fields and the conjugation action on certain apartments. Therefore, it is the natural map. 


\section{EqUiVARIANT HOMOLOGY OF THE PARABOLIC SUBCOMPLEX}

In this section, we will use the description of the quotients $\Gamma \backslash \mathfrak{P}_{C}$ to compute the $\Gamma$-equivariant homology of $\mathfrak{P}_{C}$, for $\Gamma$ one of the linear groups $(\mathrm{P}) \mathrm{GL}_{2}(k[C])$ and $(\mathrm{P}) \mathrm{SL}_{2}(k[C])$. The main results are Proposition 6.1 and Proposition 6.3 for the two types of connected components: the components for non-2-torsion line bundles contribute the homology of $k[C]^{\times}$while the components for 2-torsion line bundles contribute groups related to a normalizer of $k[C]^{\times}$. Using these explicit descriptions, we can also completely describe the morphisms on parabolic homology induced from any quasi-finite morphism of affine curves $f: D \rightarrow C$.

6.1. Recollection on equivariant homology. We shortly recall equivariant homology and the Borel isotropy spectral sequence for computing it, cf. Bro94, Section VII] or [Knu01, Appendix A].

A $G$-complex is a CW-complex $X$ together with an action $G \times X \rightarrow X$ which is cellular. If $X$ is a $G$-complex, then there is an action of $G$ on the cellular chain complex $\mathrm{C} \bullet(X)$, and the corresponding homology groups

$$
\mathrm{H}_{\bullet}^{G}(X, M):=\mathrm{H}_{\bullet}\left(G, \mathrm{C} \bullet(X) \otimes_{\mathbb{Z}[G]} M\right)
$$

are called equivariant homology groups of $(G, X)$. If $X$ is contractible (or even just acyclic), then the equivariant homology of $X$ is group homology, i.e. $\mathrm{H}_{\bullet}^{G}(X, M) \cong$ H. $(G, M)$.

For a $G$-complex $X$, the $G$-equivariant homology of $X$ can be computed by means of the following spectral sequence, which arises from the stupid filtration of $\mathrm{C} \bullet(X) \otimes_{\mathbb{Z}[G]} M$ :

$$
E_{p, q}^{1}=\bigoplus_{\sigma \in \Sigma_{p}} \mathrm{H}_{q}\left(G_{\sigma}, M_{\sigma}\right) \Rightarrow \mathrm{H}_{p+q}^{G}(X, M) .
$$

In the above, $\Sigma_{p}$ denotes a set of representatives for the $G$-action on $\mathrm{C}_{p}(X)$. The module $M_{\sigma}$ is the coefficient $G$-module $M$ twisted by the orientation character $\chi_{\sigma}: G_{\sigma} \rightarrow \mathbb{Z} / 2$ of the stabilizer group $G_{\sigma}$. In the special case where each cell stabilizer fixes the cell pointwise, the orientation character is trivial. This can always be achieved by a suitable subdivision of cells. The first differential is induced from the boundary map of the complex $X$ and inclusions of stabilizers, cf. Bro94, VII.8]:

$$
\left.d^{1}\right|_{\mathrm{H}_{q}\left(G_{\sigma}, M_{\sigma}\right)}: \mathrm{H}_{q}\left(G_{\sigma}, M_{\sigma}\right) \mapsto \bigoplus_{\tau \subseteq \sigma} \mathrm{H}_{q}\left(G_{\tau}, M_{\tau}\right)
$$

In the following we consider the groups $(\mathrm{P}) \mathrm{GL}_{2}(k[C])$ resp. $(\mathrm{P}) \mathrm{SL}_{2}(k[C])$ acting on the cubical complex $\mathfrak{P}_{C}$.

6.2. Components of torus type. We first evaluate the equivariant homology of the components corresponding to line bundles $\mathcal{L}$ with $\mathcal{L} \neq \mathcal{L}^{-1}$. These components are called "of torus type" for two reasons. On the one hand, the quotient has the homotopy type of a torus. On the other hand, the equivariant homology is mainly influenced by the intersection of $\mathrm{GL}_{2}(k[C])$ with a maximal torus in $\mathrm{GL}_{2}(K)^{s}$.

Proposition 6.1. Let $k$ be an algebraically closed field, let $\bar{C}$ be a smooth projective curve and denote $C=\bar{C} \backslash\left\{P_{1}, \ldots, P_{s}\right\}$. Let $\mathcal{L}$ be a line bundle on $\bar{C}$ such that $\left.\mathcal{L}\right|_{C} \neq\left.\mathcal{L}\right|_{C} ^{-1}$ and denote by $\mathfrak{P}_{C}(\mathcal{L})$ the connected component of $\mathfrak{P}_{C}$ corresponding to $\mathcal{L}$.

(1) The $\mathcal{T}$-equivariant inclusion $\mathcal{Z}^{s} \rightarrow \mathfrak{P}_{C}$ from Proposition 5.7 induces isomorphisms on homology

$$
\begin{aligned}
\mathrm{H}_{\bullet}^{\mathrm{GL}_{2}(k[C])}\left(\mathfrak{P}_{C}(\mathcal{L}), \mathbb{Z}\right) & \cong \mathrm{H}_{\bullet}\left(\left(k[C]^{\times}\right)^{2}, \mathbb{Z}\right) \\
\mathrm{H}_{\bullet}^{\mathrm{PGL}_{2}(k[C])}\left(\mathfrak{P}_{C}(\mathcal{L}), \mathbb{Z}\right) & \cong \mathrm{H}_{\bullet}\left(k[C]^{\times}, \mathbb{Z}\right)
\end{aligned}
$$


(2) The $\mathcal{S} \mathcal{T}$-equivariant inclusion $\mathcal{Z}^{s} \rightarrow \mathfrak{P}_{C}$ from Proposition 5.7 induces an isomorphism on homology

$$
\mathrm{H}_{\bullet}^{(\mathrm{P}) \mathrm{SL}_{2}(k[C])}\left(\mathfrak{P}_{C}(\mathcal{L}), \mathbb{Z}\right) \cong \mathrm{H}_{\bullet}\left(k[C]^{\times}, \mathbb{Z}\right) .
$$

Proof. We give the argument for $\mathrm{SL}_{2}$, the argument for $\mathrm{GL}_{2}$ is similar. Recall from Proposition 5.7 that we have an equivariant map $\mathcal{Z}^{s} \rightarrow \mathfrak{P}_{C}(\mathcal{L})$. From the proof of Proposition 5.7, we see that the homomorphism $\tau: \mathcal{T} \rightarrow \mathrm{SL}_{2}(k[C])$ extends to a homomorphism $\tilde{\tau}: k[C]^{\times} \rightarrow \mathrm{SL}_{2}(k[C])$. Using the stabilizer computations of Proposition 5.7, the map $\mathcal{Z}^{s} \rightarrow \mathfrak{P}_{C}(\mathcal{L})$ is still equivariant with respect to $\tilde{\tau}$, where the action of $k[C]^{\times}$on $\mathcal{Z}^{s}$ is the one factoring through $k[C]^{\times} \rightarrow k[C]^{\times} / k^{\times} \cong \mathcal{T}$.

The equivariant map above induces a morphism of isotropy spectral sequences computing the induced morphism on homology

$$
\mathrm{H}_{\bullet}^{k[C]^{\times}}\left(\mathcal{Z}^{s}, \mathbb{Z}\right) \rightarrow \mathrm{H}_{\bullet}^{\mathrm{SL}_{2}(k[C])}\left(\mathfrak{P}_{C}(\mathcal{L}), \mathbb{Z}\right) .
$$

By Proposition 5.7, the map $\mathcal{Z}^{s} \rightarrow \mathfrak{P}_{C}(\mathcal{L})$ induces an isomorphism of quotient cell complexes. Any cell of $\mathcal{Z}^{s}$ is stabilized by $k^{\times}$, and the cells of $\mathfrak{P}_{C}(\mathcal{L})$ are stabilized by groups of the form $k^{n} \rtimes k^{\times}$where the size of the unipotent group depends on the cell. Note that the action of $k^{\times}$on $k^{n}$ in the above semi-direct product is induced from an embedding into a Borel subgroup of $\mathrm{GL}_{2}(K)$. For any cell $\sigma$ in $\mathcal{Z}^{s}$, the composition

$$
k^{\times} \cong \operatorname{Stab}\left(\sigma ; k[C]^{\times}\right) \rightarrow \operatorname{Stab}\left(\sigma ; \mathrm{SL}_{2}(k[C])\right) \cong k^{n} \rtimes k^{\times} \stackrel{p}{\longrightarrow} k^{\times}
$$

is the identity. By [Knu01, Theorem 4.6.2], the inclusion

$$
\operatorname{Stab}\left(\sigma ; k[C]^{\times}\right) \rightarrow \operatorname{Stab}\left(\sigma ; \mathrm{SL}_{2}(k[C])\right)
$$

induces an isomorphism in group homology. The morphism of equivariant spectral sequences is then an isomorphism on the $E^{1}$-page, and hence induces an isomorphism on equivariant homology.

The claim now follows easily, we simply compute $\mathrm{H}_{\bullet}^{k[C]^{\times}}\left(\mathcal{Z}^{s}, \mathbb{Z}\right)$ : the model complex $\mathcal{Z}^{s}$ is contractible, and the action of $k[C]^{\times}$factors as a free action of $k[C]^{\times} / k^{\times}$ and a trivial action of $k^{\times}$. In particular, we have an isomorphism

$$
\mathrm{H}_{\bullet}^{k[C]^{\times}}\left(\mathcal{Z}^{s}, \mathbb{Z}\right) \cong \mathrm{H}_{\bullet}^{k^{\times}}\left(\left(k[C]^{\times} / k^{\times}\right) \backslash \mathcal{Z}^{s}, \mathbb{Z}\right) .
$$

Note that the homology of the quotient $X=\left(k[C]^{\times} / k^{\times}\right) \backslash \mathcal{Z}^{s}$ is the homology of the free abelian group $k[C]^{\times} / k^{\times}$, in particular it is torsion-free. The $k^{\times}$-equivariant homology of $X$ is defined as the homology of the Borel construction $k^{\times} \backslash\left(E k^{\times} \times X\right)$. Since $k^{\times}$acts trivially on $X$, this quotient can be identified as $B k^{\times} \times X$. In particular, applying the Künneth formula, we see that the $k^{\times}$-equivariant homology of $X$ is the homology of the group $k[C]^{\times}$.

6.3. Components of normalizer type, case $\mathrm{SL}_{2}$. We next study the homology of the components corresponding to 2-torsion line bundles. These components are called "of normalizer type" since the quotient is (away from 2) a classifying space for the intersection of $\mathrm{SL}_{2}(k[C])$ with the normalizer of a maximal torus in $\mathrm{SL}_{2}(k(C))$. The argument in this case is more involved since there are points which have stabilizer $\mathrm{SL}_{2}(k)$, which is too large to just come from the normalizer of a maximal torus.

We recall from Section 5 what we know about the global structure of those normalizer type components. We fix a line bundle $\mathcal{L}$ with $\mathcal{L} \cong \mathcal{L}^{-1}$ and denote by $\mathfrak{P}_{C}(\mathcal{L})$ the connected component of the parabolic subcomplex $\mathfrak{P}_{C}$ containing the bundle $\mathcal{L} \oplus \mathcal{L}$. Combining Lemma 5.6 and Proposition 5.8, there is a map $\phi_{\mathcal{L}}: \mathcal{Z}^{s} \rightarrow \mathfrak{A}_{\mathcal{L}} \subset \mathfrak{P}_{C}(\mathcal{L})$ and a homomorphism $\nu: \mathcal{S N} \rightarrow \mathrm{SL}_{2}(k[C])$ such that $\phi_{\mathcal{L}}$ is $\nu$-equivariant and induces an isomorphism of quotient complexes $\mathcal{Z}^{s} / \mathcal{S N} \cong$ $\mathrm{SL}_{2}(k[C]) \backslash \mathfrak{P}_{C}(\mathcal{L})$. From (1) in the proof of Proposition 5.8, we see that $\nu: \mathcal{S N} \rightarrow$ 
$\mathrm{SL}_{2}(k[C])$ can be extended to a group homomorphism $\tilde{\nu}: \tilde{\mathcal{S N}} \rightarrow \mathrm{SL}_{2}(k[C])$, where $\tilde{S N}$ is the group of determinant 1 monomial $(2 \times 2)$-matrices with entries in $k[C]$, i.e.,

$$
\tilde{\mathcal{S N}}=\left\{\left(\begin{array}{cc}
0 & u \\
-u^{-1} & 0
\end{array}\right) \mid u \in k[C]^{\times}\right\} \cup\left\{\left(\begin{array}{cc}
u & 0 \\
0 & u^{-1}
\end{array}\right) \mid u \in k[C]^{\times}\right\} .
$$

The group $\tilde{\mathcal{S N}}$ sits in an extension

$$
1 \rightarrow k^{\times} \rightarrow \tilde{\mathcal{S N}} \rightarrow \mathcal{S N} \rightarrow 1
$$

the homomorphism $\tilde{\nu}$ is the natural embedding followed by conjugation as in (1) of Proposition 5.8, and $\nu$ is the composition of $\tilde{\nu}$ with a choice of section $\mathcal{S N} \rightarrow \tilde{\mathcal{S N}}$. Of course, the subgroup $k^{\times}$above acts trivially on $\mathcal{Z}^{s}$ and hence does not contribute to the quotient; its relevance for the isotropy spectral sequence comes from the contribution to the stabilizer subgroups.

Lemma 6.2. Consider the above action of $\tilde{\mathcal{S N}}$ on $\mathcal{Z}^{s}$. With $\mathbb{Z}[1 / 2]$-coefficients, the isotropy spectral sequence for the $\tilde{\mathcal{S N}}$-complex $\mathcal{Z}^{s}$ degenerates at the $E^{2}$-page and produces an isomorphism

$$
\mathrm{H}_{\bullet}^{\tilde{\mathcal{N}}}\left(\mathcal{Z}^{s}, \mathbb{Z}[1 / 2]\right) \cong \mathrm{H}_{\bullet}(\tilde{\mathcal{S N}}, \mathbb{Z}[1 / 2])
$$

Proof. (1) We first describe the stabilizer subgroups to infer some information on the page $E_{p, q}^{1}=\bigoplus_{\sigma \in \Sigma_{p}} \mathrm{H}_{q}\left(\tilde{\mathcal{S N}}_{\sigma}, \mathbb{Z}[1 / 2]\right)$. As noted above, the subgroup $k^{\times}$(embedded as constant diagonal matrices) acts trivially, hence every stabilizer contains at least a copy of $k^{\times}$. The group $\mathcal{S N}$ sits in an extension

$$
0 \rightarrow \mathcal{S T} \rightarrow \mathcal{S N} \rightarrow k[C]^{\times} /\left(k[C]^{\times}\right)^{2} \rightarrow 1
$$

The subgroup $\mathcal{S} \mathcal{T}$ acts via translations on $\mathcal{Z}^{s}$, hence acts without fixed points. The square classes in the quotient act as point inversions with suitable centers, as described in Definition 5.4 Therefore, the vertices $\left(a_{1}, \ldots, a_{s}\right)$ with $\sum a_{i}\left[P_{i}\right]=0$ have stabilizers $k^{\times} \rtimes \mathbb{Z} / 2$ where the $\mathbb{Z} / 2$-factor acts via inversion on $k^{\times}$. All other vertices have $k^{\times}$as stabilizer. Because the action of the $\mathbb{Z} / 2$-factors on $\mathcal{Z}^{s}$ is via point inversions, the higher simplices also all have $k^{\times}$as stabilizer.

(2) Note that the identity on $\mathcal{Z}^{s}$ is equivariant for the inclusion $\tilde{\mathcal{S T}} \hookrightarrow \tilde{\mathcal{S N}}$, where $\tilde{\mathcal{S T}}$ is the group of determinant diagonal $(2 \times 2)$-matrices with entries in $k[C]$. The inclusion therefore induces a morphism of isotropy spectral sequences converging to the morphism

$$
\mathrm{H}_{\bullet}^{\tilde{\mathcal{S T}}}\left(\mathcal{Z}^{s}, \mathbb{Z}[1 / 2]\right) \rightarrow \mathrm{H}_{\bullet}^{\tilde{\mathcal{N}}}\left(\mathcal{Z}^{s}, \mathbb{Z}[1 / 2]\right) .
$$

The first group is the one discussed in the torus case above, and the isotropy spectral sequence for $\tilde{\mathcal{S T}}$-equivariant homology of $\mathcal{Z}^{s}$ degenerates at the $E^{2}$-page. In particular, the restrictions of the differentials to the homology of the stabilizers in $\tilde{\mathcal{S T}}$ is trivial.

(3) Now we note that the quotient map $\mathcal{Z}^{s} \rightarrow \mathcal{Z}^{s} / \tilde{\mathcal{S T}}$ is equivariant for the quotient homomorphism

$$
\tilde{\mathcal{S N}} \rightarrow \tilde{\mathcal{S N}} / \tilde{\mathcal{S T}} \cong k[C]^{\times} /\left(k[C]^{\times}\right)^{2} .
$$

We consider the $k[C]^{\times} /\left(k[C]^{\times}\right)^{2}$-equivariant homology of the quotient $\mathcal{Z}^{s} / \tilde{\mathcal{S T}}$. Obviously, the stabilizers in this case are only 2 -torsion. Therefore, the only non-trivial terms of the spectral sequence for $k[C]^{\times} /\left(k[C]^{\times}\right)^{2}$-equivariant homology of $\mathcal{Z}^{s} / \tilde{\mathcal{S T}}$ with $\mathbb{Z}[1 / 2]$-coefficients are concentrated in the $q=0$-column. Hence the spectral sequence degenerates at the $E^{2}$-term, and all further differentials are trivial.

(4) Combining points (2) and (3) above, we find that all differentials $d_{r}, r \geq 2$, of the isotropy spectral sequence computing $\tilde{\mathcal{S N}}$-equivariant homology of $\mathcal{Z}^{s}$ have to be trivial: the quotient map from (3) induces an isomorphism on the $q=0$ 
part, and everything else is detected on the subgroup $\tilde{\mathcal{S T}}$ from (2). The claim follows.

Proposition 6.3. Let $k$ be an algebraically closed field, let $\bar{C}$ be a smooth projective curve and denote $C=\bar{C} \backslash\left\{P_{1}, \ldots, P_{s}\right\}$. Let $\mathcal{L}$ be a line bundle on $\bar{C}$ which is 2torsion in the Picard group of $C$, and denote by $\mathfrak{P}_{C}(\mathcal{L})$ the corresponding connected component of the parabolic subcomplex, cf. Proposition 5.8.

Denote $\tilde{\nu}: \tilde{\mathcal{S N}} \rightarrow \mathrm{SL}_{2}(k[C])$ the group homomorphism discussed above. The $\tilde{\nu}$-equivariant map $\phi_{\mathcal{L}}: \mathcal{Z}^{s} \rightarrow \mathfrak{P}_{C}(\mathcal{L})$ induces a homomorphism

$$
\mathrm{H}_{\bullet}(\tilde{\mathcal{S N}}, \mathbb{Z}[1 / 2]) \cong \mathrm{H}_{\bullet}^{\tilde{\mathcal{N}}}\left(\mathcal{Z}^{s}, \mathbb{Z}[1 / 2]\right) \rightarrow \mathrm{H}_{\bullet}^{\mathrm{SL}_{2}(k[C])}\left(\mathfrak{P}_{C}(\mathcal{L}), \mathbb{Z}[1 / 2]\right)
$$

such that there is a long exact sequence of $\mathbb{Z}[1 / 2]$-modules

$$
\begin{gathered}
\quad \cdots \rightarrow \mathcal{R} \mathcal{P}_{i+1}^{1}(k) \otimes_{\mathbb{Z}} \mathbb{Z}\left[1 / 2, k[C]^{\times} /\left(k[C]^{\times}\right)^{2}\right] \rightarrow \mathrm{H}_{i}(\tilde{\mathcal{S}}, \mathbb{Z}[1 / 2]) \rightarrow \\
\rightarrow \mathrm{H}_{i}^{\mathrm{SL}_{2}(k[C])}\left(\mathfrak{P}_{C}(\mathcal{L}), \mathbb{Z}[1 / 2]\right) \rightarrow \mathcal{R} \mathcal{P}_{i}^{1}(k) \otimes_{\mathbb{Z}} \mathbb{Z}\left[1 / 2, k[C]^{\times} /\left(k[C]^{\times}\right)^{2}\right] \rightarrow \cdots
\end{gathered}
$$

where the groups $\mathcal{R P}^{1} \cdot(k)$ are the refined scissors configurations groups recalled in Section A.

Proof. (1) As before, the $\tilde{\nu}$-equivariance of the map $\phi_{\mathcal{L}}: \mathcal{Z}^{s} \rightarrow \mathfrak{P}_{C}(\mathcal{L})$ follows from Proposition 5.8 together with the remarks before Lemma 6.2. This implies the existence of an induced morphism on homology

$$
\mathrm{H}_{\bullet}^{\mathcal{S \mathcal { N }}}\left(\mathcal{Z}^{s}, \mathbb{Z}[1 / 2]\right) \rightarrow \mathrm{H}_{\bullet}^{\mathrm{SL}_{2}(k[C])}\left(\mathfrak{P}_{C}(\mathcal{L}), \mathbb{Z}[1 / 2]\right)
$$

The identification of the source with group homology of $\tilde{\mathcal{S N}}$ follows from Lemma 6.2 The $\tilde{\nu}$-equivariant map $\mathcal{Z}^{s} \hookrightarrow \mathfrak{P}_{C}(\mathcal{L})$ also induces a morphism of the corresponding isotropy spectral sequences. To prove the remaining claims, we investigate this morphism of isotropy spectral sequences, where we use the following indexing for the isotropy spectral sequence:

$$
E_{p, q}^{1}=\bigoplus_{\sigma \in \Sigma_{p}} \mathrm{H}_{q}\left(G_{\sigma}, M_{\sigma}\right) \Rightarrow \mathrm{H}_{p+q}^{G}(X, M)
$$

with differentials going $d_{p, q}^{r}: E_{p, q}^{r} \rightarrow E_{p-r, q+r-1}^{r}$. We denote $E_{p, q}^{i}(\tilde{\mathcal{S N}})$ the $(p, q)$ entry of the $i$-th page of the spectral sequence for $\tilde{\mathcal{S}}$-equivariant homology of $\mathcal{Z}^{s}$, and similarly for $E_{p, q}^{i}\left(\mathrm{SL}_{2}(k[C])\right)$.

(2) From Proposition 5.8 the map $\mathcal{Z}^{s} \rightarrow \mathfrak{P}_{C}(\mathcal{L})$ induces an isomorphism of quotient cell complexes $\mathcal{Z}^{s} / \tilde{\mathcal{S N}} \cong \mathrm{SL}_{2}(k[C]) \backslash \mathfrak{P}_{C}(\mathcal{L})$. In particular, the induced morphism $E_{p, 0}^{1}(\tilde{\mathcal{S N}}) \rightarrow E_{p, 0}^{1}\left(\mathrm{SL}_{2}(k[C])\right)$ is an isomorphism of complexes and hence induces an isomorphism on the $(q=0)$-column of the $E^{2}$-pages.

(3) Next, we look at the stabilizers. For the $\tilde{\mathcal{S N}}$-action on $\mathcal{Z}^{s}$, we discussed in the proof of Lemma 6.2 that there are $2^{\text {rk } \mathcal{S T}}$ vertices stabilized by a semi-direct product $k^{\times} \rtimes \mathbb{Z} / 2$, where the quotient is generated by the point inversion. For the action of $\mathrm{SL}_{2}(k[C])$ on $\mathfrak{P}_{C}(\mathcal{L})$, there are $2^{\text {rk } \mathcal{T}}$ vertices stabilized by $\mathrm{SL}_{2}(k)$. The map $\mathcal{Z}^{s} \rightarrow \mathfrak{P}_{C}(\mathcal{L})$ sends the former to the latter types of points, and the induced morphism on stabilizers is the inclusion of $k^{\times} \rtimes \mathbb{Z} / 2$ as the normalizer of a maximal torus in $\mathrm{SL}_{2}(k)$.

All the other cells in $\mathcal{Z}^{s}$ have stabilizer $k^{\times}$in $\tilde{\mathcal{S N}}$. By Proposition 5.8, all other cells in $\mathfrak{P}_{C}(\mathcal{L})$ have stabilizer some semi-direct product $k^{n} \rtimes k^{\times}$with the unipotent group $k^{n}$ depending on the cell. As in the torus case, the composition

$$
k^{\times} \cong \tilde{\mathcal{N}}_{\sigma} \stackrel{\iota}{\longrightarrow}\left(\mathrm{SL}_{2}(k[C])\right)_{\sigma} \cong k^{n} \rtimes k^{\times} \stackrel{p}{\longrightarrow} k^{\times}
$$

is the identity for any such cell $\sigma$ in $\mathcal{Z}^{s}$. By [Knu01, Theorem 4.6.2], the inclusion $\tilde{\mathcal{N}}_{\sigma} \hookrightarrow\left(\mathrm{SL}_{2}(k[C])\right)_{\sigma}$ induces an isomorphism in group homology. In particular, the map $\mathcal{Z}^{s} \rightarrow \mathfrak{P}_{C}(\mathcal{L})$ induces an isomorphism on the $(p \geq 1)$-part of the $E^{1}$-terms: 
the homology of the stabilizers changes only for the inclusion $k^{\times} \rtimes \mathbb{Z} / 2 \hookrightarrow \mathrm{SL}_{2}(k)$ of the normalizer of a maximal torus and this happens for special 0-cells only.

(4) From (3) above, we see that the differentials $d_{p \geq 2, \bullet}^{1}$ agree and only the differential $d_{1, \bullet}^{1}: \bigoplus_{\sigma \in \Sigma_{1}} \mathrm{H}_{\bullet}\left(G_{\sigma}\right) \rightarrow \bigoplus_{\sigma \in \Sigma_{0}} \mathrm{H}_{\bullet}\left(G_{\sigma}\right)$ differs for the two spectral sequences associated to $\tilde{\mathcal{S N}} \backslash \mathcal{Z}^{s}$ and $\mathrm{SL}_{2}(k[C]) \backslash \mathfrak{P}_{C}(\mathcal{L})$. Note that even this differential is the composition of the differential $d_{1, \bullet}^{1}(\tilde{\mathcal{S N}})$ with the map $\mathrm{H}_{\bullet}(\mathrm{N}(k)) \rightarrow \mathrm{H}_{\bullet}\left(\mathrm{SL}_{2}(k)\right)$. In particular, the induced morphisms $E_{p, q}^{2}(\tilde{\mathcal{S N}}) \rightarrow E_{p, q}^{2}\left(\mathrm{SL}_{2}(k[C])\right)$ are isomorphisms for all $p \geq 2$.

Moreover, one copy of the long exact seqeuence of Proposition A.10 at every of the special vertices combine to the following long exact sequence

$$
\begin{aligned}
\cdots \rightarrow \mathcal{R} \mathcal{P}_{i+1}^{1}(k) \otimes_{\mathbb{Z}} \mathbb{Z}\left[1 / 2, k[C]^{\times} /\left(k[C]^{\times}\right)^{2}\right] & \rightarrow E_{0, i}^{1}(\tilde{\mathcal{S N}}) \rightarrow \\
& \rightarrow E_{0, i}^{1}\left(\mathrm{SL}_{2}(k[C])\right) \rightarrow \mathcal{R} \mathcal{P}_{i}^{1}(k) \otimes_{\mathbb{Z}} \mathbb{Z}\left[1 / 2, k[C]^{\times} /\left(k[C]^{\times}\right)^{2}\right] \rightarrow \cdots
\end{aligned}
$$

(5) In the next step, we want to establish an exact sequence describing the morphism of $E^{2}$-terms. First note that the map

$$
E_{0, i}^{1}\left(\mathrm{SL}_{2}(k[C])\right) \rightarrow \mathcal{R} \mathcal{P}_{i}^{1}(k) \otimes_{\mathbb{Z}} \mathbb{Z}\left[1 / 2, k[C]^{\times} /\left(k[C]^{\times}\right)^{2}\right]
$$

induces a natural map

$$
E_{0, i}^{2}\left(\mathrm{SL}_{2}(k[C])\right) \rightarrow \mathcal{R} \mathcal{P}_{i}^{1}(k) \otimes_{\mathbb{Z}} \mathbb{Z}\left[1 / 2, k[C]^{\times} /\left(k[C]^{\times}\right)^{2}\right]
$$

because the differential $d^{1}: E_{1, i}^{1} \rightarrow E_{0, i}^{1}\left(\mathrm{SL}_{2}(k[C])\right)$ factors through $E_{0, i}^{1}(\tilde{\mathcal{S N}})$. Moreover, because the group $E_{0, i}^{2}$ is a quotient of $E_{0, i}^{1}$, these maps have the same image in $\mathcal{R P}_{i}^{1}(k) \otimes_{\mathbb{Z}} \mathbb{Z}\left[1 / 2, k[C]^{\times} /\left(k[C]^{\times}\right)^{2}\right]$. The elements in $E_{0, i}^{1}(\tilde{\mathcal{S N}})$ mapping to 0 in $E_{0, i}^{2}(\tilde{\mathcal{S N}})$ come from $E_{1, i}^{1}(\tilde{\mathcal{S N}}) \cong E_{1, i}^{1}\left(\mathrm{SL}_{2}(k[C])\right)$. The elements in $\operatorname{ker}\left(E_{0, i}^{1}(\tilde{\mathcal{S N}}) \rightarrow E_{0, i}^{2}(\tilde{\mathcal{S N}})\right)$ can then be identified exactly with the elements of $\operatorname{coker}\left(E_{1, i}^{2}(\tilde{\mathcal{S N}}) \rightarrow E_{1, i}^{2}\left(\mathrm{SL}_{2}(k[C])\right)\right)$, whence we get an exact sequence

$$
0 \rightarrow \operatorname{coker}\left(E_{1, i}^{2}(\tilde{\mathcal{S \mathcal { N }}}) \rightarrow E_{1, i}^{2}\left(\mathrm{SL}_{2}(k[C])\right)\right) \rightarrow E_{0, i}^{1}(\tilde{\mathcal{S \mathcal { N }}}) \rightarrow E_{0, i}^{2}(\tilde{\mathcal{S \mathcal { N }}}) \rightarrow 0
$$

There is a similar exact sequence

$$
\begin{aligned}
0 & \rightarrow \operatorname{coker}\left(E_{1, i}^{2}(\tilde{\mathcal{S N}}) \rightarrow E_{1, i}^{2}\left(\mathrm{SL}_{2}(k[C])\right)\right) \\
& \rightarrow \operatorname{ker}\left(E_{0, i}^{1}(\tilde{\mathcal{S N}}) \rightarrow E_{0, i}^{1}\left(\mathrm{SL}_{2}(k[C])\right)\right) \\
& \rightarrow \operatorname{ker}\left(E_{0, i}^{2}(\tilde{\mathcal{S N}}) \rightarrow E_{0, i}^{2}\left(\mathrm{SL}_{2}(k[C])\right)\right) \rightarrow 0 .
\end{aligned}
$$

The exact sequence of (4) then gives rise to an exact sequence

$$
\begin{aligned}
\cdots & \rightarrow \mathcal{R P}_{i+1}^{1}(k) \otimes_{\mathbb{Z}} \mathbb{Z}\left[1 / 2, k[C]^{\times} /\left(k[C]^{\times}\right)^{2}\right] \\
& \rightarrow \operatorname{ker}\left(E_{0, i}^{1}(\tilde{\mathcal{S N}}) \rightarrow E_{0, i}^{1}\left(\mathrm{SL}_{2}(k[C])\right)\right) \\
& \rightarrow \operatorname{coker}\left(E_{0, i}^{2}(\tilde{\mathcal{S}}) \rightarrow E_{0, i}^{2}\left(\mathrm{SL}_{2}(k[C])\right)\right) \\
& \rightarrow \mathcal{R P}_{i}^{1}(k) \otimes_{\mathbb{Z}} \mathbb{Z}\left[1 / 2, k[C]^{\times} /\left(k[C]^{\times}\right)^{2}\right] \rightarrow \cdots
\end{aligned}
$$

and the second term is also completely decomposed into stuff coming from the comparison of $E^{2}$-terms, using the exact sequence just above.

Finally, we investigate the map $E_{1, i}^{2}(\tilde{\mathcal{S N}}) \rightarrow E_{1, i}^{2}\left(\mathrm{SL}_{2}(k[C])\right)$. Let $\sigma \in E_{1, i}^{2}(\tilde{\mathcal{S N}})$ be such that it becomes trivial in $E_{1, i}^{2}\left(\mathrm{SL}_{2}(k[C])\right)$. There is a representative $\tilde{\sigma} \in$ $E_{1, i}^{1}(\tilde{\mathcal{S N}})$ with $d^{1} \tilde{\sigma}=0$. Since the maps $E_{p, i}^{1}(\tilde{\mathcal{S N}}) \rightarrow E_{p, i}^{1}\left(\operatorname{SL}_{2}(k[C])\right)$ are isomorphisms for any $p \geq 1$ and any $i$, if the image of $\sigma$ in $E_{1, i}^{2}\left(\operatorname{SL}_{2}(k[C])\right)$ is trivial, then already $\sigma=0$. We see that the map $E_{1, i}^{2}(\tilde{\mathcal{S N}}) \rightarrow E_{1, i}^{2}\left(\mathrm{SL}_{2}(k[C])\right)$ is injective. 
Recall again that all the comparison morphisms $E_{p, i}^{1}(\tilde{\mathcal{S N}}) \rightarrow E_{p, i}^{1}\left(\mathrm{SL}_{2}(k[C])\right)$ are isomorphisms for all $i$ and $p \geq 1$. In particular, the comparison maps on the $E^{2}$ terms discussed above (dealing with kernels and cokernels of the maps induced on $E_{0, i}^{2}$ and $\left.E_{1, i}^{2}\right)$ are the only ones which have a possibly non-trivial kernel or cokernel. In particular, the long exact sequence above gives a complete comparison statement for the $E^{2}$-terms of the two spectral sequences.

(6) By Lemma 6.2. the spectral sequence for $S L_{2}(k[C])$-equivariant homology of $\mathfrak{P}(\mathcal{L})$ also has to degenerate at the $E^{2}$-term, because all the differentials (except the $d_{1, i}^{1}: E_{1, i}^{1} \rightarrow E_{0, i}^{1}$ discussed above) come from the spectral sequence for $\tilde{\mathcal{S N}}$. In particular, $E_{p, q}^{\infty}\left(\mathrm{SL}_{2}(k[C])\right)=E_{p, q}^{2}\left(\mathrm{SL}_{2}(k[C])\right)$ and $E_{p, q}^{\infty}\left(\mathrm{SL}_{2}(k[C])\right) \cong E_{p, q}^{\infty}(\tilde{\mathcal{S}} \tilde{\mathcal{N}})$ for $p \geq 2$.

(7) We now want to promote the exact sequence on $E^{2}$-terms to an exact sequence of the homology groups proper. Obviously, the equivariant map of the model complex into the parabolic component induces a morphism of homology groups $\mathrm{H}_{\bullet}(\tilde{\mathcal{S N}}, \mathbb{Z}[1 / 2]) \rightarrow \mathrm{H}_{\bullet}^{\mathrm{SL}_{2}(k[C])}\left(\mathfrak{P}_{C}(\mathcal{L}), \mathbb{Z}[1 / 2]\right)$ which is compatible with the filtrations and the spectral sequences. The degeneration of the spectral sequence in (6) together with the computations of kernels and cokernels of comparison maps in step (5) imply that

$$
\begin{gathered}
\operatorname{ker}\left(\mathrm{H}_{i}(\tilde{\mathcal{S N}}, \mathbb{Z}[1 / 2]) \rightarrow \mathrm{H}_{i}^{\mathrm{SL}_{2}(k[C])}\left(\mathfrak{P}_{C}(\mathcal{L}), \mathbb{Z}[1 / 2]\right)\right) \\
\cong \operatorname{ker}\left(E_{0, i}^{2}(\tilde{\mathcal{S N}}) \rightarrow E_{0, i}^{2}\left(\mathrm{SL}_{2}(k[C])\right)\right) .
\end{gathered}
$$

Using this identification, there is a natural map

$$
\mathcal{R} \mathcal{P}_{i+1}^{1}(k)\left[k[C]^{\times} /\left(k[C]^{\times}\right)^{2}\right] \rightarrow \operatorname{ker}\left(\mathrm{H}_{i}(\tilde{\mathcal{S N}}, \mathbb{Z}[1 / 2]) \rightarrow \mathrm{H}_{i}^{\mathrm{SL}_{2}(k[C])}\left(\mathfrak{P}_{C}(\mathcal{L}), \mathbb{Z}[1 / 2]\right)\right)
$$

coming from the composition

$$
\mathcal{R} \mathcal{P}_{i+1}^{1}(k)\left[k[C]^{\times} /\left(k[C]^{\times}\right)^{2}\right] \rightarrow E_{0, i}^{1}(\tilde{\mathcal{S N}}) \rightarrow E_{0, i}^{2}(\tilde{\mathcal{S N}})
$$

cf. step (5) above.

From the analysis of the complex $\mathfrak{P}_{C}(\mathcal{L})$ we see that its equivariant homology is detected by the maximal torus and the subgroups $\mathrm{SL}_{2}(k)$ sitting at the special vertices. In particular, there is a natural map

$$
\mathrm{H}_{\bullet}\left(\mathrm{SL}_{2}(k), \mathbb{Z}[1 / 2]\right)\left[k[C]^{\times} /\left(k[C]^{\times}\right)^{2}\right] \rightarrow \mathcal{R} \mathcal{P}_{\bullet}^{1}(k)\left[k[C]^{\times} /\left(k[C]^{\times}\right)^{2}\right]
$$

and the analysis of the spectral sequence shows that this map extends to the required natural map

$$
\mathrm{H}_{i}^{\mathrm{SL}_{2}(k[C])}\left(\mathfrak{P}_{C}(\mathcal{L}), \mathbb{Z}[1 / 2]\right) \rightarrow \mathcal{R} \mathcal{P}_{i}^{1}(k)\left[k[C]^{\times} /\left(k[C]^{\times}\right)^{2}\right]
$$

because all the additional relations come from homology of $\mathrm{N}(k)$ and then are satisfied in the target as well.

Using step (5) again, we can describe the cokernel of the comparison map via an extension

$$
\begin{aligned}
& 0 \rightarrow \operatorname{coker}\left(E_{0, i}^{2}(\tilde{\mathcal{S N}}) \rightarrow E_{0, i}^{2}\left(\mathrm{SL}_{2}(k[C])\right)\right) \rightarrow \\
& \rightarrow \operatorname{coker}\left(\mathrm{H}_{i}(\tilde{\mathcal{S N}}, \mathbb{Z}[1 / 2]) \rightarrow \mathrm{H}_{i}^{\mathrm{SL}_{2}(k[C])}\left(\mathfrak{P}_{C}(\mathcal{L}), \mathbb{Z}[1 / 2]\right)\right) \rightarrow \\
& \rightarrow \operatorname{coker}\left(E_{1, i-1}^{2}(\tilde{\mathcal{S N}}) \rightarrow E_{1, i-1}^{2}\left(\mathrm{SL}_{2}(k[C])\right)\right) \rightarrow 0 .
\end{aligned}
$$

Also from step (5), we know that there is a natural map $\mathcal{R} \mathcal{P}_{i}^{1}(k)\left[k[C]^{\times} /\left(k[C]^{\times}\right)^{2}\right] \rightarrow$ $E_{0, i}^{2}(\tilde{\mathcal{S N}})$ whose kernel also sits in an exact sequence with the same outer terms as above. The natural map induces isomorphisms on the graded pieces, by the computations in step (5). This implies the exactness claim. 
6.4. Components of normalizer type, case $\mathrm{PGL}_{2}$. Now we state the corresponding results on the homology of the normalizer type components for the case of the group $(\mathrm{P}) \mathrm{GL}_{2}$.

Proposition 6.4. Let $k$ be an algebraically closed field, let $\bar{C}$ be a smooth projective curve and denote $C=\bar{C} \backslash\left\{P_{1}, \ldots, P_{s}\right\}$. Let $\mathcal{L}$ be a line bundle on $\bar{C}$ which is 2torsion in the Picard group of $C$, and denote by $\mathfrak{P}_{C}(\mathcal{L})$ the corresponding connected component of the parabolic subcomplex, cf. Proposition 5.8.

Denote $\tilde{\nu}: \tilde{\mathcal{N}} \rightarrow \mathrm{PGL}_{2}(k[C])$ the analogue of the group homomorphism discussed above. The $\tilde{\nu}$-equivariant map $\phi_{\mathcal{L}}: \mathcal{Z}^{s} \rightarrow \mathfrak{P}_{C}(\mathcal{L})$ induces a homomorphism

$$
\mathrm{H}_{\bullet}(\tilde{\mathcal{N}}, \mathbb{Z}[1 / 2]) \cong \mathrm{H}_{\bullet}^{\tilde{\mathcal{N}}}\left(\mathcal{Z}^{s}, \mathbb{Z}[1 / 2]\right) \rightarrow \mathrm{H}_{\bullet}^{\mathrm{PGL}_{2}(k[C])}\left(\mathfrak{P}_{C}(\mathcal{L}), \mathbb{Z}[1 / 2]\right)
$$

such that there is a long exact sequence of $\mathbb{Z}[1 / 2]$-modules

$$
\cdots \rightarrow \mathcal{P}_{i+1}^{1}(k) \rightarrow \mathrm{H}_{i}(\tilde{\mathcal{N}}, \mathbb{Z}[1 / 2]) \rightarrow \mathrm{H}_{i}^{\mathrm{PGL}_{2}(k[C])}\left(\mathfrak{P}_{C}(\mathcal{L}), \mathbb{Z}[1 / 2]\right) \rightarrow \mathcal{P}_{i}^{1}(k) \rightarrow \cdots
$$

The arguments are similar to the ones used for $\mathrm{SL}_{2}$ above, and we omit a detailed proof: the proof first proceeds through an analogue of Lemma 6.2 for the group $\tilde{\mathcal{N}}$, and then mainly uses the $\mathrm{PGL}_{2}$-part of Proposition 5.8 in much the same way as happened in the proof of Proposition 6.3

The groups $\mathcal{P}_{i}^{1}(k)$ appearing above are the generalized scissors congruence groups of Dup01, cf. also Section A. Using the long exact sequence connecting homology of the normalizer $\mathrm{N}(k)$, homology of $\mathrm{SL}_{2}(k)$ and the scissors congruence groups $\mathcal{P}_{i}^{1}(k)$, we can simplify the above result further and describe the parabolic homology as the following pushout, all with $\mathbb{Z}[1 / 2]$-coefficients:

$$
\mathrm{H}_{\bullet}^{\mathrm{PGL}_{2}(k[C])}\left(\mathfrak{P}_{C}(\mathcal{L})\right) \cong \mathrm{H}_{\bullet}(\tilde{\mathcal{N}}) \oplus_{\mathrm{H}_{\bullet}(\mathrm{N}(k))} \mathrm{H}_{\bullet}\left(\mathrm{PGL}_{2}(k)\right) .
$$

6.5. Functoriality. We have described the $\mathrm{SL}_{2}(k[C])$-equivariant homology of the parabolic subcomplex $\mathfrak{P}_{C}$ above. We now want to describe the behaviour of parabolic homology under morphisms of curves. In particular we want to compute the colimit of these homologies to $\mathrm{SL}_{2}(k(C))$ and $\mathrm{SL}_{2}(\overline{k(C)})$.

Proposition 6.5. Let $k$ be an algebraically closed field. Let $f: \bar{D} \rightarrow \bar{C}$ be a finite morphism of smooth projective curves over $k$, let $P_{1}, \ldots, P_{s}$ be points on $\bar{C}$, and let $Q_{1}, \ldots, Q_{t}$ be points on $\bar{D}$ not in the preimage of the $P_{i}$. Set $C=\bar{C} \backslash\left\{P_{1}, \ldots, P_{s}\right\}$ and $D=\bar{D} \backslash\left(\left\{f^{-1}\left(\left\{P_{1}, \ldots, P_{s}\right\}\right) \cup\left\{Q_{1}, \ldots, Q_{t}\right\}\right)\right.$. Denote by $\mathfrak{P}_{C}$ and $\mathfrak{P}_{D}$ the parabolic subcomplexes in $\mathfrak{X}_{C}$ and $\mathfrak{X}_{D}$, respectively. Then we have the following assertions for the morphism

$$
f^{*}: \mathrm{H}_{\bullet}^{\mathrm{SL}_{2}(k[C])}\left(\mathfrak{P}_{C}\right) \rightarrow \mathrm{H}_{\bullet}^{\mathrm{SL}_{2}(k[D])}\left(\mathfrak{P}_{D}\right)
$$

induced from the morphism of Proposition 3.12, where we use $\mathbb{Z}[1 / 2]$-coefficients throughout:

(1) The composition

$$
\mathcal{K}(C) \rightarrow \pi_{0}\left(\mathrm{SL}_{2}(k[C]) \backslash \mathfrak{P}_{C}\right) \stackrel{\pi_{0}\left(f^{*}\right)}{\longrightarrow} \pi_{0}\left(\mathrm{SL}_{2}(k[D]) \backslash \mathfrak{P}_{D}\right) \rightarrow \mathcal{K}(D)
$$

is induced from pullback of line bundles $f^{*}: \operatorname{Pic}(C) \rightarrow \operatorname{Pic}(D)$, where the first and last map are the bijections of Lemma 5.2.

(2) For each line bundle $\mathcal{L}$ on $\bar{C}$, there is an induced map

$$
f_{\mathcal{L}}^{*}: \mathrm{H}_{\bullet}^{\mathrm{SL}_{2}(k[C])}\left(\mathfrak{P}_{C}(\mathcal{L})\right) \rightarrow \mathrm{H}_{\bullet}^{\mathrm{SL}_{2}(k[D])}\left(\mathfrak{P}_{D}\left(f^{*}(\mathcal{L})\right)\right) .
$$

The map $f^{*}$ is the direct sum of these.

(3) If $\mathcal{L}$ is not 2 -torsion in $\operatorname{Pic}(D)$, then $f_{\mathcal{L}}^{*}$ is identified via the isomorphisms of Proposition 6.1 with the natural map

$$
\mathrm{H}_{\bullet}\left(k[C]^{\times}\right) \rightarrow \mathrm{H}_{\bullet}\left(k[D]^{\times}\right) .
$$


(4) If $\mathcal{L}$ is not 2-torsion in $\operatorname{Pic}(C)$ but becomes 2 -torsion in $\operatorname{Pic}(D)$, then $f_{\mathcal{L}}^{*}$ is identified with the composition

$$
\mathrm{H}_{\bullet}\left(k[C]^{\times}\right) \rightarrow \mathrm{H}_{\bullet}(\tilde{\mathcal{S N}}) \rightarrow \mathrm{H}_{\bullet}^{\mathrm{SL}_{2}(k[C])}\left(\mathfrak{P}_{D}\left(f^{*} \mathcal{L}\right)\right),
$$

where the first map is the inclusion of diagonal matrices into monomial matrices, and the second map is the morphism from Proposition 6.3.

(5) If $\mathcal{L}$ is 2 -torsion in $\operatorname{Pic}(C)$, then $f_{\mathcal{L}}^{*}$ sits in a commutative ladder connecting the respective long exact sequences of Proposition 6.3 for $\mathfrak{P}_{C}$ and $\mathfrak{P}_{D}$. The other two types of maps of the commutative ladder, $\mathrm{H}_{\bullet}\left(\tilde{\mathcal{S N}}_{C}\right) \rightarrow \mathrm{H}_{\bullet}\left(\tilde{\mathcal{S N}}_{D}\right)$ and

$$
\mathcal{R P}_{\bullet}^{1}(k) \otimes_{\mathbb{Z}} \mathbb{Z}\left[1 / 2, k[C]^{\times} /\left(k[C]^{\times}\right)^{2}\right] \rightarrow \mathcal{R P}^{1} \bullet(k) \otimes_{\mathbb{Z}} \mathbb{Z}\left[1 / 2, k[D]^{\times} /\left(k[D]^{\times}\right)^{2}\right]
$$

are induced by the natural map $k[C]^{\times} \rightarrow k[D]^{\times}$.

Proof. The result is a direct consequence of Proposition 5.9 and the computations in Proposition 6.1 and Proposition 6.3.

Obviously, there is a similar functoriality result for the case $\mathrm{PGL}_{2}$ where again all relevant maps are induced from the natural one $f^{*}: k[C]^{\times} \rightarrow k[D]^{\times}$.

Definition 6.6. Let $k$ be an algebraically closed field, let $\bar{C}$ be a smooth projective curve over $k$, and set $C=\bar{C} \backslash\left\{P_{1}, \ldots, P_{s}\right\}$. We define the parabolic homology of $\mathrm{SL}_{2}(k(C))$ to be

$$
\widehat{\mathrm{H}}_{\bullet}\left(\mathrm{SL}_{2}(k(C)), \mathbb{Z} / \ell\right)=\underset{S \subseteq \bar{C}(k)}{\operatorname{colim}} \mathrm{H}_{\bullet}^{\mathrm{SL}_{2}(k[\bar{C} \backslash S])}(\mathfrak{P} \bar{C} \backslash S, \mathbb{Z} / \ell),
$$

where the colimit is taken over all finite sets $S$ of closed points of $\bar{C}$, ordered by inclusion.

As a direct consequence of Proposition 6.5, we can describe the parabolic homology for function fields of curves.

Proposition 6.7. Let $k$ be an algebraically closed field, let $C$ be a smooth curve over $k$ and let $\ell$ be an odd prime different from the characteristic of $k$. Then we have the following exact sequence

$$
\begin{aligned}
& \cdots \rightarrow \mathcal{R} \mathcal{P}_{i+1}^{1}(k, \mathbb{Z} / \ell)\left[k(C)^{\times} /\left(k(C)^{\times}\right)^{2}\right] \rightarrow \mathrm{H}_{i}(\mathrm{~N}(k(C)), \mathbb{Z} / \ell) \rightarrow \\
& \rightarrow \widehat{\mathrm{H}}_{i}\left(\mathrm{SL}_{2}(k(C)), \mathbb{Z} / \ell\right) \rightarrow \mathcal{R} \mathcal{P}_{i}^{1}(k, \mathbb{Z} / \ell)\left[k(C)^{\times} /\left(k(C)^{\times}\right)^{2}\right] \rightarrow \cdots,
\end{aligned}
$$

where $\mathrm{N}(k(C))$ denotes the normalizer of a maximal torus in $\mathrm{SL}_{2}(k(C))$.

6.6. Low-dimensional example calculations. We discuss low-dimensional special cases of the above computations in Proposition 6.1 and Proposition 6.3. In low degrees, we can say more about the precise relation between the homology of $\mathrm{SL}_{2}(k)$ and the homology of the normalizer of the maximal torus $\mathrm{N}(k)$.

We start with the degree 1 case:

Corollary 6.8. Let $k$ be an algebraically closed field, let $\bar{C}$ be a smooth projective curve and denote $C=\bar{C} \backslash\left\{P_{1}, \ldots, P_{s}\right\}$. There is an isomorphism

$$
\mathrm{H}_{1}^{\mathrm{SL}_{2}(k[C])}\left(\mathfrak{P}_{C}, \mathbb{Z}[1 / 2]\right) \cong \bigoplus_{[\mathcal{L}] \in \mathcal{K}(C), 2 \mathcal{L} \neq 0} k[C]^{\times} \otimes_{\mathbb{Z}} \mathbb{Z}[1 / 2] .
$$

In the limit, we have $\widehat{\mathrm{H}}_{1}\left(\mathrm{SL}_{2}(k(C)), \mathbb{Z}[1 / 2]\right)=0$.

Proof. By Proposition 6.1, if $\mathcal{L}$ is a line bundle on $\bar{C}$ which is not 2-torsion in $\operatorname{Pic}(C)$, then

$$
\mathrm{H}_{1}^{\mathrm{SL}_{2}(k[C])}\left(\mathfrak{P}_{C}(\mathcal{L}), \mathbb{Z}[1 / 2]\right) \cong k[C]^{\times} \otimes_{\mathbb{Z}} \mathbb{Z}[1 / 2] .
$$


Now let $\mathcal{L}$ be a line bundle on $\bar{C}$ which is 2 -torsion in $\operatorname{Pic}(C)$. We investigate the components of the exact sequence computing $\mathrm{H}_{1}^{\mathrm{SL}_{2}(k[C])}\left(\mathfrak{P}_{C}(\mathcal{L}), \mathbb{Z}[1 / 2]\right)$. The group $\tilde{\mathcal{S N}}$ is an extension of the free abelian group $\tilde{\mathcal{S T}}$ by a 2 -torsion group $G$. Obviously, $\mathrm{H}_{1}\left(G, \mathrm{H}_{0}(\tilde{\mathcal{S T}}, \mathbb{Z}[1 / 2])\right)=0$. But

$$
\mathrm{H}_{0}\left(G, \mathrm{H}_{1}(\tilde{\mathcal{S T}}, \mathbb{Z}[1 / 2])\right) \cong \mathrm{H}_{0}\left(G, \tilde{\mathcal{S T}} \otimes_{\mathbb{Z}} \mathbb{Z}[1 / 2]\right)=0
$$

because any element of $G$ acts by inversion followed by multiplication with some element, whence the coinvariants are 2-torsion. The Hochschild-Serre spectral sequence for the extension then implies $\mathrm{H}_{1}(\tilde{\mathcal{S}} \tilde{\mathcal{N}}, \mathbb{Z}[1 / 2])=0$. Similarly, the computations in Hut11b] imply that $\mathcal{R P}_{1}^{1}(k)=0$, cf. Section $\mathrm{A}$, and therefore

$$
\mathcal{R} \mathcal{P}_{1}^{1}(k) \otimes_{\mathbb{Z}} \mathbb{Z}\left[1 / 2, k(C)^{\times} /\left(k(C)^{\times}\right)^{2}\right]=0 .
$$

The exact sequence of Proposition 6.3 then implies $\mathrm{H}_{1}^{\mathrm{SL}_{2}(k[C])}\left(\mathfrak{P}_{C}(\mathcal{L}), \mathbb{Z}[1 / 2]\right)=0$.

The claim then follows from Lemma 5.2. The limit claim follows from the above and Proposition 6.7

Corollary 6.9. Let $k$ be an algebraically closed field, let $\bar{C}$ be a smooth projective curve and denote $C=\bar{C} \backslash\left\{P_{1}, \ldots, P_{s}\right\}$. There is an exact sequence

$$
\begin{aligned}
& 0 \rightarrow \mathrm{H}_{3}(\tilde{\mathcal{S N}}, \mathbb{Z}[1 / 2]) \rightarrow \mathrm{H}_{3}^{\mathrm{SL}_{2}(k[C])}\left(\mathfrak{P}_{C}(\mathcal{L}), \mathbb{Z}[1 / 2]\right) \rightarrow \\
& \quad \rightarrow \mathcal{R} \mathcal{P}_{3}^{1}(k) \otimes_{\mathbb{Z}} \mathbb{Z}\left[1 / 2, k[C]^{\times} /\left(k[C]^{\times}\right)^{2}\right] \rightarrow \\
& \rightarrow \mathrm{H}_{2}(\tilde{\mathcal{S N}}, \mathbb{Z}[1 / 2]) \rightarrow \mathrm{H}_{2}^{\mathrm{SL}_{2}(k[C])}\left(\mathfrak{P}_{C}(\mathcal{L}), \mathbb{Z}[1 / 2]\right) \rightarrow 0 .
\end{aligned}
$$

The maps are the ones appearing in the Bloch-Wigner exact sequence, cf. Hut11b.

\section{Appendix A. Refined scissors CONGRUence groups}

The following section provides a recollection on the exact sequence relating homology of $\mathrm{SL}_{2}(k)$, homology of the normalizer of a maximal torus $\mathrm{N}(k)$ and suitable generalizations of pre-Bloch groups $\mathcal{R P}^{1} \bullet(k)$. The material is well-known from the work of Bloch, Suslin, Dupont, Sah, Hutchinson and others, cf. Sus90, Dup01, Hut11b. As the statements are somehow scattered over the literature and not usually stated in the generality needed, we provide a detailed review of the definitions of the refined scissors congruence groups (or point configuration groups) $\mathcal{R P}^{1} \cdot(k)$ and the proof of the relevant exact sequences for homology of $\mathrm{SL}_{2}(k)$.

A.1. Points on the projective line. Let $F$ be an infinite field. Consider the standard action of the general linear group $\mathrm{GL}_{2}(F)$ on the $F$-points of the projective line $\mathbb{P}^{1}(F)$ given by

$$
\left(\begin{array}{cc}
a & b \\
c & d
\end{array}\right) \cdot z \mapsto \frac{a z+b}{c z+d}
$$

As the center obviously acts trivially, the group action factors through $\operatorname{PGL}_{2}(F)$. Restriction to determinant 1 provides the standard group actions of $\mathrm{SL}_{2}(F)$ and $\mathrm{PSL}_{2}(F)$ on $\mathbb{P}^{1}(F)$.

Definition A.1. Let $F$ be an infinite field.

(1) Denote by $C_{\bullet}(F)$ the complex of points on $\mathbb{P}^{1}$, which in degree $n$ has the free abelian group $C_{n}(F)$ generated by $(n+1)$-tuples $\left(x_{0}, \ldots, x_{n}\right)$ of distinct points on $\mathbb{P}^{1}(F)$.

(2) Denote by $C_{\bullet}^{\text {alt }}(F)$ the alternating complex of points on $\mathbb{P}^{1}$, where $C_{n}^{\text {alt }}(F)$ is the free abelian group generated by $(n+1)$-tuples $\left(x_{0}, \ldots, x_{n}\right)$ of points on $\mathbb{P}^{1}(F)$ modulo the identifications

$$
\left(x_{\pi(0)}, \ldots, x_{\pi(n)}\right)=\operatorname{sgn} \pi \cdot\left(x_{0}, \ldots, x_{n}\right),
$$

where $\pi$ is any permutation of the set of indices $\{0, \ldots, n\}$. 
In both the above cases, the differential is given by the obvious

$$
d\left(x_{0}, \ldots, x_{n}\right)=\sum_{i=0}^{n}(-1)^{i}\left(x_{0}, \ldots, \widehat{x}_{i}, \ldots, x_{n}\right) .
$$

The complexes are augmented by mapping

$$
C_{0}(F)=C_{0}^{\text {alt }}(F) \rightarrow \mathbb{Z}:\left(x_{0}\right) \mapsto 1
$$

We recall the well-known acyclicity lemma, cf. [Knu01, Lemma 2.3.2], Dup01, Lemma 3.6] or any of a huge number of further possible sources.

Lemma A.2. The augmented complexes $C_{\bullet}^{(\text {alt })}(F) \rightarrow \mathbb{Z} \rightarrow 0$ of points on $\mathbb{P}^{1}$ are acyclic.

Proof. Let $\sum_{j=1}^{N} n_{j}\left(x_{j, 0}, \ldots, x_{j, q}\right)$ be a $q$-cycle in $C_{\bullet}(F)$. Since $F$ is infinite, there exists a point $x \in \mathbb{P}^{1}(F)$ different from any of the points $x_{j, q}$. Then

$$
d\left(\sum_{j=1}^{N} n_{j}\left(x, x_{j, 0}, \ldots, x_{j, q}\right)\right)=\sum_{j=1}^{N} n_{j}\left(x_{j, 0}, \ldots, x_{j, q}\right) .
$$

For the alternating complexes, one can even choose a global base-point $x \in \mathbb{P}^{1}$ and use the global contraction

$$
s_{q}: C_{q}^{\text {alt }}(F) \rightarrow C_{q+1}^{\text {alt }}(F):\left(x_{0}, \ldots, x_{q}\right) \mapsto\left(x, x_{0}, \ldots, x_{q}\right), s_{-1}(1)=(x) .
$$

Contractibility implies that the $\mathrm{SL}_{2}(F)$-equivariant homology of the complexes of points on $\mathbb{P}^{1}$ can indeed be identified with group homology of $\mathrm{SL}_{2}(F)$.

Corollary A.3. Let $F$ be an infinite field, and let $\Gamma$ be any one of the groups $(\mathrm{P}) \mathrm{GL}_{2}(F)$ or $(\mathrm{P}) \mathrm{SL}_{2}(F)$. Then the augmentation induces isomorphisms

$$
\mathrm{H}_{\bullet}\left(\Gamma, C_{\bullet}^{(\text {alt })}(F)\right) \stackrel{\cong}{\longrightarrow} \mathrm{H}_{\bullet}(\Gamma, \mathbb{Z}) .
$$

The hyperhomology spectral sequence associated to $\mathrm{H}_{\bullet}\left(\Gamma, C_{\bullet}^{(\text {alt })}\right)$ provides the relation between homology of $\Gamma$, the normalizer of a maximal torus in $\Gamma$ and the refined scissors congruence groups.

There is a notion of decomposability of point configurations in projective $n$-space, cf. Dup01, p. 126]. Using $\mathbb{Z}[1 / 2]$-coefficients and noticing that $(x, x)=-(x, x)$ implies that $(x, x)$ is 2-torsion, we can simplify the notion of decomposability for our purposes: for the projective line, an $(n+1)$-tuple of points $\left(x_{0}, \ldots, x_{n}\right)$ is decomposable if and only if its support contains at most two points. In particular, a non-trivial element in $C_{q}^{(\text {alt })}(F)$ is decomposable if and only if $q \leq 1$. The decomposable subcomplex of the complex of points is defined as follows:

$$
F_{0} C_{q}^{(\text {alt })}(F)= \begin{cases}C_{q}^{(\text {alt })}(F) & q \leq 1 \\ 0 & \text { otherwise }\end{cases}
$$

It is obviously stable under the action of $(\mathrm{P}) \mathrm{GL}_{2}(F)$.

Definition A.4. Let $F$ be an infinite field. The refined scissors congruence groups (or refined point configuration groups) are defined as

$$
\mathcal{R P}_{\bullet}^{1}(F):=\mathrm{H}_{\bullet}\left(\mathrm{SL}_{2}(F), C_{\bullet}^{\text {alt }}(F) / F_{0} C_{\bullet}^{\text {alt }}(F)\right)
$$

The scissors congruence groups (or point configuration groups) are defined as

$$
\mathcal{P}_{\bullet}^{1}(F):=\mathrm{H}_{\bullet}\left(\mathrm{PGL}_{2}(F), C_{\bullet}^{\text {alt }}(F) / F_{0} C_{\bullet}^{\text {alt }}(F)\right)
$$


Remark A.5. For considerations with finite coefficients, we can define similar groups

$$
\mathcal{R P}_{\bullet}^{1}(F, \mathbb{Z} / \ell):=\mathrm{H}_{\bullet}\left(\mathrm{SL}_{2}(F), C_{\bullet}^{\text {alt }}(F) / F_{0} C_{\bullet}^{\text {alt }}(F) \otimes_{\mathbb{Z}} \mathbb{Z} / \ell\right)
$$

Remark A.6. The notation $\mathcal{P}_{q}^{1}(F)$ is taken from Dup01. The notation $\mathcal{R P}_{q}^{1}(F)$ for the refined scissors congruence groups is a combination of the notation from Dup01 and Hut11b. In Hut11b], only the groups $\mathcal{R P}_{3}^{1}(F)$ are considered (and simply denoted $\left.\mathcal{R} \mathcal{P}_{1}(F)\right)$.

Proposition A.7. If $F$ is a quadratically closed field, the natural map $\mathrm{SL}_{2}(F) \rightarrow$ $\mathrm{PGL}_{2}(F)$ induces isomorphisms

$$
\mathcal{R P}_{\bullet}^{1}(F) \otimes_{\mathbb{Z}} \mathbb{Z}[1 / 2] \stackrel{\cong}{\longrightarrow} \mathcal{P}_{\bullet}^{1}(F) \otimes_{\mathbb{Z}} \mathbb{Z}[1 / 2] .
$$

Proof. The natural map factors as $\mathrm{SL}_{2}(F) \rightarrow \mathrm{PSL}_{2}(F) \rightarrow \mathrm{PGL}_{2}(F)$, and we will show that any of these homomorphisms induces an isomorphism on equivariant homology with coefficients in $C_{\bullet}^{\text {alt }}(F)$.

If $F$ is quadratically closed, then $\operatorname{PSL}_{2}(F)$ and $\mathrm{PGL}_{2}(F)$ are in fact the same groups, because any matrix $M \in \mathrm{PGL}_{2}(F)$ can be scaled, using the diagonal matrix $\operatorname{diag}\left(\operatorname{det} M^{\frac{1}{2}}, \operatorname{det} M^{\frac{1}{2}}\right)$, to a matrix in $\operatorname{PSL}_{2}(F)$. Therefore, the second homomorphism obviously induces an isomorphism on homology.

The homomorphism $\mathrm{SL}_{2}(F) \rightarrow \mathrm{PSL}_{2}(F)$ is surjective and has kernel $\{ \pm I\} \cong$ $\mathbb{Z} / 2 \mathbb{Z}$. Since the kernel acts in fact trivially on the complexes $C_{\bullet}^{\text {alt }}(F)$, the projection hence induces an isomorphism on homology with $\mathbb{Z}[1 / 2]$-coefficients.

A.2. The hyperhomology spectral sequence. The hyperhomology spectral sequence for the action of $\mathrm{SL}_{2}(F)$ on the complexes $C_{\bullet}^{(\text {alt })}(F)$ provides some insights into the structure of the homology of $\mathrm{SL}_{2}(F)$, which we discuss next.

We first recall a suitable resolution of the complex $F_{0} C_{\bullet}^{\text {alt }}(F)$ from Dup01, Proposition 13.22]. The complex $D_{\bullet}(F)$ is the following complex

$$
D \bullet(F)=\bigoplus_{x \in \mathbb{P}^{1}(F)}\left(\mathbb{Z}\left[\mathbb{P}^{1}(F) \backslash\{x\}\right] \rightarrow \mathbb{Z}\right),
$$

with the first term sitting in degree 1 and the differential given by the minus the augmentation $y \mapsto-1$. This is a shifted version of the augmented complex, hence the additional sign. The complex $E_{\bullet}(F)$ is defined to be

$$
E_{\bullet}(F):=\bigoplus_{x, y \in \mathbb{P}^{1}(F), x \neq y} \mathbb{Z}
$$

concentrated in degree 1 . Note that the index set is the set of unordered pairs of distinct elements in $\mathbb{P}^{1}(F)$.

Lemma A.8. There is a map of complexes $F_{0} C_{\bullet}^{\text {alt }}(F) \rightarrow D_{\bullet}(F)$ given in degree 0 by $(x) \mapsto 1_{x}$ and in degree 1 by $(x, y) \mapsto(y)_{x}-(x)_{y}$.

There is a map of complexes $D_{\bullet}(F) \rightarrow E_{\bullet}(F)$ given in degree 0 by the 0 -map and in degree 1 by $(x)_{y} \mapsto 1_{(x, y)}$.

With these maps, there is an exact sequence of complexes

$$
0 \rightarrow F_{0} C_{\bullet}^{\text {alt }}(F) \otimes_{\mathbb{Z}} \mathbb{Z}[1 / 2] \rightarrow D_{\bullet}(F) \otimes_{\mathbb{Z}} \mathbb{Z}[1 / 2] \rightarrow E_{\bullet}(F) \otimes_{\mathbb{Z}} \mathbb{Z}[1 / 2] \rightarrow 0 .
$$

Proof. The first claim is simply that $(x, y) \mapsto(y)_{x}-(x)_{y} \mapsto-1_{x}+1_{y}$ and $(x, y) \mapsto$ $(y)-(x) \mapsto 1_{y}-1_{x}$ are the same map.

The second claim is trivially true since $E_{\bullet}(F)$ is concentrated in degree 1 .

We discuss injectivity of the first map: in degree 0 , the map is $(x) \mapsto 1_{x}$ which is obviously injective. In degree 1 , the map is $(x, y) \mapsto(y)_{x}-(x)_{y}$. If $x=y$, then the image is zero, but the element $(x, x)=-(x, x)$ is 2 -torsion, hence also 0 . Injectivity in degree 1 follows from this. 
Surjectivity of the second map is clear, since $1_{(x, y)}$ is in the image of $(x)_{y}$.

It remains to see exactness in the middle. In degree 0 , exactness in the middle is clear: the last term is 0 and the first map $(x) \mapsto 1_{x}$ is an isomorphism. In degree 1 , the composition is obviously 0 , since $(x, y) \mapsto 1_{(x, y)}-1_{(y, x)}$. For an element $c$ in $D_{\bullet}(F)$, the $(x, y)$-component of its image in $E_{\bullet}(F)$ is trivial if the elements $(x)_{y}$ and $(y)_{x}$ occur with opposite multiplicities. This implies exactness in the middle.

We now can identify the homology of the complex $F_{0} C_{\bullet}^{\text {alt }}(F)$ with the homology of the normalizer, as in [Dup01, Proposition 13.22].

Lemma A.9. Let $F$ be an infinite field.

(1) The complex $D_{\bullet}(F)$ is acyclic.

(2) There is an induced isomorphism

$$
\mathrm{H}_{\bullet}\left(\mathrm{SL}_{2}(F), F_{0} C^{\text {alt }}(F) \otimes_{\mathbb{Z}} \mathbb{Z}[1 / 2]\right) \cong \mathrm{H}_{\bullet}(\mathrm{N}(F), \mathbb{Z}[1 / 2]),
$$

where $\mathrm{N}(F)$ is the normalizer of a maximal torus in $\mathrm{SL}_{2}(F)$.

Proof. (1) The degree 1 part of $D_{\bullet}(F)$ is the $\mathrm{SL}_{2}(F)$-module

$$
\bigoplus_{x \in \mathbb{P}^{1}(F)} \mathbb{Z}\left[\mathbb{P}^{1}(F) \backslash\{x\}\right] \cong \operatorname{Ind}_{\mathrm{B}_{2}(F)}^{\mathrm{SL}_{2}(F)} \mathbb{Z}\left[\mathbb{P}^{1}(F) \backslash\{\infty\}\right] \cong \operatorname{Ind}_{\mathrm{T}(F)}^{\mathrm{SL}_{2}(F)} \mathbb{Z} .
$$

In particular, there is an induced isomorphism

$$
\mathrm{H}_{\bullet}\left(\mathrm{SL}_{2}(F), D_{1}(F)\right) \cong \mathrm{H}_{\bullet}(\mathrm{T}(F), \mathbb{Z}) .
$$

Similarly, we can identify the degree 0 part of the complex as

$$
\bigoplus_{x \in \mathbb{P}^{1}(F)} \mathbb{Z} \cong \operatorname{Ind}_{\mathrm{B}_{2}(F)}^{\mathrm{SL}_{2}(F)} \mathbb{Z}
$$

which yields an induced isomorphism

$$
\mathrm{H}_{\bullet}\left(\mathrm{SL}_{2}(F), D_{0}(F)\right) \cong \mathrm{H}_{\bullet}\left(\mathrm{B}_{2}(F), \mathbb{Z}\right) .
$$

Since $F$ is infinite, we can identify the homology of the Borel subgroup $\mathrm{B}_{2}(F)$ with the homology of the maximal torus, i.e., the natural projection $\mathrm{B}_{2}(F) \rightarrow$ $\mathrm{T}(F)$ induces an isomorphism $\mathrm{H}_{\bullet}\left(\mathrm{B}_{2}(F), \mathbb{Z}\right) \cong \mathrm{H}_{\bullet}(\mathrm{T}(F), \mathbb{Z})$. The differential $d_{1}$ : $\mathrm{H}_{\bullet}(\mathrm{T}, \mathbb{Z}) \rightarrow \mathrm{H}_{\bullet}(\mathrm{T}, \mathbb{Z})$ is the identity, because the differential of the complex $d_{1}$ is induced from the natural inclusion $\mathrm{T}(F) \rightarrow \mathrm{B}_{2}(F)$ on the stabilizer groups. Therefore, the complex $D_{\bullet}(F)$ is acyclic.

(2) The result will be proved using the long exact sequence of homology groups associated to the exact sequence of complexes from Lemma A.8. From (1), it suffices to show that the homology of $E_{\bullet}(F)$ is identified with a shift of the homology of the normalizer.

The action of $\mathrm{SL}_{2}(F)$ on $E_{1}(F)=\bigoplus_{x \neq y} \mathbb{Z}$ is transitive, we can choose $\{0, \infty\}$ as orbit representative. The stabilizer of the unordered pair $\{0, \infty\}$ is the normalizer $\mathrm{N}(k)$ : in addition to the diagonal matrices stabilizing both 0 and $\infty$, the Weyl group generator

$$
w=\left(\begin{array}{cc}
0 & 1 \\
-1 & 0
\end{array}\right)
$$

stabilizes $\{0, \infty\}$ setwise. Therefore, there are induced isomorphisms

$$
\mathrm{H}_{i+1}\left(\mathrm{SL}_{2}(F), E_{\bullet}(F)\right) \cong \mathrm{H}_{i}(\mathrm{~N}(F), \mathbb{Z}) \text {. }
$$

From the complex $C_{\bullet}^{\text {alt }}(F)$, we now obtain the required long exact sequence connecting homology of $\mathrm{SL}_{2}(F)$ to the homology of $\mathrm{N}(F)$ and the refined scissors congruence groups $\mathcal{R P}_{\bullet}^{1}(F)$. 
Proposition A.10. Let $F$ be an infinite field.

(1) There is a long exact sequence

$$
\cdots \rightarrow \mathrm{H}_{\bullet}(\mathrm{N}(F), \mathbb{Z}[1 / 2]) \rightarrow \mathrm{H}_{\bullet}\left(\mathrm{SL}_{2}(F), \mathbb{Z}[1 / 2]\right) \rightarrow \mathcal{R} \mathcal{P}_{\bullet}^{1}(F) \rightarrow \cdots
$$

where $\mathrm{N}(F)$ denotes the normalizer of a maximal torus in $\mathrm{SL}_{2}(F)$.

(2) There is a long exact sequence

$$
\cdots \rightarrow \mathrm{H}_{\bullet}\left(\mathrm{N}^{\prime}(F), \mathbb{Z}[1 / 2]\right) \rightarrow \mathrm{H}_{\bullet}\left(\mathrm{PGL}_{2}(F), \mathbb{Z}[1 / 2]\right) \rightarrow \mathcal{P}_{\bullet}^{1}(F) \rightarrow \cdots
$$

where $\mathrm{N}^{\prime}(F)$ denotes the normalizer of a maximal torus in $\mathrm{PGL}_{2}(F)$.

(3) There are similar long exact sequences with $\mathbb{Z} / \ell$-coefficients, $\ell$ an odd prime.

Proof. Consider the exact sequence of complexes

$$
0 \rightarrow F_{0} C_{\bullet}^{\text {alt }}(F) \rightarrow C_{\bullet}^{\text {alt }}(F) \rightarrow C_{\bullet}^{\text {alt }}(F) / F_{0} C_{\bullet}^{\text {alt }}(F) \rightarrow 0 .
$$

The equivariant homology of $F_{0} C_{\bullet}^{\text {alt }}(F)$ is identified with the homology of the normalizer by Lemma A.9. The equivariant homology of $C_{\bullet}^{\text {alt }}(F)$ is identified with the homology of the respective group $\mathrm{SL}_{2}(F)$ or $\mathrm{PGL}_{2}(F)$ by Corollary A.3. The identification of equivariant homology of the quotient $C_{\bullet}^{\text {alt }}(F) / F_{0} C_{\bullet}^{\text {alt }}(F)$ with refined scissors congruence groups is built into Definition A.4.

Remark A.11. Note that the groups $\mathcal{R P}_{\bullet}^{1}(F ; \mathbb{Z} / \ell)$ with finite coefficients are not simply obtained by tensoring $\mathcal{R P}^{1}(F) \otimes_{\mathbb{Z}} \mathbb{Z} / \ell$, but instead by tensoring the defining complex $C_{\bullet}^{\text {alt }}(F)$ with $\mathbb{Z} / \ell$ and then computing equivariant homology.

We make some remarks on the relation between the spectral sequences associated to $C_{\bullet}(F)$ and $C_{\bullet}^{\text {alt }}(F)$. Note first that there is a natural map $C_{\bullet}(F) \rightarrow C_{\bullet}^{\text {alt }}(F)$ given by the identity on tuples of pairwise distinct points. Both complexes are acyclic, hence their $\mathrm{SL}_{2}(F)$-equivariant homology can be identified with group homology of $\mathrm{SL}_{2}(F)$ with constant coefficients. The map between the two complexes then provides a map between spectral spectral sequences converging to $\mathrm{H}_{\bullet}\left(\mathrm{SL}_{2}(F), \mathbb{Z}[1 / 2]\right)$. The spectral sequence associated to $C_{\bullet}(F)$ is the one usually discussed, see for example [Knu01, Theorem 3.2.2], Dup01, Theorem 8.19] or [Hut11b. The spectral sequence for $C_{\bullet}^{\text {alt }}(F)$ as discussed above seems to appear only in Dup01, Chapter $15]$.

Indexing the $E^{1}$-term as $E_{p, q}^{1}=\mathrm{H}_{p}\left(\mathrm{SL}_{2}(F), C_{q}\right)$ and working as always with $\mathbb{Z}[1 / 2]$-coefficients, the $E^{1}$-terms of both spectral sequences are both concentrated in the $p=0$ column as well as the two lines $q=0$ and $q=1$. From the computations in [Hut11b, Section 4], we see that for the complex $C \bullet(F)$, we have

$$
E_{p, 0}^{2} \cong \mathrm{H}_{\bullet}(\mathrm{N}(F), \mathbb{Z}[1 / 2]),
$$

using that with $\mathbb{Z}[1 / 2]$-coefficients, the Hochschild-Serre spectral sequence associated to the extension $1 \rightarrow \mathrm{T}(F) \rightarrow \mathrm{N}(F) \rightarrow \mathbb{Z} / 2 \rightarrow 0$ degenerates to an isomorphism

$$
\mathrm{H}_{\bullet}(\mathrm{N}(F), \mathbb{Z}[1 / 2]) \cong \mathrm{H}_{0}\left(\mathbb{Z} / 2, \mathrm{H}_{\bullet}(\mathrm{T}(F), \mathbb{Z}[1 / 2])\right) .
$$

We also saw above that for the complex $C^{\text {alt }}(F)$, we have

$$
E_{p, 0}^{2, \text { alt }} \cong \mathrm{H}_{\bullet}(\mathrm{N}(F), \mathbb{Z}[1 / 2]) .
$$

But from Lemma A.9, it also follows that the line $E_{p, 1}^{2, \text { alt }}$ is in fact trivial. Although the spectral sequence for the complex $C_{\bullet}^{\text {alt }}(F)$ does not degenerate at the $E^{2}$-term, its differentials are the maps $\mathcal{R} \mathcal{P}_{q}^{1}(F) \rightarrow \mathrm{H}_{q-1}(\mathrm{~N}(F), \mathbb{Z}[1 / 2])$, and all information is contained in the long exact sequence of Proposition A.10.

We see, using the comparison map $C_{\bullet}(F) \rightarrow C_{\bullet}^{\text {alt }}(F)$, that the differentials $d^{q-1}$ : $E_{0, q}^{q-1} \rightarrow E_{q-2,1}^{q-1}$ are surjective (with $\mathbb{Z}[1 / 2]$-coefficients). In the $E^{\infty}$-term, we then find $E_{q, 1}^{\infty} \cong E_{q, 1}^{\infty}$,alt $\cong 0$. Moreover, we can identify the $\mathcal{R} \mathcal{P}_{q}^{1}(F)$ groups as the entries 
$E_{0, q}^{q}$. The connecting map $\mathcal{R P}_{q}^{1}(F) \rightarrow \mathrm{H}_{q-1}(\mathrm{~N}(F), \mathbb{Z}[1 / 2])$ is the differential $d^{q}$ : $E_{0, q}^{q} \rightarrow E_{q-1,0}^{q}$. In particular, the groups $\mathcal{R} \mathcal{P}_{q}^{1}(F)$ can be identified (in the spectral sequence for $C_{\bullet}(F)$ ) with the kernel of the two differentials $d^{1}: E_{0, q}^{1} \rightarrow E_{0, q-1}^{1}$ and $d^{q-1}: E_{0, q}^{q-1} \rightarrow E_{q-2,1}^{q-1}$.

A.3. Low-dimensional computations. We note some further well-known statements on the (refined) scissors congruence groups, cf. Dup01 and Hut11b].

Proposition A.12. Let $F$ be an infinite field.

(1) $\mathcal{R P}_{q}^{1}(F)=0$ for $q \leq 1$.

(2) $\mathcal{P}_{q}^{1}(F)=0$ for $q \leq 2$.

(3) $\mathcal{R P}_{2}^{1}(F)=\mathrm{I}(F)$, where $I$ denotes the fundamental ideal in the Witt ring of $F$.

Proof. By definition $\mathcal{R} \mathcal{P}_{q}^{1}(F)$ is the $\mathrm{SL}_{2}(F)$-equivariant homology of the quotient $C_{\bullet}^{\text {alt }}(F) / F_{0} C_{\bullet}^{\text {alt }}(F)$. Since $\mathrm{SL}_{2}(F)$ acts transitively on the set of pairs of distinct points in $\mathbb{P}^{1}(F)$, the complex $C_{\bullet}^{\text {alt }}(F)$ has one copy of $\mathbb{Z}$ in degree 0 and one copy of $\mathbb{Z}$ in degree 1 . But these are both degenerate configurations, so they are contained in the subcomplex $F_{0} C_{\bullet}^{\text {alt }}(F)$. This proves the first claim (and part of the second claim).

(2) follows similarly, using that the action of $\mathrm{PGL}_{2}(F)$ on 3 distinct points in $\mathbb{P}^{1}(F)$ is also transitive. The result is a single copy of $\mathbb{Z}$ in degree 2 of the complex $\mathrm{PGL}_{2}(F) \backslash C_{\bullet}^{\text {alt }}(F)$, which is killed by the differential from degree 3 .

(3) is a reformulation of the computations in Hut11b, Section 4.5].

We want to note that the low-degree part of the long exact sequence from Proposition A.10 is the Bloch-Wigner sequence proved in Hut11b. In the notation of this appendix, it reads (all groups with $\mathbb{Z}[1 / 2]$-coefficients):

$$
0 \rightarrow \mathrm{H}_{3}(\mathrm{~N}(F)) \rightarrow \mathrm{H}_{3}\left(\mathrm{SL}_{2}(F)\right) \rightarrow \mathcal{R} \mathcal{P}_{3}^{1}(F) \rightarrow \mathrm{H}_{2}(\mathrm{~N}(F)) \rightarrow \mathrm{H}_{2}\left(\mathrm{SL}_{2}(F)\right) \rightarrow 0 .
$$

There would be a lot more to say about the refined scissors congruence groups. We only make two final remarks. The relation of $\mathrm{H}_{3}\left(\mathrm{SL}_{2}(\mathbb{C})\right)$ to actual scissors congruences in $S^{3}$ and $\mathbb{H}^{3}$ is the subject of the book Dup01. The FriedlanderMilnor conjecture is equivalent to unique divisibility of the scissors congruence groups $\mathcal{P}_{\bullet}^{1}(F)$. This is known so far only for $\mathcal{P}_{3}^{1}(F)$ by the work of Suslin, Dupont and Sah.

\section{REFERENCES}

[AB08] P. Abramenko and K.S. Brown. Buildings. Graduate Texts in Mathematics 248. Springer, 2008.

[Ati56] M.F. Atiyah. On the Krull-Schmidt theorem with applications to sheaves. Bull. Soc. Math. France 84 (1956), 307-317.

[Ati57] M.F. Atiyah. Complex analytic connections in fibre bundles. Trans. Amer. Math. Soc. 85 (1957), no. 1, 181-207.

[Bro94] K.S. Brown. Cohomology of groups. Corrected reprint of the 1982 original. Graduate Texts in Mathematics, 87. Springer, 1994.

[Dup01] J.L. Dupont. Scissors congruences, group homology and characteristic classes. Nankai Tracts in Mathematics 1. World Scientific, 2001.

[FM84] E.M. Friedlander and G. Mislin. Cohomology of classifying spaces of complex Lie groups and related discrete groups. Comment. Math. Helv. 59 (1984), 347-361.

[Har77] R. Hartshorne. Algebraic geometry. Graduate Texts in Mathematics 52. Springer, 1977.

[Hut11a] K. Hutchinson. A refined Bloch group and the third homology of $S L_{2}$ of a field. J. Pure Appl. Algebra 217 (2013), 2003-2035.

[Hut11b] K. Hutchinson. A Bloch-Wigner complex for $S L_{2}$. J. K-theory 12 (2013), no. 1, 15-68.

[Knu01] K.P. Knudson. Homology of linear groups. Progress in Mathematics, 193. Birkhuser Verlag, Basel, 2001. 
[Mis94] G. Mislin. Tate cohomology for arbitrary groups via satellites. Topology Appl. 56 (1994), no. 3, 293-300.

[Rah13] A.D. Rahm. The homological torsion of $\mathrm{PSL}_{2}$ of the imaginary quadratic integers. Trans. Amer. Math. Soc. 365 (2013), no. 3, 1603-1635.

[Rah14] A.D. Rahm. Accessing the cohomology of discrete groups above their virtual cohomological dimension. J. Alg. 404 (2014), no. C, 152-175.

[Ros73] M. Rosen. $S$-units and $S$-class group in algebraic function fields. J. Algebra 26 (1973), 98-108.

[Ser80] J.-P. Serre. Trees. Springer, 1980.

[Stu76] U. Stuhler. Zur Frage der endlichen Präsentierbarkeit gewisser arithmetischer Gruppen im Funktionenkörperfall. Math. Ann. 224 (1976), no. 3, 217-232.

[Stu80] U. Stuhler. Homological properties of certain arithmetic groups in the function field case. Invent. Math. 57 (1980), no. 3, 263-281.

[Sus90] A.A. Suslin. $K_{3}$ of a field and the Bloch group. Trudy Mat. Inst. Steklov., 183:190-199, 229, 1990. Translated in Proc. Steklov Inst. Math. 1991, no. 4, 217-239, Galois Theory, rings, algebraic groups and their applications (Russian)

Matthias Wendt, Fakultät Mathematik, Universität Duisburg-Essen, Thea-LeymannStrasse 9, 45127, Essen, Germany

E-mail address: matthias.wendt@uni-due.de 